6
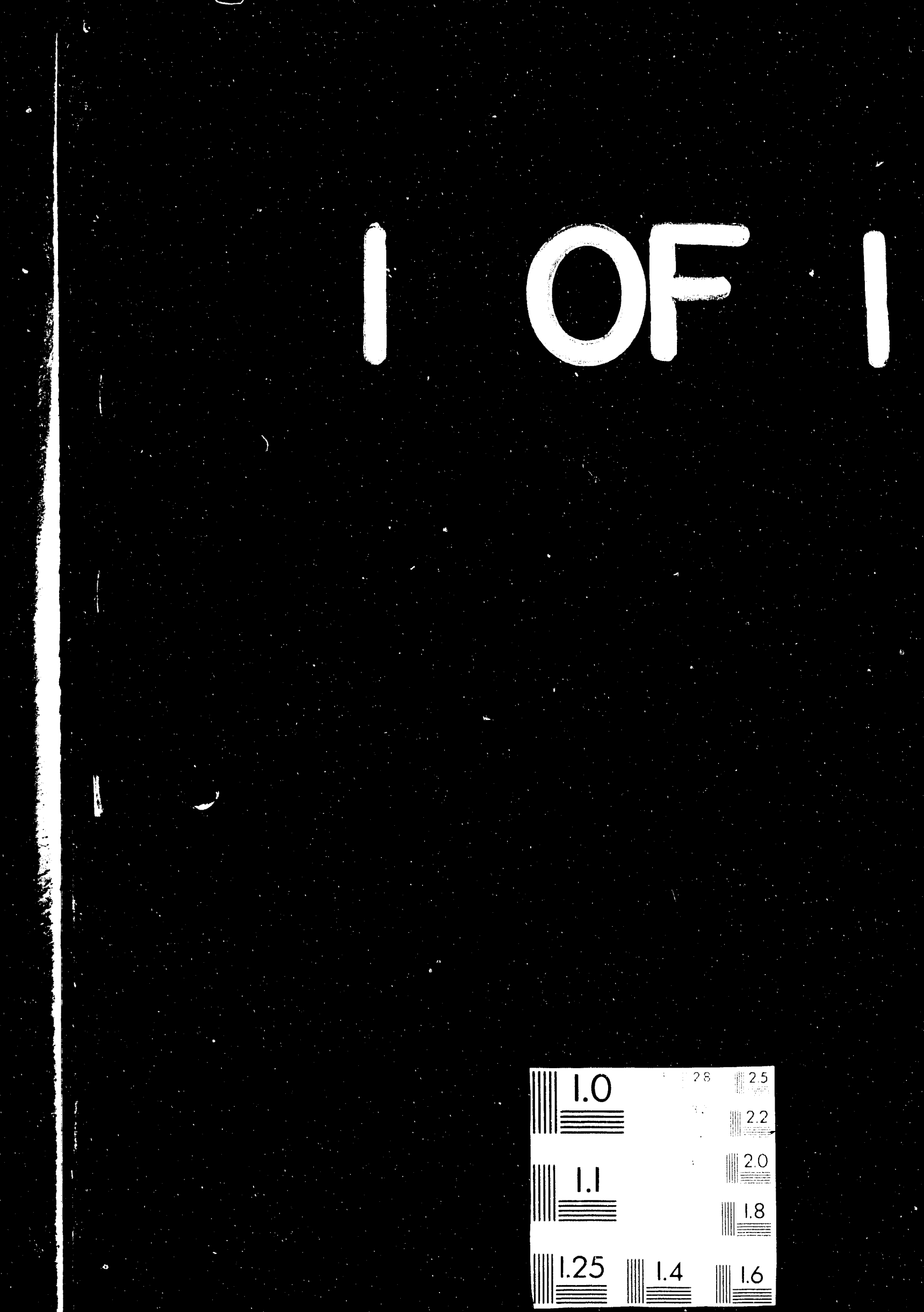
Instrumentation and Controls Division

\section{OTDR STRAIN GAUGE FOR SMART SKINS}

Stephen W. Kercel

Date Published-September 1993

Prepared by

OAK RIDGE NATIONAL LABORATORY

Oak Ridge, Tennessee 37831-6285 managed by

MARTIN MARIETTA ENERGY SYSTEMS, INC.

for the

U. S. DEPARTMENT OF ENERGY

under contract DE-AC05-84OR21400 


\section{CONTENTS}

LIST OF FIGURES $\ldots \ldots \ldots \ldots \ldots \ldots \ldots \ldots \ldots \ldots \ldots \ldots \ldots \ldots$

LIST OF TABLES $\ldots \ldots \ldots \ldots \ldots \ldots \ldots \ldots \ldots \ldots \ldots \ldots \ldots \ldots \ldots \ldots$

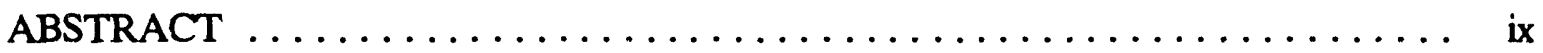

1. MEASURING STRAIN WITH OPTICAL FIBERS

EMBEDDED IN COMPOSITES $\ldots \ldots \ldots \ldots \ldots \ldots \ldots \ldots \ldots \ldots \ldots$

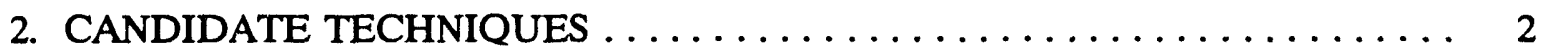

3. LIMITS ON OTDR PERFORMANCE $\ldots \ldots \ldots \ldots \ldots \ldots \ldots \ldots \ldots \ldots \ldots$

3.1 THEORETICAL BASIS . . . . . . . . . . .

3.2 PRACTICAL LIMITS ON PERFORMANCE $\ldots \ldots \ldots \ldots \ldots \ldots \ldots$

4. OTDR STRAIN GAUGE SETUP $\ldots \ldots \ldots \ldots \ldots \ldots \ldots \ldots \ldots \ldots \ldots$

5. EXPERIMENTAL RESULTS $\ldots \ldots \ldots \ldots \ldots \ldots \ldots \ldots \ldots \ldots \ldots \ldots \ldots$

6. OBSERVATION VS THEORY $\ldots \ldots \ldots \ldots \ldots \ldots \ldots \ldots \ldots \ldots \ldots \ldots \ldots$

7. AREAS OF FURTHER EXPLORATION $\ldots \ldots \ldots \ldots \ldots \ldots \ldots \ldots \ldots$

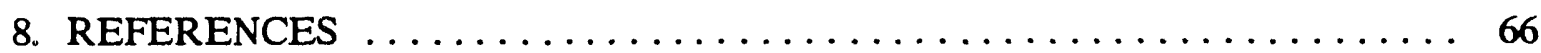




\section{LIST OF FIGURES}

Figure Page

1 Reflection-based $\mathrm{OTDR}$ layout $\ldots \ldots \ldots \ldots \ldots \ldots \ldots \ldots \ldots \ldots \ldots \ldots$

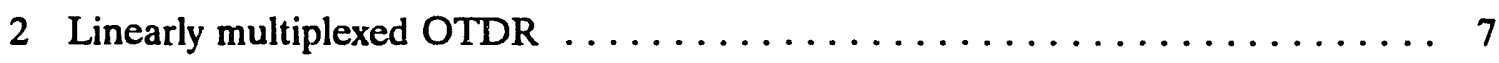

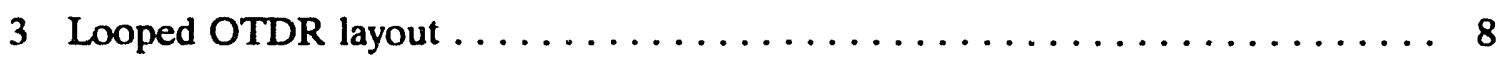

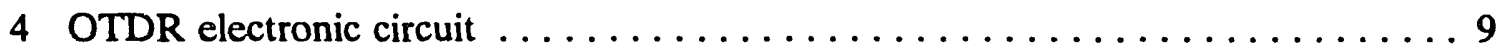

5 Mechanical support of gauge fiber $\ldots \ldots \ldots \ldots \ldots \ldots \ldots \ldots \ldots \ldots \ldots \ldots \ldots$

6 Overall OTDR strain gauge system $\ldots \ldots \ldots \ldots \ldots \ldots \ldots \ldots \ldots \ldots \ldots \ldots \ldots \ldots \ldots \ldots$

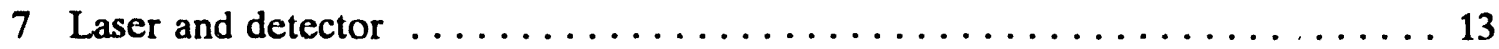

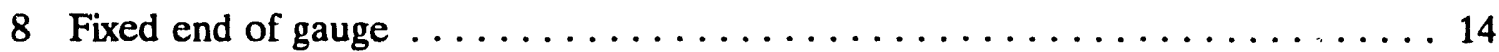

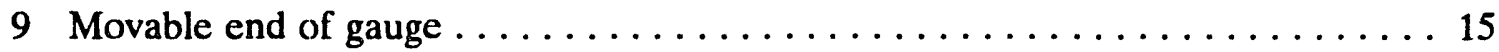

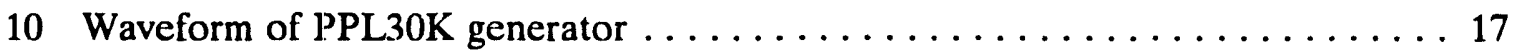

11 Waveform of OIG-502 generator $\ldots \ldots \ldots \ldots \ldots \ldots \ldots \ldots \ldots \ldots \ldots \ldots \ldots$

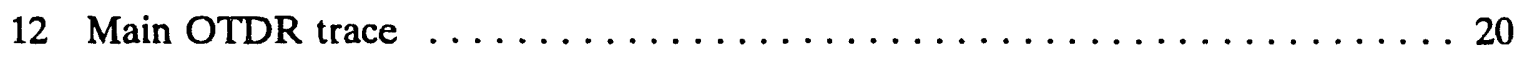

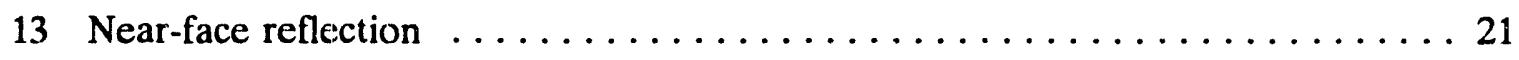

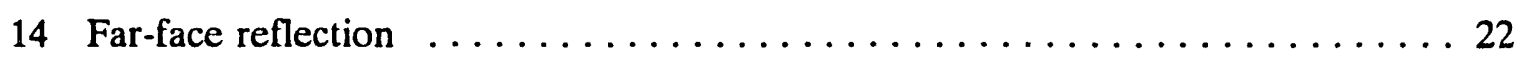

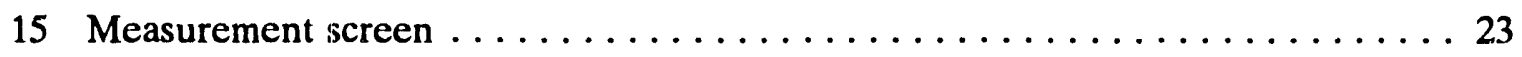

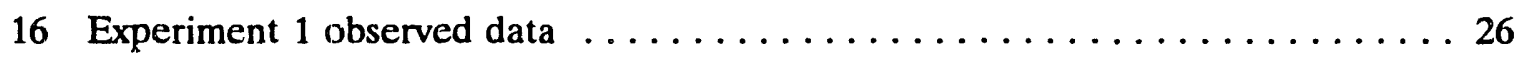

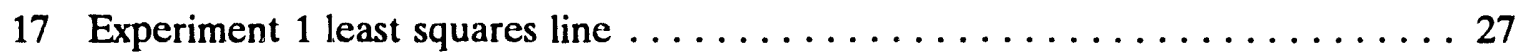

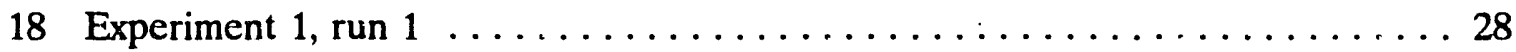

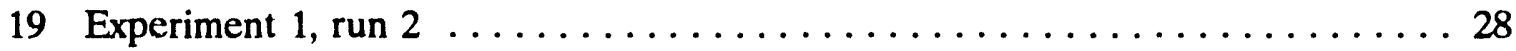

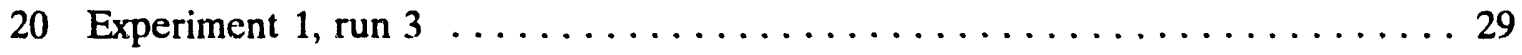

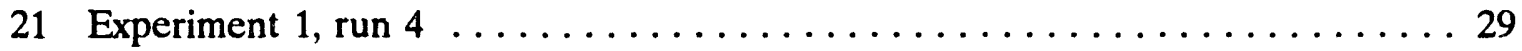

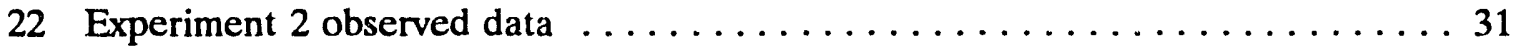

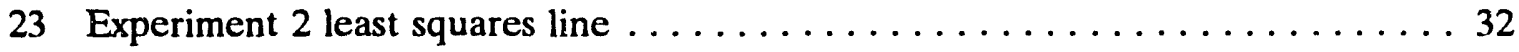

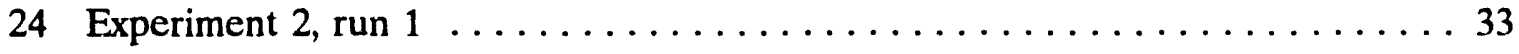

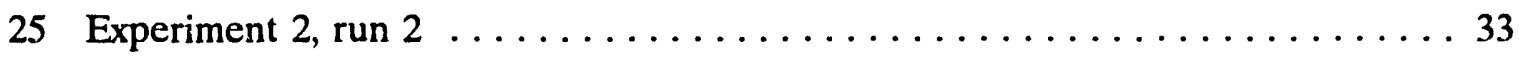

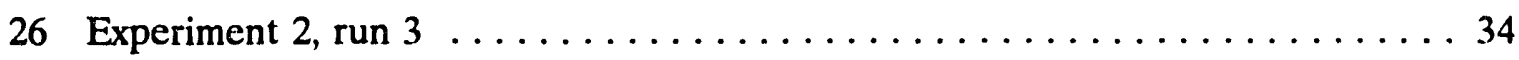

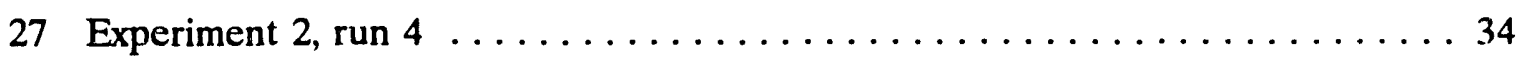

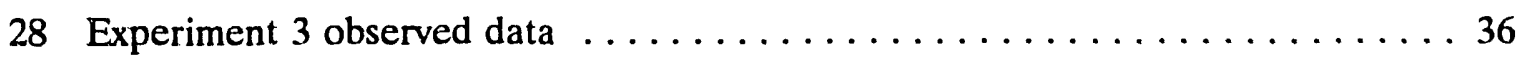

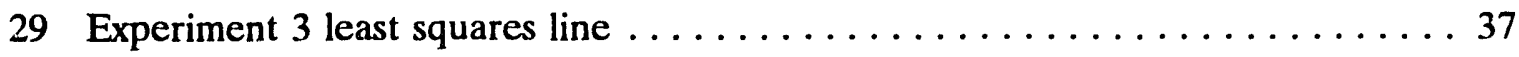

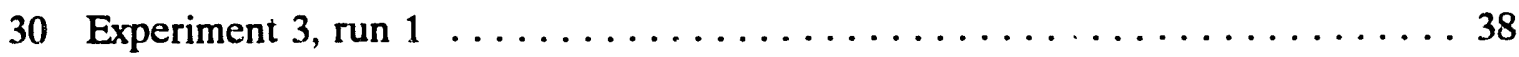




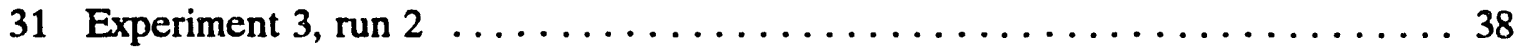

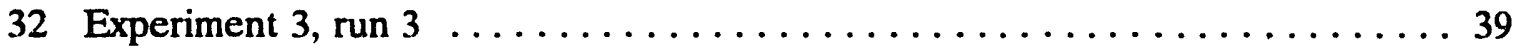

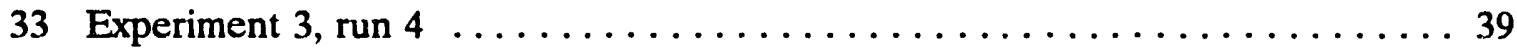

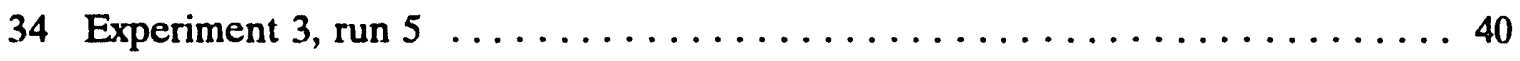

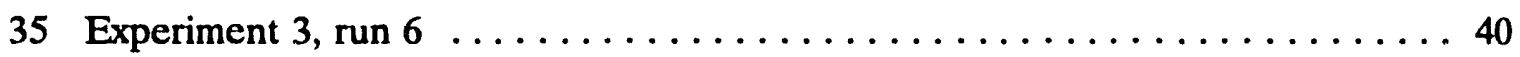

36 Experiment 4 observed and least squares $\ldots \ldots \ldots \ldots \ldots \ldots \ldots \ldots \ldots \ldots$

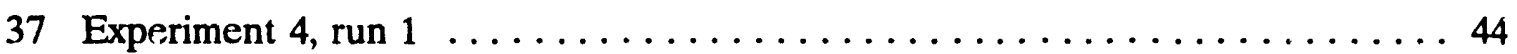

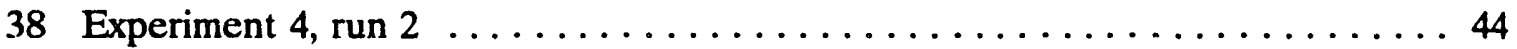

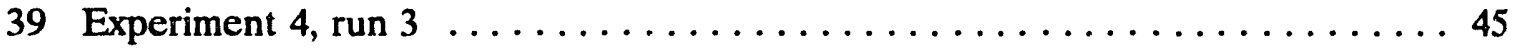

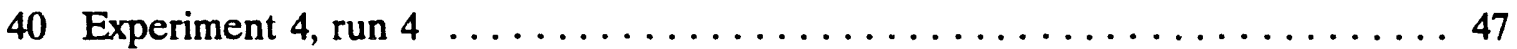

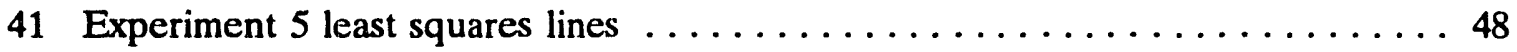

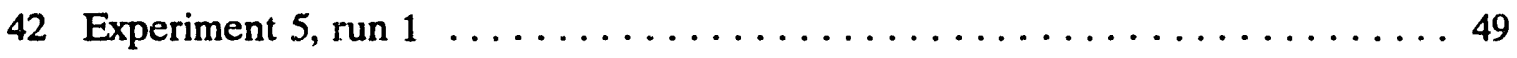

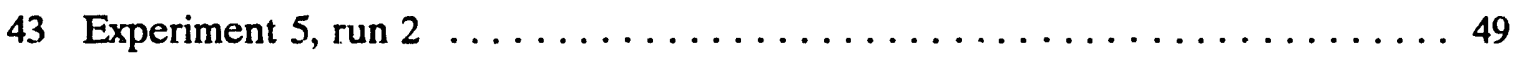

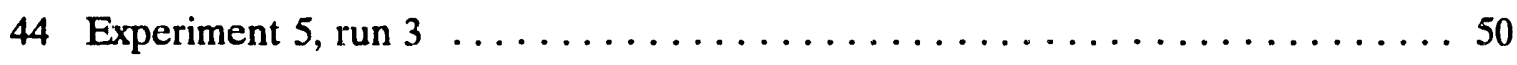

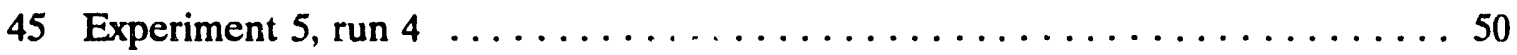

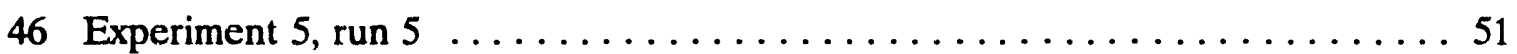

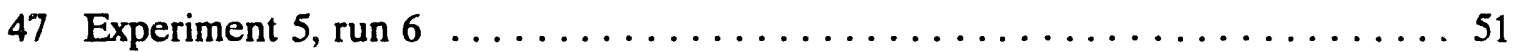

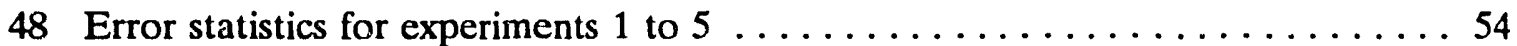

49 Error statistics for experiments 2 and $5 \ldots \ldots \ldots \ldots \ldots \ldots \ldots \ldots \ldots \ldots \ldots \ldots \ldots \ldots \ldots$

50 Standard deviation for several layouts $\ldots \ldots \ldots \ldots \ldots \ldots \ldots \ldots \ldots \ldots \ldots \ldots \ldots \ldots$

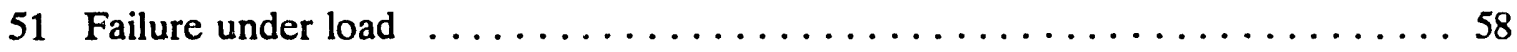

52 Time of flight vs loading-Opto-Electronics OFM $10 \ldots \ldots \ldots \ldots \ldots$

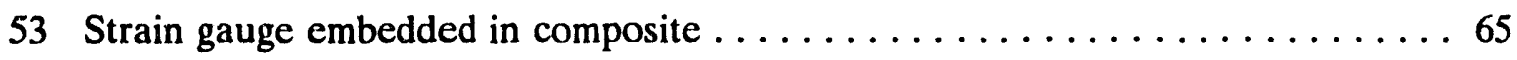




\section{LIST OF TABLES}

Table

Page

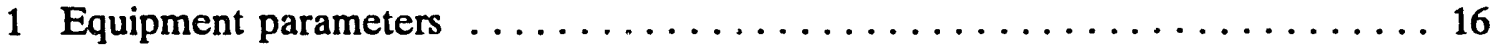

2 Repeatability of OTDR readings-comparison of Tektronix OIG-502

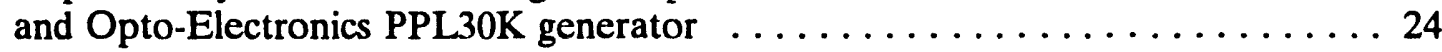

3 Experiment $1-$ Time of flight vs applied strain $\ldots \ldots \ldots \ldots \ldots \ldots \ldots \ldots$

4 Experiment $2-$ Time of flight vs applied strain $\ldots \ldots \ldots \ldots \ldots \ldots \ldots \ldots \ldots$

5 Experiment $3-$ Time of flight vs applied strain $\ldots \ldots \ldots \ldots \ldots \ldots \ldots \ldots$

6 Experiment 4 -Time of flight vs applied strain $\ldots \ldots \ldots \ldots \ldots \ldots \ldots \ldots \ldots$

7 Experiment 5 -Time of flight vs applied strain $\ldots \ldots \ldots \ldots \ldots \ldots \ldots$

8 Error statistics-strain applied $\ldots \ldots \ldots \ldots \ldots \ldots \ldots \ldots \ldots \ldots \ldots \ldots \ldots$

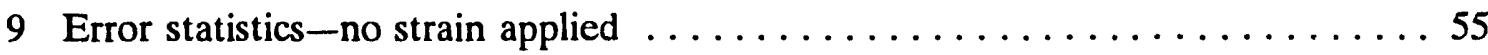

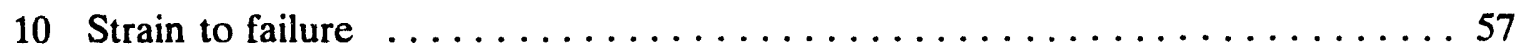

11 Time of flight measured with OFM 10 fiber monitor $\ldots \ldots \ldots \ldots \ldots$

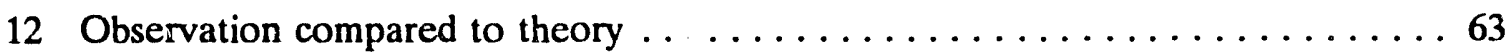




\begin{abstract}
Optical time-domain reflectometry (OTDR) is a simple and rugged technique for measuring quantities such as strain that affect the propagation of light in an optical fiber. For engineering applications of OTDR, it is important to know the repeatable limits of its performance. The author constructed an OTDR-based, submillimeter resolution strain measurement system from off-the-shelf components. The systems repeatably resolves changes in time of flight to within $\pm 2 \mathrm{ps}$. Using a $1-\mathrm{m}$, single-mode fiber as a gauge and observing the time of flight between Fresnel reflections, a repeatable sensitivity of 400 microstrains was observed. Using the same fiber to connect the legs of a 3-dB directional coupler to form a loop, a repeatable sensitivity of 200 microstrains was observed. Realizable changes to the system that should improve the repeatable sensitivity to 20 microstrains or less are discussed.
\end{abstract}




\section{MEASURING STRAIN WITH OPTICAL FIBERS EMBEDDED IN COMPOSITES}

Smart skins are composite materials with embedded sensors that detect specific parameters of the composite. The sensors are connected to electronic equipment that monitors the condition of the composite. Optical fibers are suitable sensor elements for smart skins. If properly embedded, they will not perturb the mechanical properties of the composite. The same fiber can simultaneously act as a sensing transducer to measure several different quantities and as a signal path to convey the sensed data to the monitoring electronics.

This research explores the feasibility of simultaneously measuring temperature and strain. It includes proof-of-principle experiments in optical time-domain reflectometry- (OTDR-) based strain measurements (initial experimental results reported here) and fluorescence-based temperature measurements (experimental results reported elsewhere). The concept uses a sensing fiber coated with fluorescent material and connected to near-infrared (ir) OTDR equipment via a directional coupler. Both near-ir and ultraviolet (uv) excitations are applied to the fiber. The visible fluorescence resulting from the uv excitation of the coating is observed, and a strain-independent observation of temperature is deduced from the fluorescence spectrum. Time of flight of the near-ir pulse through the gauge fiber is observed. From time-of-flight and temperature observations, a temperature-compensated value of strain is deduced.

If the sensing fiber is embedded in a composite, it should simultaneously sense the strain and temperature in the material. The issues of bonding the fiber to the composite matrix (ensuring that strain in the fiber is the same as the strain in the composite) and physical connection between the embedded sensing fiber and the outside world are not addressed by this research.

This report addresses proof-of-principle observations of OTDR fiber-optic strain gauges. The objective was to discover the practical limitations and capabilities of OTDR strain measurement to assess whether OTDR is potentially suitable for embedded strain measurement and to identify problems in implementation. Experiments involved the application of known amounts of strain to nonembedded fibers.

Repeatable strain sensitivity of 200 microstrains was observed with a $1-\mathrm{m}$ gauge fiber. Several changes in the experimental setup are expected to lead to sensitivity on the order of 10 microstrains with a $1-\mathrm{m}$ gauge fiber. These results will be published in the proceedings of the Society of Photo-Optical Instrumentation Engineers (SPIE) (Kercel 1992). 


\section{CANDIDATE TECHNIQUES}

The strain measurement technique being investigated for smart skins is OTDR (Zimmermann 1990a). The operative principle is that time of flight of a light pulse through a gauge fiber varies with strain. A major advantage of OTDR is that it gives an absolute reading; the measured value does not depend on event counting. No reference fiber is required. Perturbations in the lead-in fiber do not change the inäicated strain reading. OTDR is not difficult to align. The physical implementation is simple and rugged; an OTDR sensor fiber should survive the compositing process.

OTDR has several shortcomings. Notably, the real-time signal processing is relatively expensive. Other shortcomings are that the resolution, sensitivity, and dynamic range are not as good as with some other techniques. However, as our experimental data show, the performance of OTDR is better than generally appreciated. For many applications, the resolution, sensitivity, and dynamic range are sufficient. In such cases, giving away unneeded capability to get simplicity and ruggedness is a sensible trade-off.

Two straightforward ways of measuring strain in a gauge fiber are possible with OTDR equipment (Zimmermann 1990a). The reflective scheme uses the equipment in a conventional reflectometer configuration and observes the time of flight of a light pulse between discontinuities in a gauge fiber. The looping method uses the same equipment but is configured with the gauge fiber in a loop. The OTDR equipment observes the transit time of the pulse through many passes through the loop. The looping scheme has potentially much greater strain sensitivity than does the reflective scheme.

OTJR is relatively expensive when it is done with general-purpose, high-performance laboratory instrumentation. If OTDR proves to be satisfactory in all respects but cost, then one of the lines of future research that could be undertaken would be to develop specific-purpose signal processing electronics that would be relatively inexpensive to manufacture in quantity.

Another strain measurement technique is fiber cross talk (Dunphy 1982). The operative principle is that the relative amounts of the energy in the two cross-talk-coupled cores at any given measuring point varies with strain. A major advantage of cross talk is that it can be designed to provide a temperature-independent indication of strain. A major disadvantage is that alignment is difficult. The sensing fiber uses two cores. All the excitation energy must be coupled into one core, with none going to the other. At the output end, the outputs of the two cores must be measured independently.

Several different strain measurement techniques are based on interferometry (Udd 1991), including the SAGNAC, Mach-Zehnder, and Michelson techniques. The operative principle is that the amount that the signal is phase shifted in a strained fiber depends on the strain and that the phase shift can be deduced by observing the interference between the outputs of the strained fiber and an unstrained fiber. The major problems with interferometric techniques is that the reference signal must be isolated from the environment and that perturbations in the lead-in fibers can affect the indicated strain reading. Another drawback is that starting at zero strain, the number of times that the phase shifts through $2 \pi$ radians must be tracked to provide the strain reading. Interferometry is a laboratory technique that does not appear readily adaptable to field use.

Another strain measurement technique is polarimetry (Waite 1988). The operative principle is that the state of polarization of a signal in a birefringent fiber changes continuously with position down the fiber. Correspondingly, at a given observation point, the state of polarization changes with strain. In a manner similar to interferometry, starting at 
zero strain, the number of rotations of the state of polarization must be tracked to provide the strain reading. It is easy to lose count. Reproducible polarization must be coupled into the input end, and the state of polarization must be determined $\therefore$ the output end. Trying to do this embedded in composites looks difficult.

Another strain measurement technique is microbending (Marvin 1984). The operative principle is that the attenuation at a microbend of a multimode fiber increases as the radius of the microbend becomes smaller. Conventional microbending strain-measuring techniques depend on the free movement of the microbend and do not lend themselves to embedding.

Another strain measurement technique is modal power distribution (Murphy 1990). The operative principle is that the power distribution in a few-mode fiber varies as a function of strain. This area appears to be promising for further exploration. The most serious apparent problem is that it requires the use of elliptical core fibers. 


\section{LIMITS ON OTDR PERFORMANCE}

\subsection{THEORETICAL BASIS}

The idea of using OTDR as a strain measurement technique is based on the proposition that the change in time of flight of an optical pulse through a gauge fiber depends in a predictable way on the strain in the fiber (Brininstool 1987). Given a fiber of length of $L$, effective index of refraction of $N$, and the speed of light in vacuum of $c$, the time of flight between the discontinuities is $\tau$,

$$
\tau=\frac{N L}{c}
$$

Note that both the length $L$ and the index of refraction $N$ are dependent on the tensile stress $\sigma$. Thus, the change in the time of flight with respec ${ }^{+}$to stress is given by applying the product differentiation rule.

$$
\frac{d \tau}{d \sigma}=\frac{1}{c}\left(N \frac{d L}{d \sigma}+L \frac{d N}{d \sigma}\right) .
$$

The first term describes the increase in the time of flight due to the elongation of the fiber under tension. The second term describes the decrease in the time of flight due to the decrease in index of refraction. (Note: A fiber under tension does not decrease in radius; it becomes less dense as it elongates.) Both effects have similar magnitude. Application of the chain rule and some algebraic manipulation lead to a relationship between the change in time of flight through the gauge fiber $\Delta \tau$ and the elongation of the gauge fiber $\Delta L$,

$$
\frac{\Delta \tau}{\Delta L}=\frac{N}{c}\left(1+\frac{L}{N} \frac{d N}{d L}\right]
$$

This equation can be solved for the elongation $\Delta L$ if the rate of change of the index of refraction is known. This rate can be determined from the properties of the fiber material and the equation

$$
\frac{d N}{d L}=-\left(\frac{N^{3}}{2 L}\right)\left[\rho_{12}-\nu\left(\rho_{11}+\rho_{12}\right)\right] .
$$

In this equation, $\rho_{i j}$ are strain optic tensor components, and $\nu$ is Poisson's ratio. $L$ is the gauge length; it can be measured directly. If the material properties are available from the fiber vendor, then $d N / d L$ can be computed directly. Once this property is known, the quantity $\Delta \tau / \Delta L$ can be computed. If the material properties are not available, then $\Delta \tau / \Delta L$ can be observed experimentally.

The average strain $\epsilon$ as a function of the observed time of flight $\Delta \tau$ between two discontinuities separated by a distance $L$ is computed by 


$$
\epsilon=\frac{\Delta L}{L}=\left[\frac{1}{L}\right]\left(\frac{\Delta L}{\Delta \tau}\right) \Delta \tau .
$$

Note that in OTDR, the observed time of flight, $\Delta \tau$, is found from $\Delta t_{1}-\Delta t_{0}$, the time delay between the peaks on the oscilloscope trace; and $K$, the number of times the light pulse passes through the gauge fiber. In reflective OTDR, $K=2$. In looping OTDR, $K$ is the number of peaks in the trace minus 1 ,

$$
\Delta \tau=\frac{\Delta t_{1}-\Delta t_{0}}{K} .
$$

The tiber photoelastic coefficient is $a$ (Zimmermann 1990a). For low-dispersion fiber (single mode of graded index multimode) $a$ remains constant as the pulse propagates down the fiber (Zimmermann 1990b). Starting with Zimmermann's definition of $a$, after a bit of manipulation, the following can be shown:

$$
\begin{gathered}
a=\frac{L}{N} \frac{d N}{d L} \\
\frac{\Delta \tau}{\Delta L}=\frac{N}{c}(1+a) \\
\epsilon=\left[\frac{1}{L}\right] \frac{c}{N} \frac{1}{1+a} \Delta \tau \\
\epsilon=\frac{\Delta t_{1}-\Delta t_{0}}{K L \frac{N}{c}}\left[\frac{1}{1+a}\right],
\end{gathered}
$$

where

$\epsilon=$ strain

$\Delta t_{1}-\Delta t_{0}=$ time between OTDR peaks displayed on scope,

$L=$ gauge length,

$c=$ speed of light in free space,

$N=$ fibcr index of refraction (sample can be measured),

$a=$ fiber photoelastic coefficient (sample can be measured),

$K=$ the number of times the pulse passes through the gauge.

\subsection{PRACTICAL LIMITS ON PERFORMANCE}

Two OTDR techniques are widely used (Garside 1988). A technique for performing general investigation and diagnosis of fibers observes the portion of the fiber Rayleigh 
scattering confined as backward traveling modes (Jackson 1984). To obtain high sensitivity and eliminate dead spots after discontinuities, the technique often uses detection based on photon counting. Another technique suitable for strain gauge applications, assuming the setup leads to reasonably sharp peaks in the oscilloscope trace, uses digital sampling (a technique for analog detection); in reflective OTDR this technique uses Fresnel reflections. The experiments described in this report are based on the observation of sharp peaks rather than Rayleigh scattering. Limitations on the performance of the system are imposed by the resolution and the dynamic range of the experimental apparatus.

Resolution of an OTDR setup can have two different meanings. Spatial resolution is the capability of the system to discern the length of the gauge fiber. Spatial precision is the capability to determine a small change in the length of the gauge fiber. Strain measurement deals with gauge lengths much greater than the limit imposed by spatial resolution but makes a precise observation of the elongation of the fiber as strain is applied. Therefore, it is the spatial precision, the minimum change in length detectable by the OTDR setup, that limits the performance of a strain gauge.

Spatial precision depends on the pulse width and signal-to-noise ratio (SNR) of the pulse as seen by the receiving electronics. The relationship is plotted in Fig. 4 of Garside's paper. For example, if the received pulse width is 100 ps and the SNR is 10 , then the uncertainty in spatial precision is <250 $\mu \mathrm{m}$ (Garside 1988).

Strain gauge sensitivity is the minimum discernible strain or the spatial precision divided by the gauge length. A strain gauge with a spatial precision of $100 \mu \mathrm{m}$ and a gauge length of $1 \mathrm{~m}$ (a million micrometers) has a sensitivity of 100 microstrains.

The dynamic range in decibels is the difference between the highest and lowest power levels in decibels with respect to a milliwatt available to and usable by the detector. It is affected by the losses in the fiber-optic system. The high end of the dynamic range depends on the maximum power available from the optical pulse generator and the maximum power at which the optical-to-electrical convertor will operate linearly. The low end of the dynamic range depends on the noise equivalent power of the detector and the number of observations averaged to make a measurement.

For a reflective setup (Fig. 1), the high end of the dynamic range is determined by assuming that the directional coupler is terminated in a perfectly cleaved end with no strain gauge fibers attached. This determination leads to the maximum possible reflection into the detector. Take the peak pulse power of the generator in decibels with respect to a milliwatt. Subtract the generator-to-pigtail coupling loss in decibels. Subtract the loss in decibels of the connector between the pigtail and the directional coupler. Subtract the insertion losses of the directional coupler in decibels for both directions. Subtract $14 \mathrm{~dB}$ due to the Fresnel reflection at the end of the directional coupler. Subtract the coupling loss of the connection of the directional coupler to the optical-to-electrical convertor. What remains is the maximum power seen by the detector. The high end of the dynamic range is the lesser of this maximum power seen by the detector or the maximum power for which the detector stays linear.

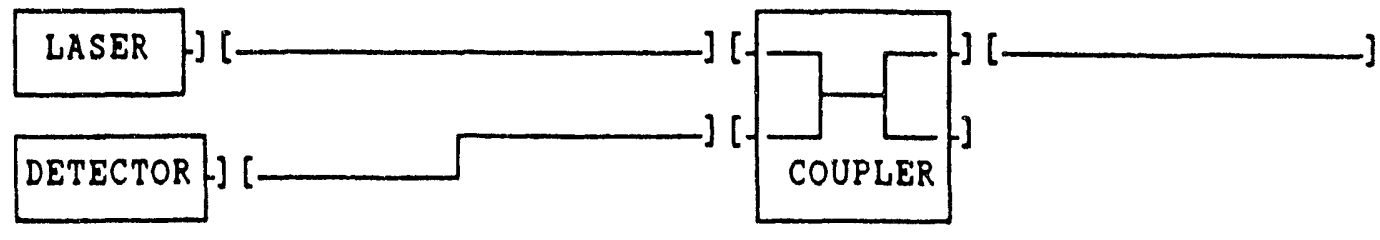

Fig. 1. Reflection-based OTDR layout. 
For a reflective setup, the low end of the dynamic range is the minimum signal to the detector that provides the SNR required by the resolution of the system. Assuming that the noise in the detector is uncorrelated for different observations, the noise floor can be lowered by averaging repeated observations. As compared to a single observation, the minimum discernible signal is inversely proportional to the square root of the number of observations in the average. Thus, a trade-off exists between dynamic range and observation time.

For a reflective setup, the dynamic range limits the number of observations of localized strain that can be observed simultaneously by a single setup. An OTDR setup can be connected to a long fiber divided into a sequence of segments (Fig. 2). The OTDR trace includes a peak for each discontinuity between segments. The average strain in each segment can be computed from the time of flight of the pulse between the discontinuities that bound the segment. The power reflected from a discontinuity is no stronger than $14 \mathrm{~dB}$ below the incident signal (Fresnel reflection for a perfectly cleaved end face) and may be $>40 \mathrm{~dB}$ down. In addition, each discontinuity has an insertion loss for the forward signal passing through it. If the discontinuity is well made, the insertion loss is on the order of $1 \mathrm{~dB}$. What limits the number of segments that can be observed at once is that the signal reflected from the last discontinuity must be sufficiently strong at the detector to provide the SNR needed to meet resolution requirements.
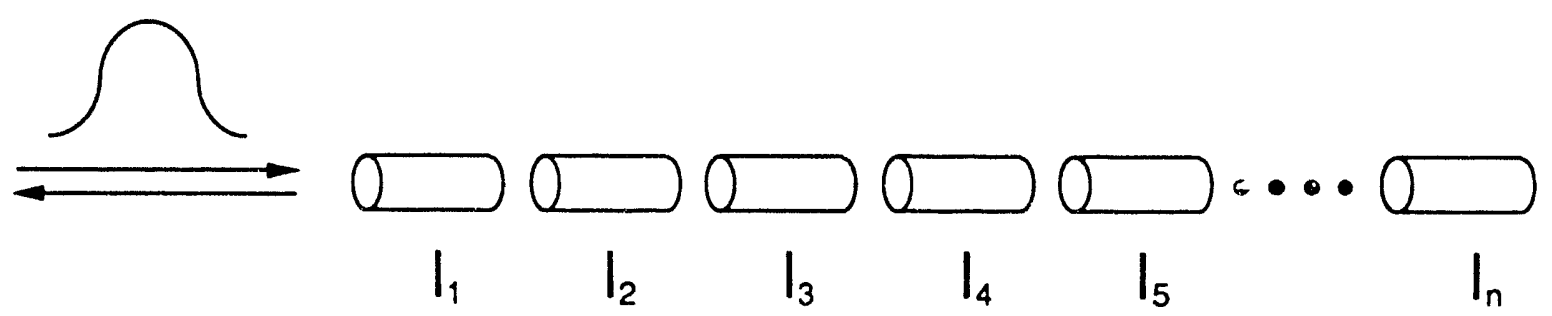

Fig. 2 Linearly multiplexed OTDR.

Given the dynamic range of an OTDR setup, determined as described above, assuming the same reflection factor and insertion loss for each discontinuity and assuming that all power ratios are expressed in decibels, the limiting number of discontinuities that can divide the fiber is found as follows: Starting with the dynamic range, add $14 \mathrm{~dB}$; then subtract two times the insertion loss of the connector that connects the directional coupler to the strain gauge. From this result, subtract the reflection factor of the discontinuity and then subtract two times the attenuation of the fiber that makes up the strain gauge. Divide the result by two times the insertion loss of the discontinuity. The greatest integer in this quotient is the number of segments allowed by the dynamic range of the system.

For a loop setup (Fig. 3), the high end of the dynamic range is determined by considering how much of the initial pulse of the train finds its way to the detector. Take the peak pulse power of the generator in decibels with respect to a milliwatt. Subtract the generator-topigtail coupling loss in decibels. Subtract the loss, in decibels, of the connector between the pigtail and the directional coupier. Subtract the insertion losses of the directional coupler in decibels for one direction. Subtract the detector-to-pigtail coupling loss in decibels. Subtract the loss in decibels of the connector between the pigtail and the directional coupler. What remains is the maximum power seen by the detector. The high end of the dynamic range is the lesser of this maximum power seen by the detector and the maximum power for which the detector stays linear. 


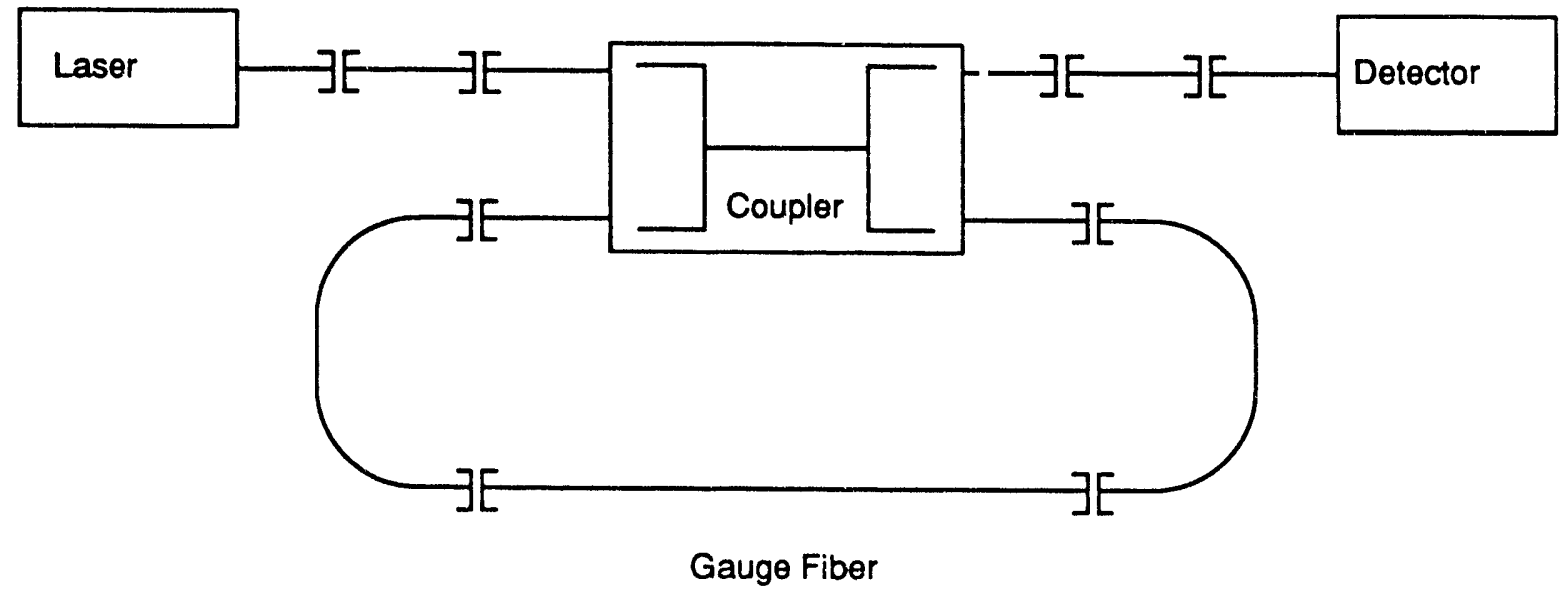

Fig. 3. Looped OTDR layout.

For a loop setup, the low end of the dynamic range is the minimum signal to the detector that provides the SNR required by the resolution of the system. On each successive pass of the pulse around the loop, the same fraction of its remaining power will be coupled into the leg leading to the detector. Eventually, the remaining power will fall below the noise floor of the detector.

In the loop setup, the dynamic range imposes a fundamental limit on the strain sensitivity of the gauge. The difference between the highest and lowest usable pulses limits the number of pulses in the train that can be observed and thus the number of passes that the pulse makes through the gauge fiber. The accuracy of the gauge depends on the number of passes observed.

One other major limit on strain sensitivity is imposed by the OTDR electronics (Fig. 4). At the start of the sampling cycle, a pulse from the trigger circuit fires the laser and also causes the detector, after a precise delay, to take one sample from the received event. A successive pulse from the trigger fires the laser again and causes the detector to take a sample from the next received event, using a delay slightly longer than that used for the preceding sample. After many such trigger pulses, the detector will have collected enough samples to constrict a replica of a typical received signal. Thus, a trace consisting of 512 samples is really a composite, made up of one sample each, from 512 consecutive events incident on the detector.

How accurately the resulting trace represents the many events from which it is drawn is limited by trigger jitter. If the timing between all trigger pulses were exactly the same, then the oscilloscope trace would be a perfect replica of the events that made it up. However, some variation, or jitter, occurs in the timing between trigger pulses. Jitter leads to uncertainty in the timing between features in the trace. In practical effect, jitter is seen as a drift in the time between peaks as the trace is displayed over several seconds. 


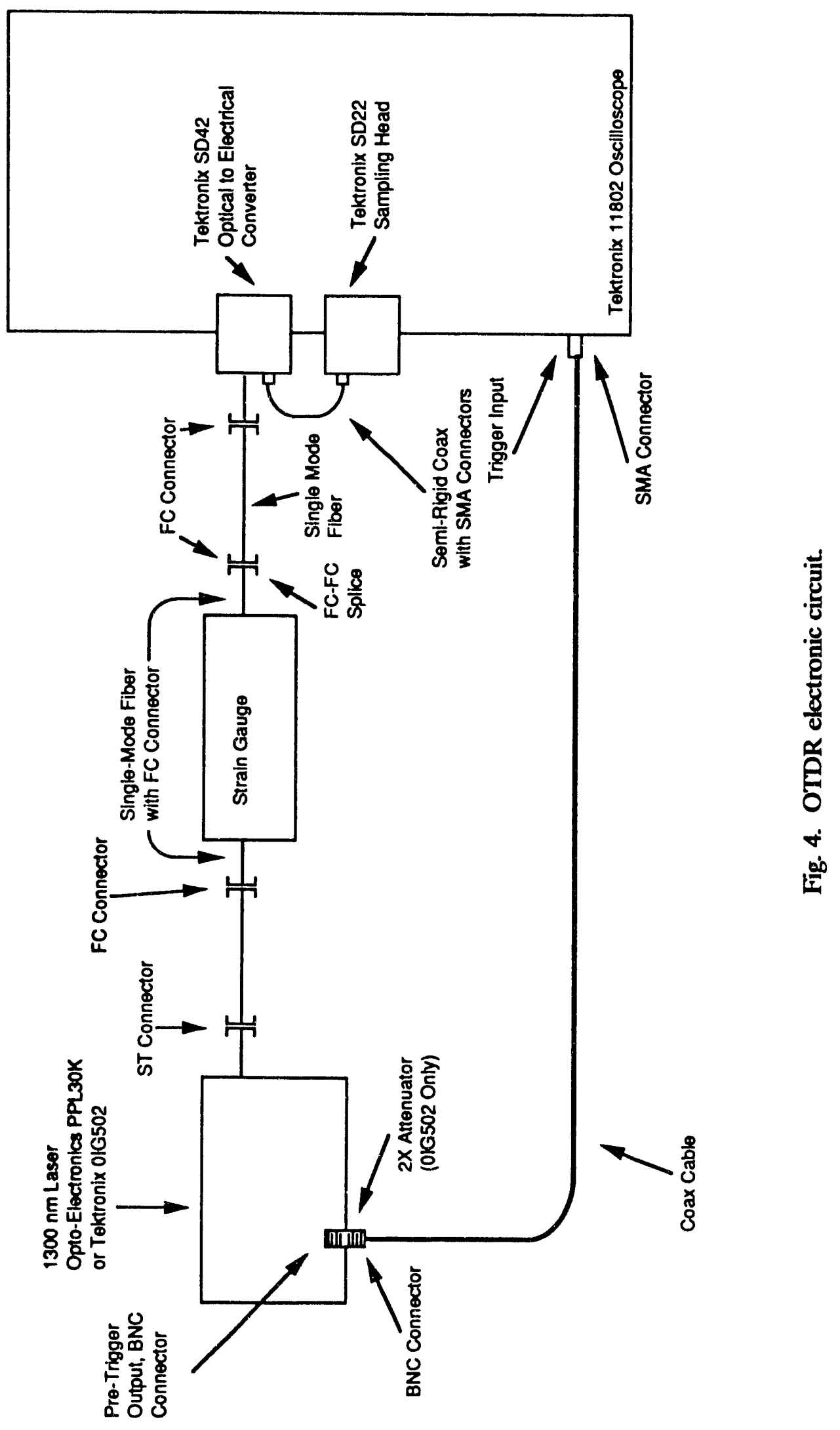




\section{OTDR STRAIN GAUGE SETUP}

The experimental setup is shown in Figs. 1 and 3 to 9. Figure 1 shows the optical setup for reflection-based OTDR. Figure 3 shows the optical setup for looped OTDR. Figure 4 shows the electronic circuit used with all strain gauge configurations. Figure 5 shows the mechanical setup used to support and apply a known amount of strain to the gauge fiber. Figure 6 is a photograph of the entire system. Figure 7 is a photograph of the laser and the detection system. Figure 8 shows the rigid support at the fixed end of the gauge fiber. Figure 9 shows the movable end of the gauge fiber.

In the reflection-based scheme (Fig. 1), excitation is provided by a pulsed laser. The excitation pulse passes through a directional coupler into the gauge fiber. As the pulse passes down the fiber, at each discontinuity a portion of its energy is reflected and propagates back toward the directional coupler. The reflected pulses are coupled by the directional coupler to the receiving electronics.

From the point of view of the receiving electronics, the signal is seen as a train of pulses, one from each discontinuity. The time delay between the pulses is the time of flight of the light pulse between the discontinuities. As strain is applied to the portion of the fiber between the discontinuities, the time of flight is increased as a predictable function of strain. Thus, by observing the change in the time delay in the received pulses, it is possible to calculate the strain experienced by the fiber between the discontinuities.

In the looping scheme (Fig. 3), excitation is provided by a pulsed laser. The excitation pulse passes through a directional or tap-off coupler. Part of the energy in the pulse passes to the leg connected to the detector. Part of the energy in the pulse passes to the leg connected to the gauge fiber. The other end of the gauge is connected to the remaining leg of the coupler to close the loop. When the pulse passing through the gauge reenters the coupler, part of it is coupled to the detector leg, and part is coupled back into the gauge for another pass. This process is repeated many times.

From the point of view of the receiving electronics, the signal is seen as a train of pulses, one from the initial pulse from the generator and one from each pass through the loop. The time delay between the pulses is the time of flight for the light pulse to make one circuit around the loop. As strain is applied to a portion of the fiber in the loop, the time of flight is increased as a predictable function of strain. Thus, by observing the change in the time between received pulses, it is possible to calculate the strain experienced by the fiber in the loop.

Figures 4 and 7 show the electronic setup used to generate the pulse and to process the received pulse train. Parameters are listed in Table 1 . Figures 10 and 11 show the waveforms of the generators. In each case, the trace was taken by connecting the generator to the detector with a $1-\mathrm{m}$ fiber.

The coupler is an Amphenol 50-50 fiber-optic directional coupler. The system operates at $1300 \mathrm{~nm}$ and uses single-mode, $9-\mu \mathrm{m}$ core silica-silica fibers.

The limit on strain sensitivity of this system is imposed by trigger jitter. The setup has a trigger jitter of \pm 2 ps. The noise-limited resolution can be obtained from Fig. 4 in Garside's paper (1988). For this setup, the received pulse width is less than 60 ps. The SNR is well above 20 for the weakest observed signal. Thus, if there were no time jitter, it would be possible to observe changes in time of flight of considerably less than 1 ps. Since the jitter limit is much more severe than the noise limit, the sensitivity and accuracy are dictated by jitter. That is, the actual time between any two peaks observed in a trace has an uncertainty of \pm 2 ps. 


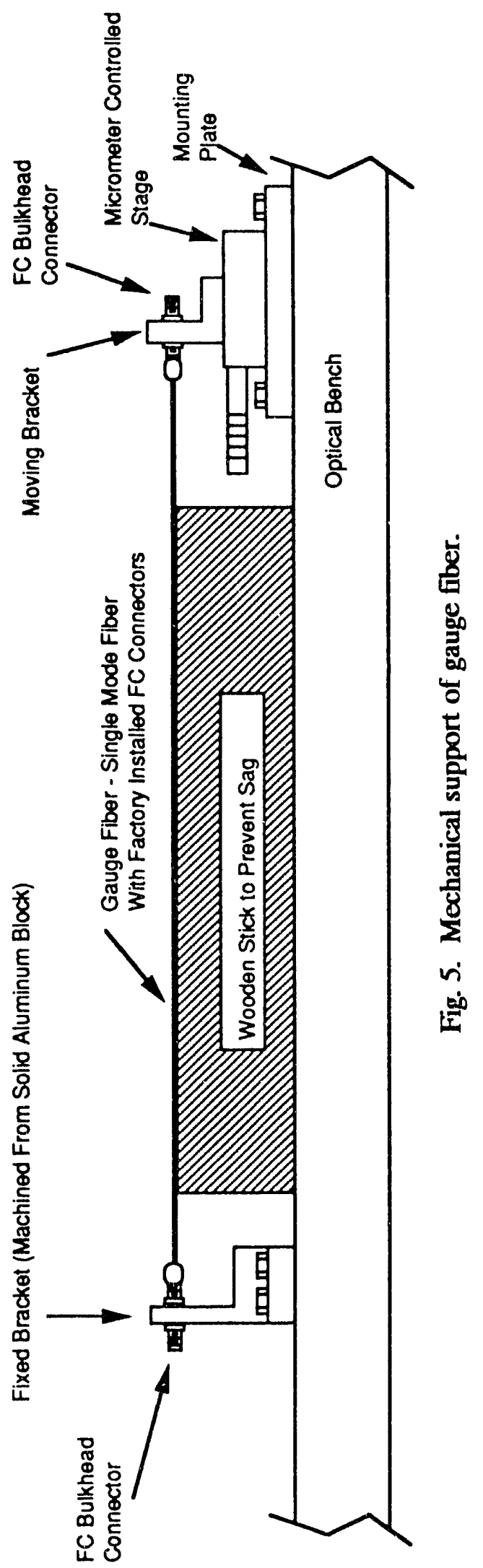




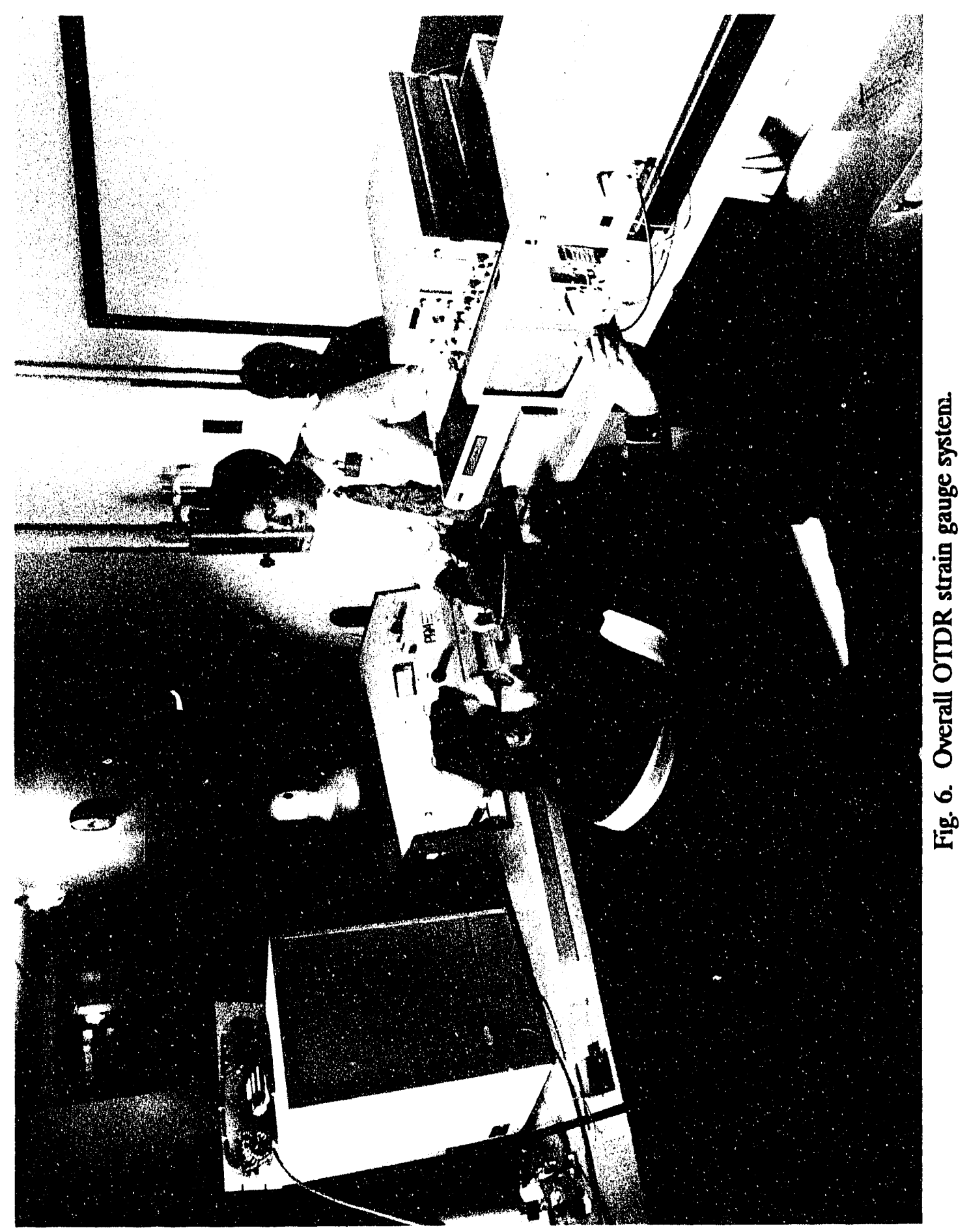




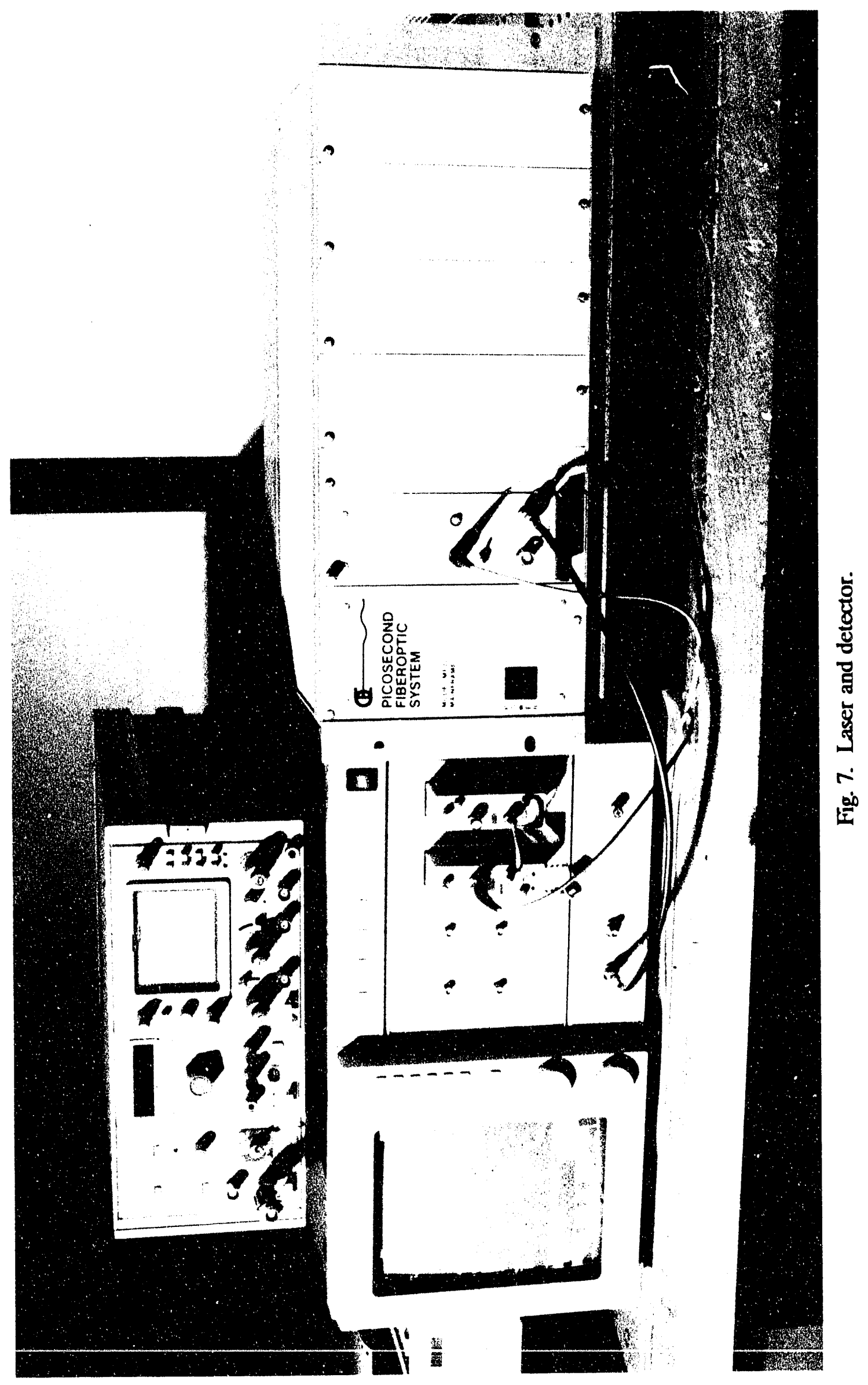




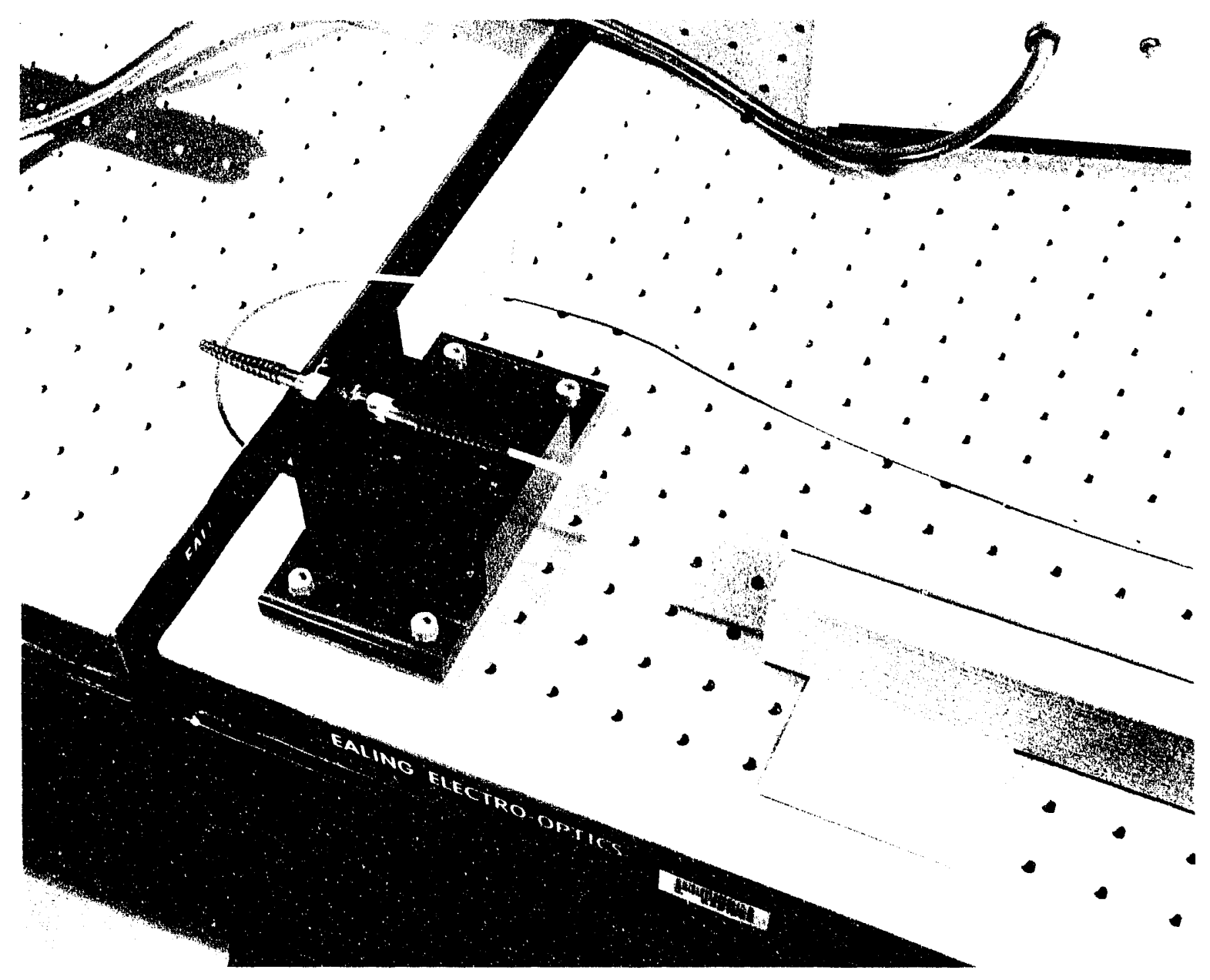

Fig. 8. Fixed end of gauge.

The mechanical setup for the OTDR strain gauge principle is shown schematically in Fig. 5. A gauge fiber with factory-installed FC connectors is mounted in rigidly supported FC bulkhead connectors. One end is fixed, as shown in Fig. 8. The support shown in Fig. 8 was machined from a single piece of metal to assure maximum rigidity. The other end of the gauge fiber is mounted to a manual micrometer-controlled translating stage, as shown in Fig. 9. The stage is supported on a large flat plate. The bulkhead mounted on the stage is machined such that the two bulkhead connectors supporting the gauge fiber are exactly the same height above the optical bench. Mechanical design of the system is intended to minimize deformation of the supports and slippage of the components. The gauge fiber is continuously supported to eliminate contribution to fiber tension from catenary effects.

At the zero offset position of the stage, the fiber is stretched taut. Strain is imposed by adjusting the micrometer to translate the stage so as to stretch the fiber. The amount of strain can be observed by reading the amount of stretch from the micrometer and dividing by the known gauge length. The resolution of the stage micrometer is $10 \mu \mathrm{m}$. 


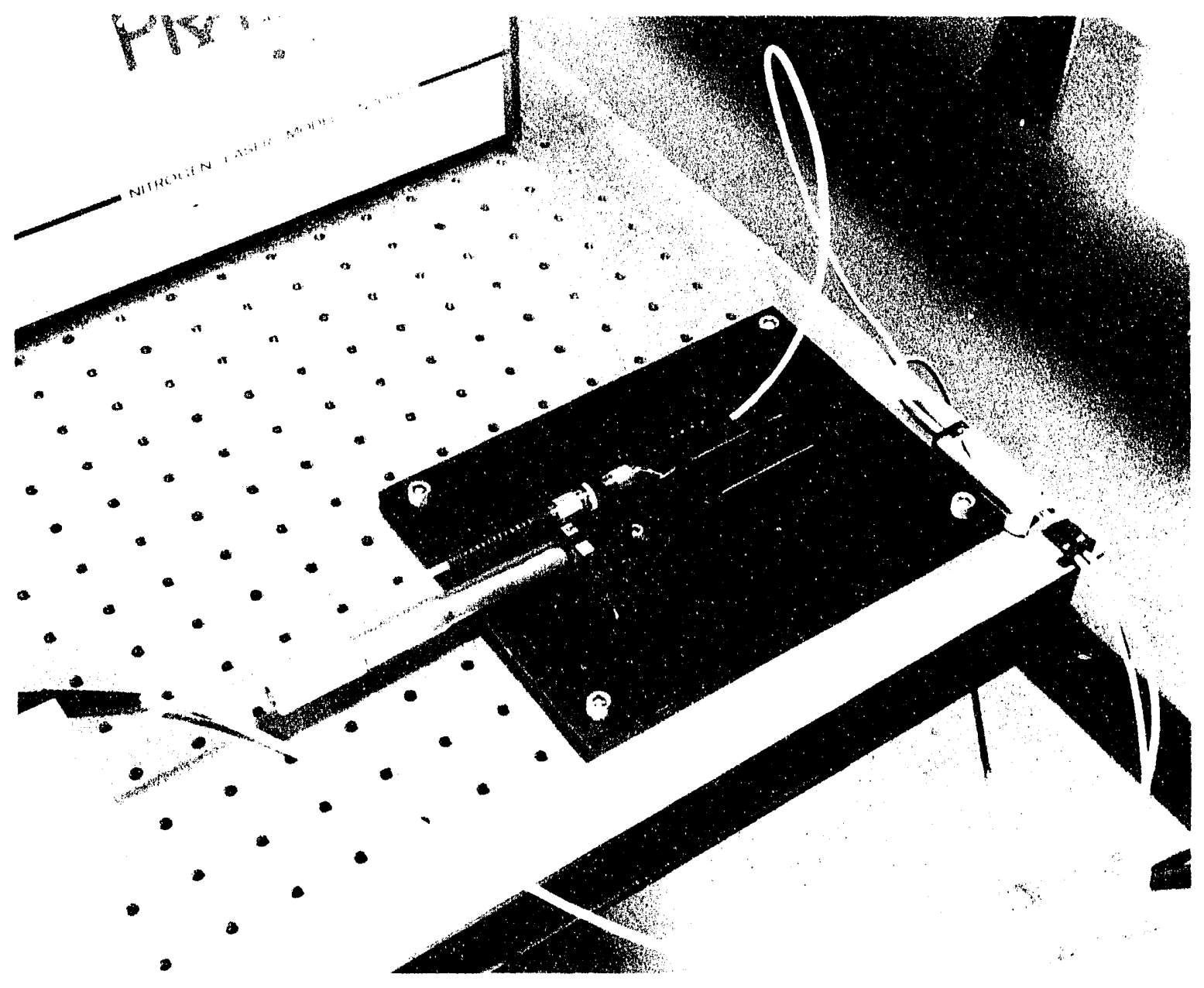

Fig. 9. Movable end of gauge. 
Table 1. Equipment parameters

\section{Receiving}

SD-42 bandwidth: $6.4 \mathrm{GHz}$

SD-42 impulse response: 55 ps (FWHM) ${ }^{a}$

SD-42 noise-equivalent power: $10 \mu \mathrm{W}$ (with SD-22)

SD-22 bandwidth: $12.5 \mathrm{GHz}$

Transmitting

Repetition rates

OIG-502: $10 \mathrm{kHz}$ (higher rates are less accurate)

PPL30K: $30 \mathrm{kHz}$ (fixed by manufacturer)

(The following properties were observed with the SD-42/SD-22/11802. The generator was connected to the SD-42 input via a single-mode 1-m-long silica-silica fiber.)

Peak power (displayed on scope)

OIG-502: $7.5 \mathrm{~mW}$

PPL30K: $19 \mathrm{~mW}$

Received FWHM pulse width (displayed on scope)

OIG-502: $57.6 \mathrm{ps}$

PPL30K: $57.2 \mathrm{ps}$

See Figs. 10 and 11 for traces of the PPL30K and the OIG-502 respectively.

${ }^{a} \mathrm{FWHM}=$ full-width half-maximum. 


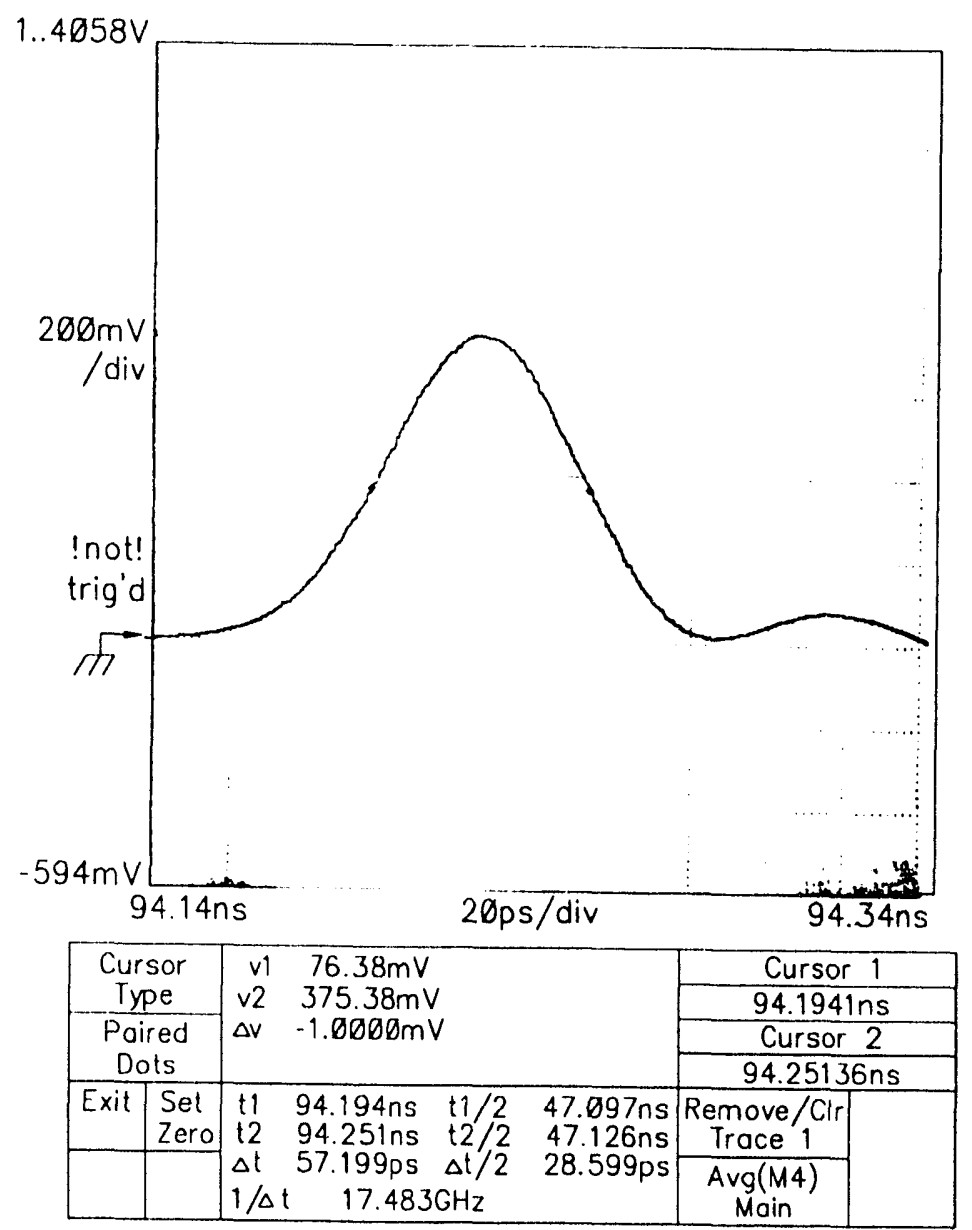

Fig. 10. Waveform of PPL30K generator. 


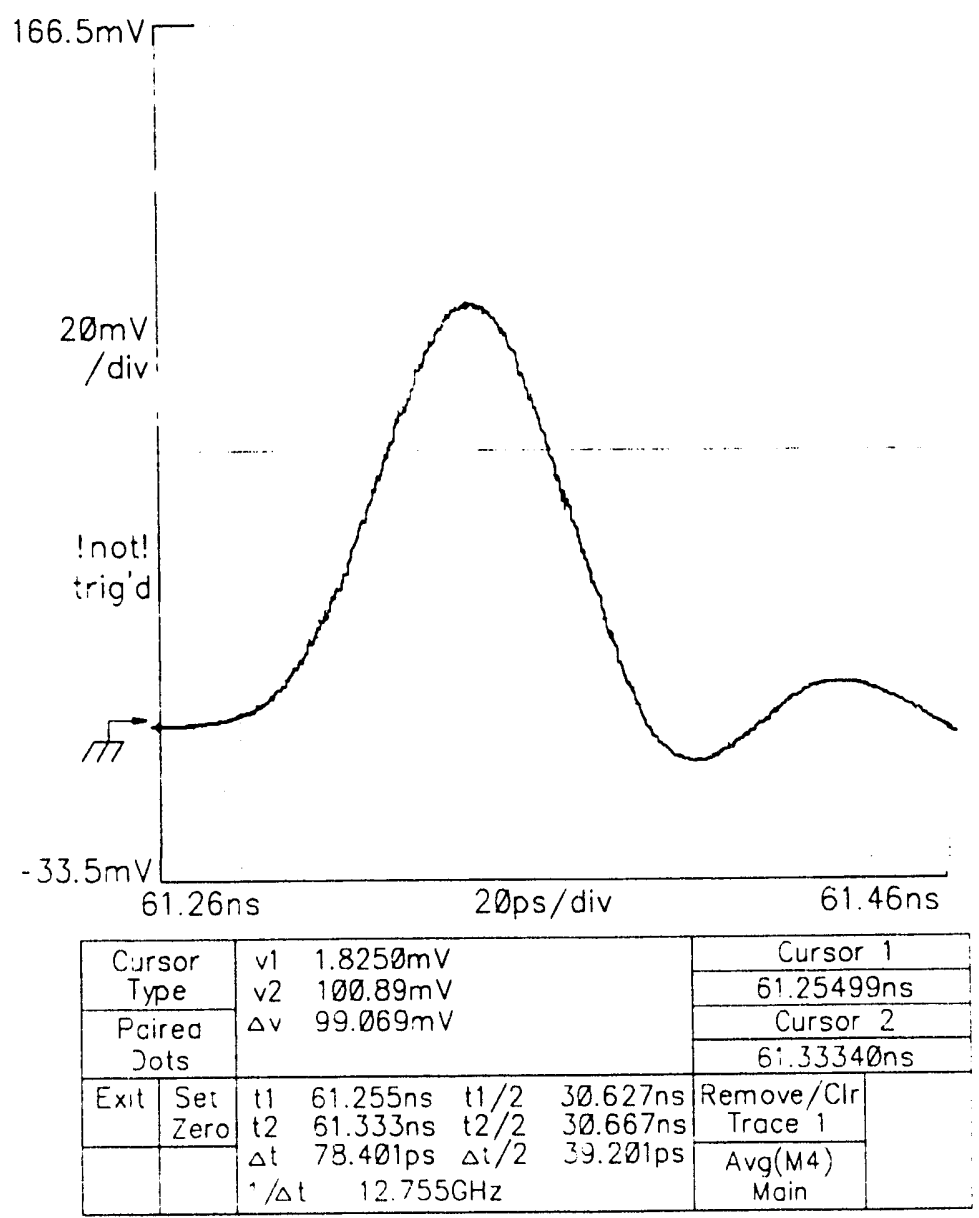

Fig. 11. Waveform of OIG-502 generator. 


\section{EXPERIMENTAL RESULTS}

The first experimental setup is the reflective scheme shown in Figs. 1 and 4. The generator is the Opto-Electronics PPL30K. With no applied strain, the reflections from both ends of the gauge fiber are visible in Fig. 12, the scope trace of September 30, 1991, at 15:11:29. A blowup of the reflection off the near face of the gauge fiber is shown in Fig. 13, the scope trace of September 30,1991, at 15:32:05. A blowup of the reflection off the far face of the gauge fiber is shown in Fig. 14, the scope trace of September 30, 1991, at 15:41:14. The key to the experiment is to be able to measure the time difference between the peaks with picosecond precision. This is illustrated in Fig. 15, the scope trace of October 2, 1991, at 14:25:07. A second trace is generated from the first by adding a small constant. A window is opened on a segment of each trace, allowing the spikes at both ends of the trace to be viewed simultaneously. The time base of the windows is expanded to allow for the precise location of the peaks. The total time difference is found by using a split cursor, one in each window; placing the cursors at the peaks; and letting the scope calculate the time difference between them.

The first question to be answered by the experiments concerned the repeatability of the readings of the time between pulses. Repeatability was observed as follows. For a fiber in the strain gauge apparatus, at a fixed micrometer setting, 20 observations of time of flight were made with two diffe-ent lasers. The results are listed in Table 2. Using the PPL30K laser, the trace was the average of 32 scans. The resulting standard deviation in the 20 consecutive observations is $1.02 \mathrm{ps}$. Using the OIG-502 laser, the trace was the average of 64 scans. The resulting standard deviation in 20 consecutive observations is 1.65 ps.

The implication of these data is that the observation of time between peaks is repeatable to within about \pm 2 ps, using the PPL30K generator. Assuming that the uncertainty in time difference is Gaussian distributed, it is expected that over a large number of readings, the mean value is the correct value, and any individual reading has $95 \%$ probability of being within \pm 2 standard deviations of the mean value. Thus, if the standard deviation of many readings is $1 \mathrm{ps}$, there is approximately $95 \%$ probability that the reading is within \pm 2 ps of the true value.

The slightly poorer performance of the system with the OIG-502 is not surprising. It has one-third the power of the PPL30K. Also, the pulse shape of the OIG-502 is much less symmetrical than the pulse shape of the PPL30K (Figs. 10 and 11). The asymmetry makes it difficult to locate the peak with subpicosecond accuracy.

The first set of time-of-flight vs strain observations was made with the system using the PPL30K generator. The scope traces were made up of 512 samples, and the average of 64 traces was displayed. The fiber span was unsupported and sagged visibly at low tension. As the micrometer is adjusted to lower values, the micrometer screw pushes the stage in the direction that increases tension on the fiber. Thus, the imposed strain moves in the direction opposite the micrometer reading. For the experiment, a micrometer setting of $25.00 \mathrm{~mm}$ was found to be the point at which the fiber became taut and was taken as the zero strain point. The micrometer setting was decreased in increments of $400 \mu \mathrm{m}$ through an elongation range of 0 to $5000 \mu \mathrm{m}$, and the time of flight between peaks was observed at each micrometer setting.

Elongation is the difference between the zero strain micrometer setting of $25.00 \mathrm{~mm}$ and the actual micrometer setting. Strain in pure numbers is the elongation in meters divided by the measured gauge length of $1.3232 \mathrm{~m}$. Strain in microstrains is obtained by multiplying the pure number value of strain by $10^{6}$. 


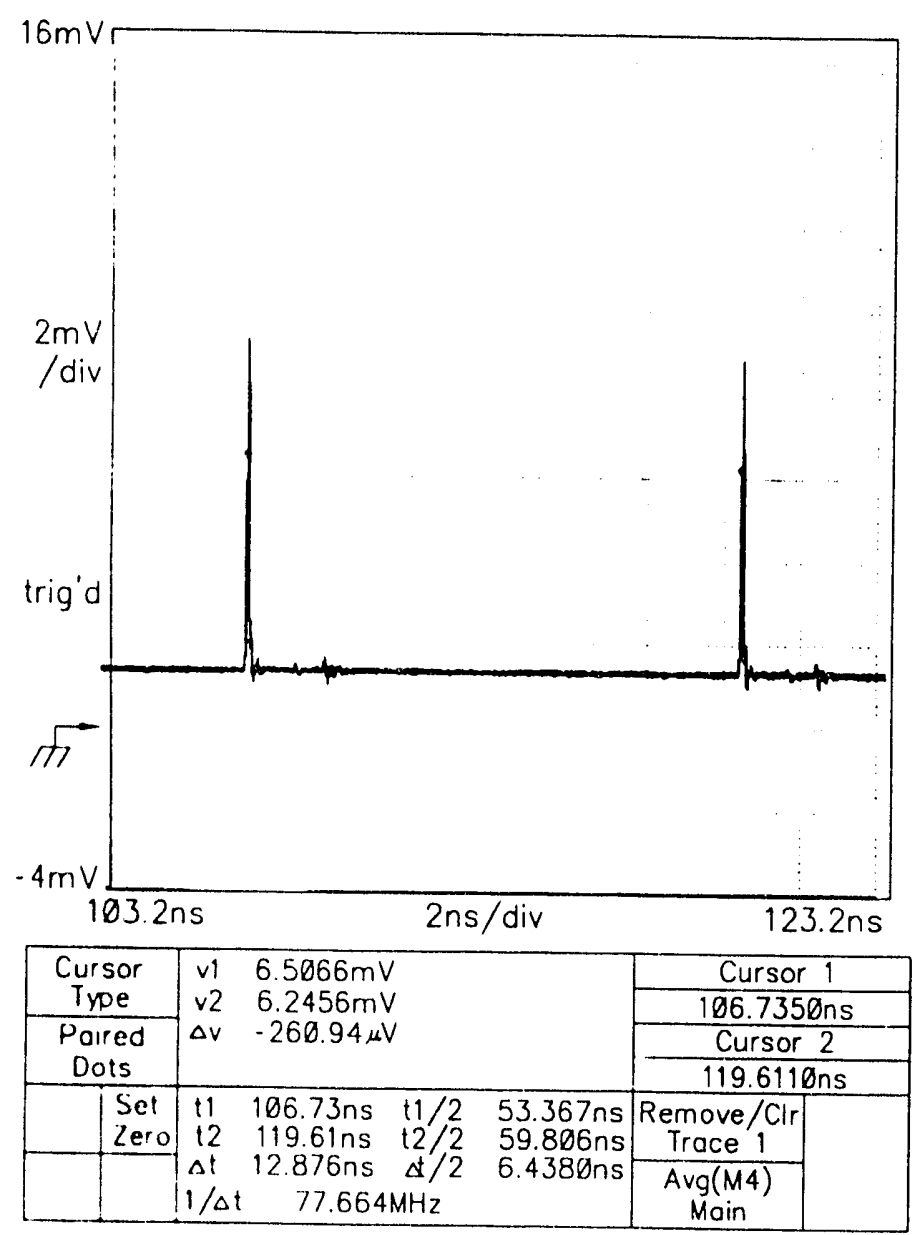

Fig. 12 Main OTDR trace.

Differential time of flight is the difference between the time of flight observed at a given micrometer setting and the time of flight observed at the zero strain setting. Note that the time of flight through the gauge is half the time difference between the two peaks in the scope trace. The reason for this is that, in a reflective setup, the scope trace indicates how long the pulse takes to make two transits of the gauge fiber. Four sets of data are summarized in Table 3. For each set of time of flight vs applied strain data, the corresponding least squares straight line was computed. All four sets of observed data are plotted in Fig. 16. The four least squares lines are plotted in Fig. 17. The observed data points and the least squares lines for each of the four sets of observations are plotted in Figs. 18 to 21 .

Some generalizations about these data can be drawn by considering the error statistics. For this purpose, the least squares line is assumed to be more accurate than the individual observations as a representation of the actual time-of-flight vs strain relationship. Error is defined as the absolute difference between the observed time of flight and the corresponding least squares time of flight. For the data set in Table: 3 , the average error is $0.42 \mathrm{ps}$, the standard deviation in error is $0.30 \mathrm{ps}$, and the maximurn observed error is $1.58 \mathrm{ps}$. Assuming Gaussian statistics, there is $99 \%$ probability of being within three standard deviations of the mean, and for these data the error has $99 \%$ probability of being within 


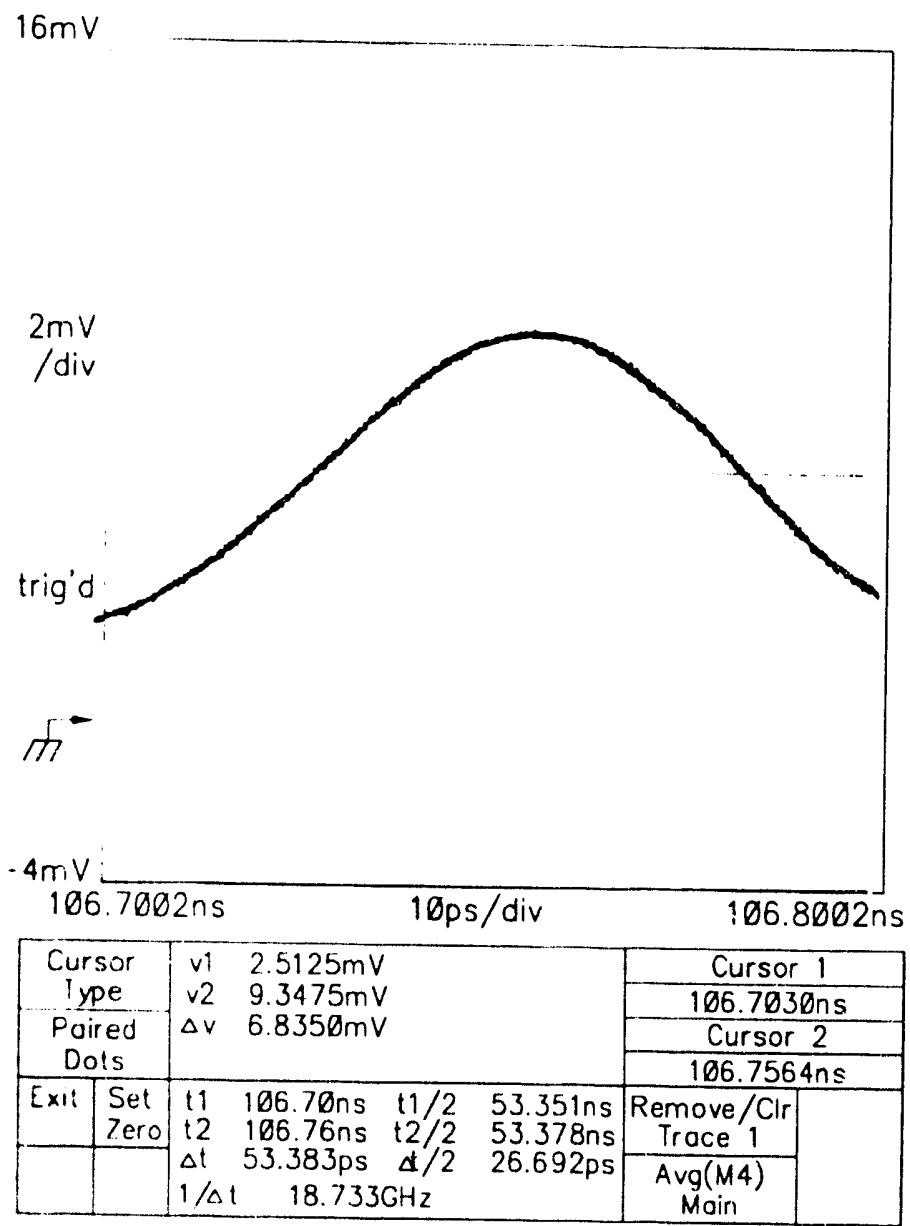

Fig. 13. Near-face reflection.

$\pm(0.42+3 \times 0.30)= \pm 1.32 \mathrm{ps}$. This is well within the \pm 2 ps of uncertainty suggested by the data in Table 2 .

The second set of time-of-flight vs strain observations was made with the system using the PPL30K generator. The scope traces were made up of 512 samples, and the average of 32 traces was displayed. The fiber span was continuously supported to eliminate catenary effects. As the micrometer is adjusted to lower values, the micrometer screw pushes the stage in the direction that increases tension on the fiber. Thus, the imposed strain moves in the direction opposite the micrometer reading. For the experiment, a micrometer setting of $25.00 \mathrm{~mm}$ was found to be the point at which the fiber became taut and was taken as the zero strain point. The micrometer setting was decreased in increments of $400 \mu \mathrm{m}$ through an elongation range of 0 to $5000 \mu \mathrm{m}$, and the time of flight between peaks was observed at each micrometer setting.

Elongation is the difference between the zero strain micrometer setting of $25.00 \mathrm{~mm}$ and the actual micromete: setting. Strain in pure numbers is the elongation in meters divided by the measured gauge length of $1.3232 \mathrm{~m}$. Strain in microstrains is obtained by multiplying the pure number value of strain by $10^{6}$. 


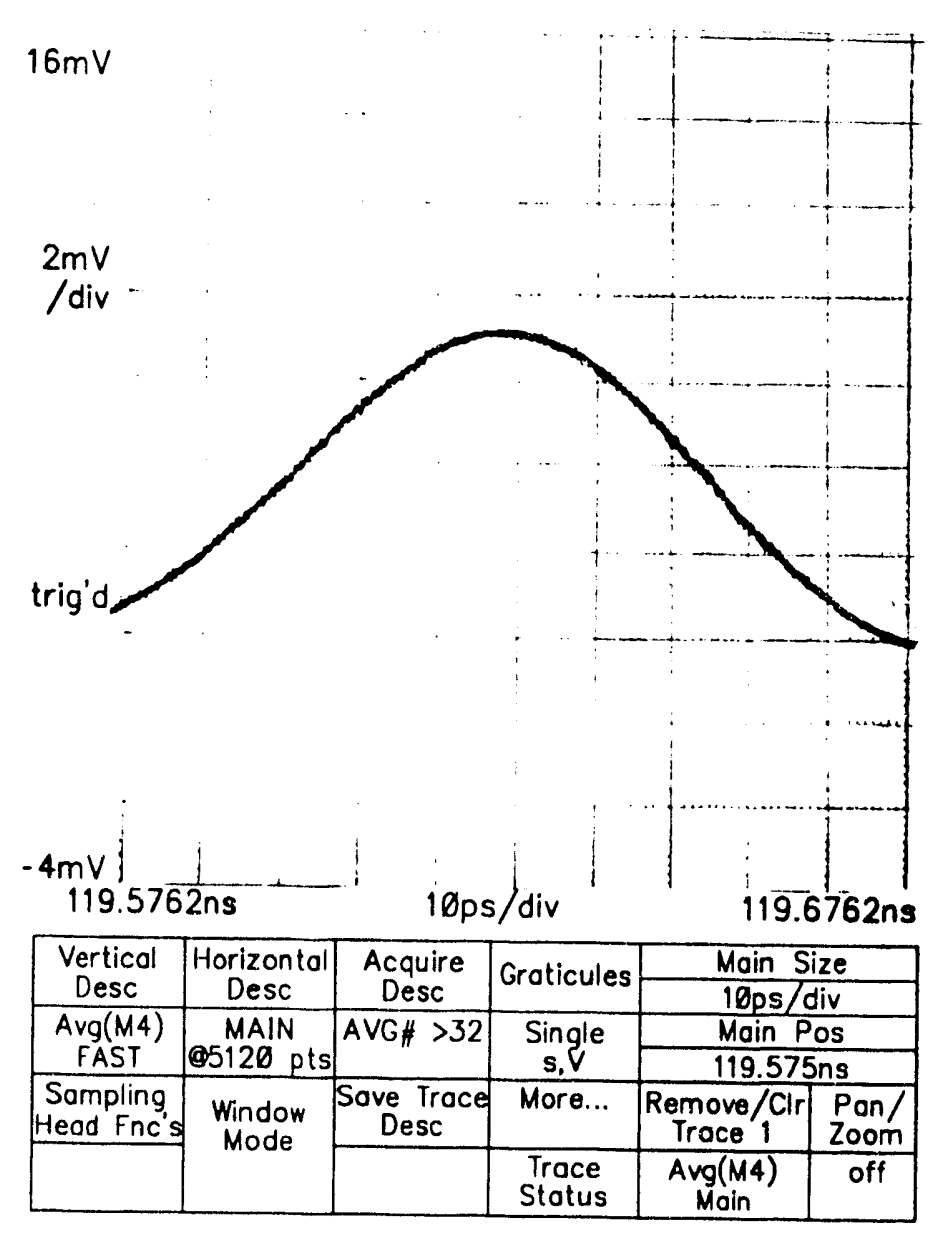

Fig. 14. Far-face reflection.

Differential time of flight is the difference in the time of flight observed at a given micrometer setting and the time of flight observed at the zero strain setting. Note that the time of flight through the gauge is half the time difference in the two peaks in the scope trace. The reason for this is that, in a reflective setup, the scope trace indicates how long the pulse takes to make two transits of the gauge fiber. Four sets of data are summarized in Table 4. For each set of time-of-flight vs applied strain data, the corresponding least squares straight line was computed. All four sets of observed data are plotted in Fig. 22. The four least squares lines are plotted in Fig. 23. The observed data points and the least squares lines for each of the four sets of observations are plotted in Figs. 24 to 27.

Some generalizations about these data can be drawn by considering the error statistics. For this purpose, the least squares line is assumed to be more accurate than the individual observations as a representation of the actual time-of-flight vs strain relationship. Error is defined as the absolute difference between the observed time of flight and the corresponding least squares time of flight. For the data set in Table 4 the average error is $0.44 \mathrm{ps,} \mathrm{the}$ standard deviation in error is $0.31 \mathrm{ps,}$, and the maximum observed error is $1.49 \mathrm{ps}$. If there is 99\% probability of being within three standard deviations of the mean, then, for these data, 

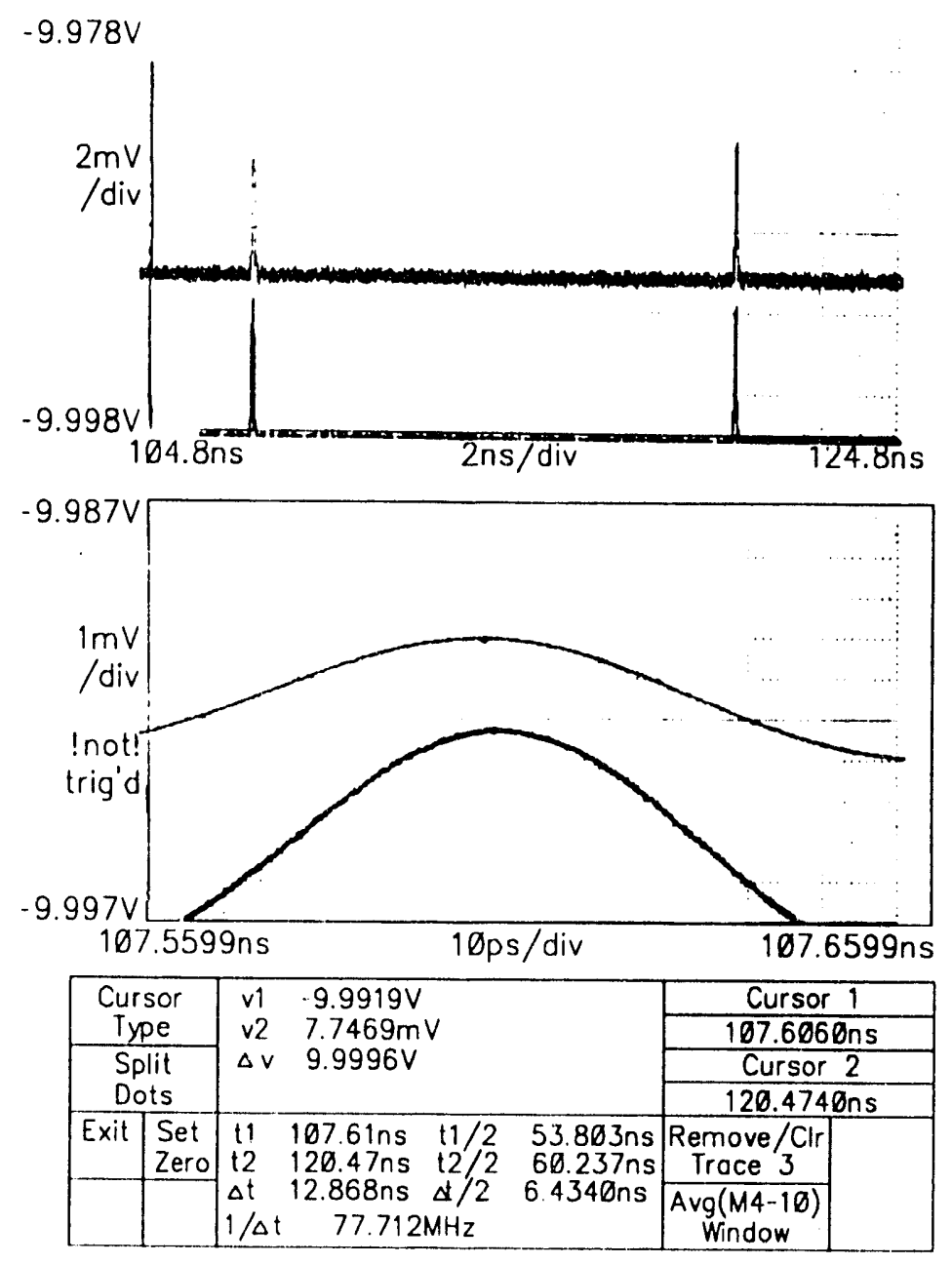

Fig. 15. Measurement screen.

the error in the data has $99 \%$ probability of being within $\pm(0.44+3 \times 0.31)= \pm 1.37$ ps. This is well within the \pm 2 ps of uncertainty suggested by the data in Table 2 .

The third set of time-of-flight vs strain observations was made with the system using the OIG-502 generator in high power mode with a $10-\mathrm{kHz}$ repetition rate. The scope traces were made up of 512 samples, and the average of 64 traces was displayed. The fiber span was continuously supported to eliminate catenary effects. As the micrometer is adjusted to lower values, the micrometer screw pushes the stage in the direction that increases tension on the fiber. Thus, the imposed strain moves in the direction opposite the micrometer reading. For the experiment, a micrometer setting of $25.00 \mathrm{~mm}$ was found to be the point at which the fiber became taut and was taken as the zero strain point. The micrometer setting was decreased in increments of $400 \mu \mathrm{m}$ through an elongation range of 0 to $5000 \mu \mathrm{m}$, and the time of flight between peaks was observed at each micrometer setting.

Elongation is the difference between the zero strain micrometer setting of $25.00 \mathrm{~mm}$ and the actual micrometer setting. Strain in pure numbers is the elongation in meters divided by the measured gauge length of $1.3232 \mathrm{~m}$. Strain in microstrains is obtained by multiplying the pure number value of strain by $10^{6}$. 
Table 2. Repeatability of OTDR readings-comparison of Tektronix OIG-502 and Opto-Electronics PPL30K generators

\begin{tabular}{ccc}
\hline & \multicolumn{2}{c}{ Time of flight between peaks (ps) } \\
\cline { 2 - 3 } Observation & PPL30K & OIG-502 \\
\hline 1 & 12,721 & 12,724 \\
2 & 12,720 & 12,727 \\
3 & 12,722 & 12,724 \\
4 & 12,722 & 12,727 \\
5 & 12,722 & 12,727 \\
6 & 12,723 & 12,725 \\
7 & 12,723 & 12,722 \\
8 & 12,721 & 12,725 \\
9 & 12,721 & 12,723 \\
10 & 12,721 & 12,726 \\
11 & 12,723 & 12,726 \\
12 & 12,723 & 12,722 \\
13 & 12,721 & 12,726 \\
14 & 12,721 & 12,727 \\
15 & 12,723 & 12,726 \\
16 & 12,722 & 12,725 \\
17 & 12,720 & 12,724 \\
18 & 12,722 & 12,724 \\
19 & 12,720 & 12,725 \\
20 & 12,721 & 12,728 \\
& 12,723 & 12,728 \\
Mange & 12,720 & 12,722 \\
Mean & 3 & 6 \\
Standard deviation & $12,721.6$ & $12,725.15$ \\
Max & 1.02 & 1.65 \\
Maximum & $12,723.63$ & $12,728.45$ \\
Manimum & $12,719.56$ & $12,721.84$ \\
\hline
\end{tabular}

Differential time of flight is the difference in the time of flight observed at a given micrometer setting and the time of flight observed at the zero strain setting. Note that the time of flight through the gauge is half the time difference in the two peaks in the scope trace. The reason for this is that, in a reflective setup, the scope trace indicates how long the pulse takes to make two transits of the gauge fiber. Six sets of data are summarized in Table 5. For each set of time-of-flight vs applied strain data, the corresponding least squares straight line was computed. All six sets of observed data are plotted in Fig. 28. The six least squares lines are plotted in Fig. 29. The observed data points and the least squares lines for each of the six sets of observations are plotted in Figs. 30 to 35.

Some generalizations about these data can be drawn by considering the error statistics. For this purpose, the least squares line is assumed to be more accurate than the individual observations as a representation of the actual time-of-flight vs strain relationship. Error is defined as the absolute difference between the observed time of flight and the corresponding least squares time of flight. For the data set in Table 5 the average error is $0.43 \mathrm{ps, \text {the }}$ 


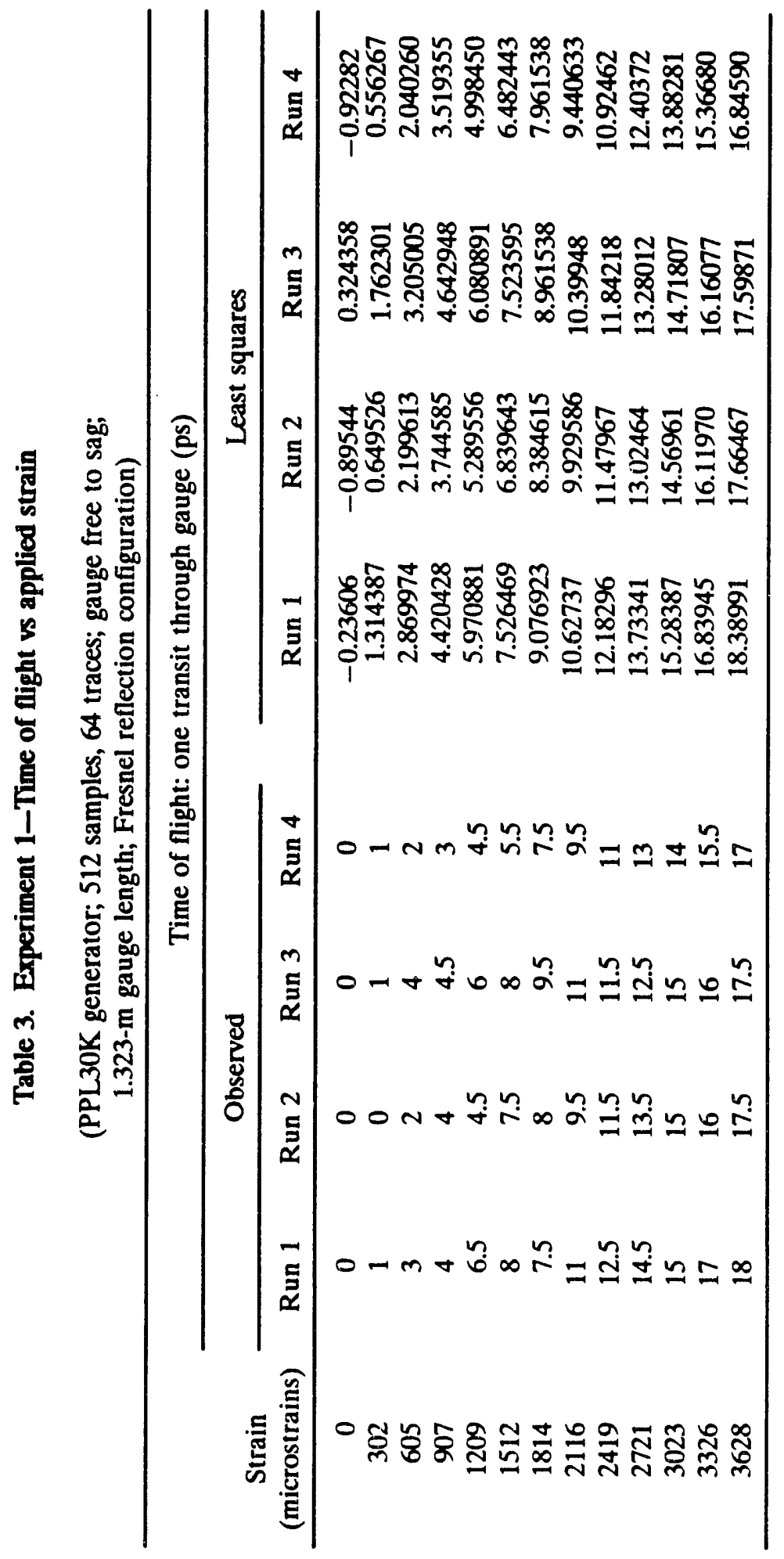




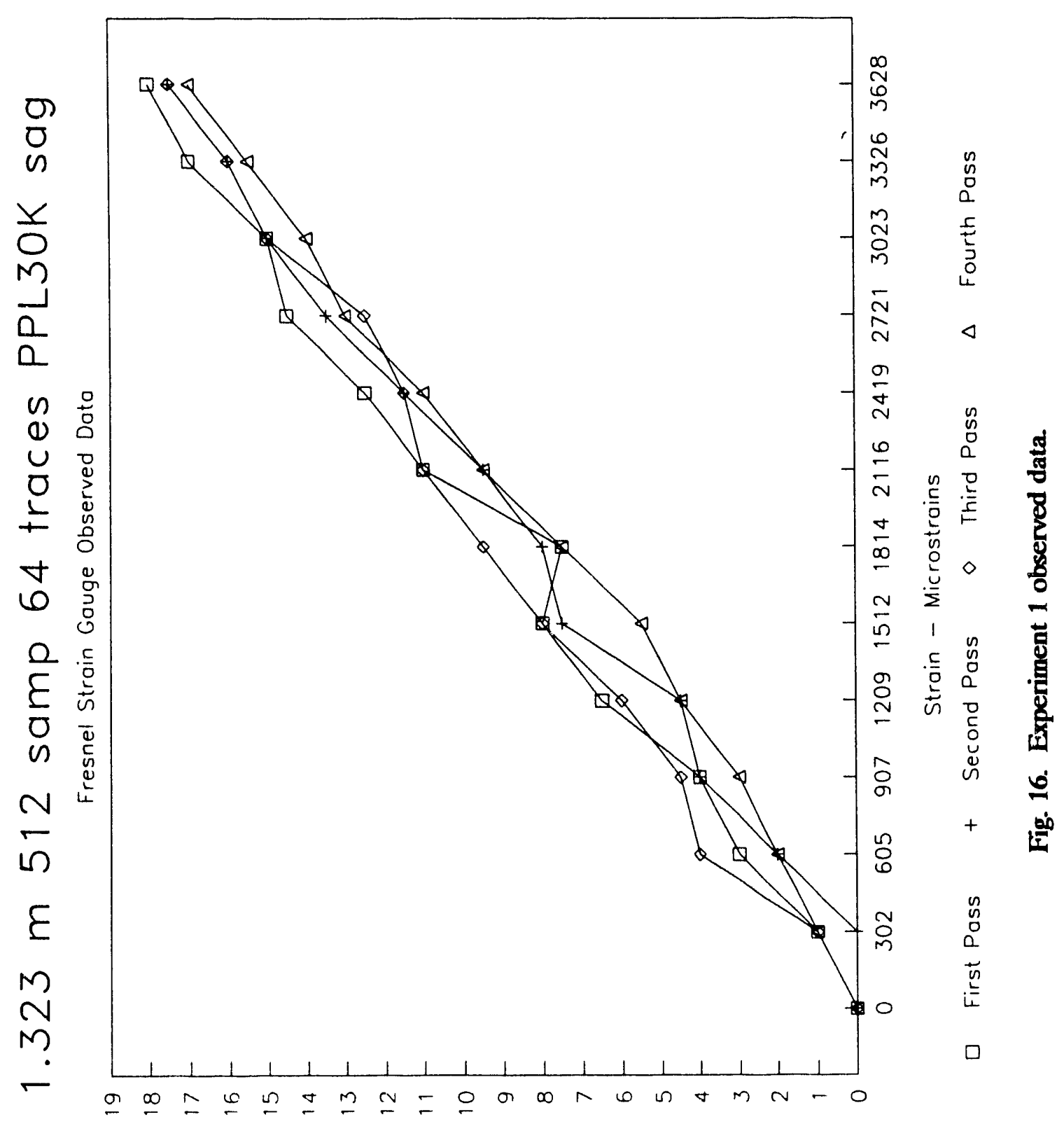

sd - 746!1 to am!1 10!7uasa11!0 


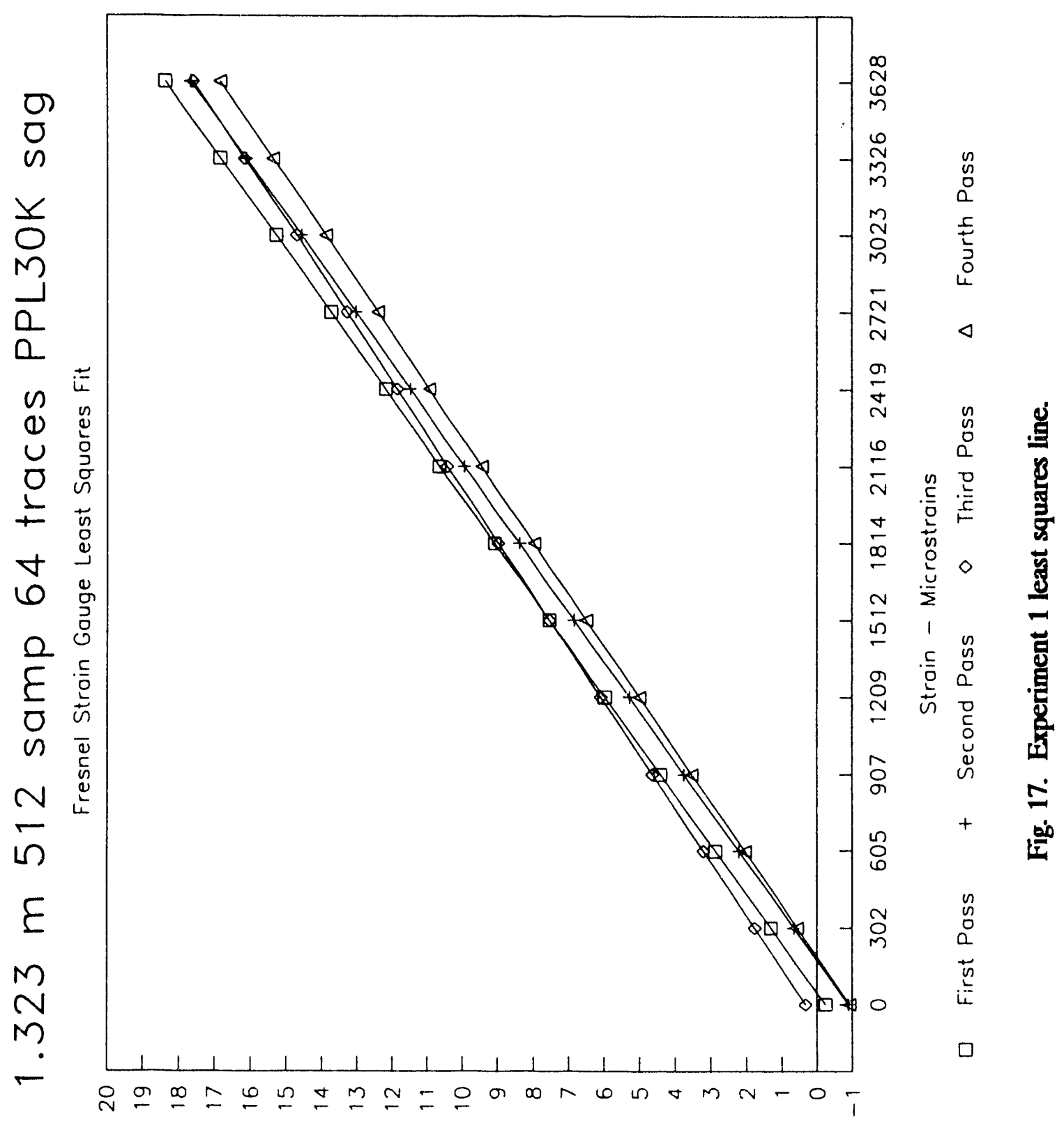

so - 746!1] 10 au!1 10!7uadapt!0 


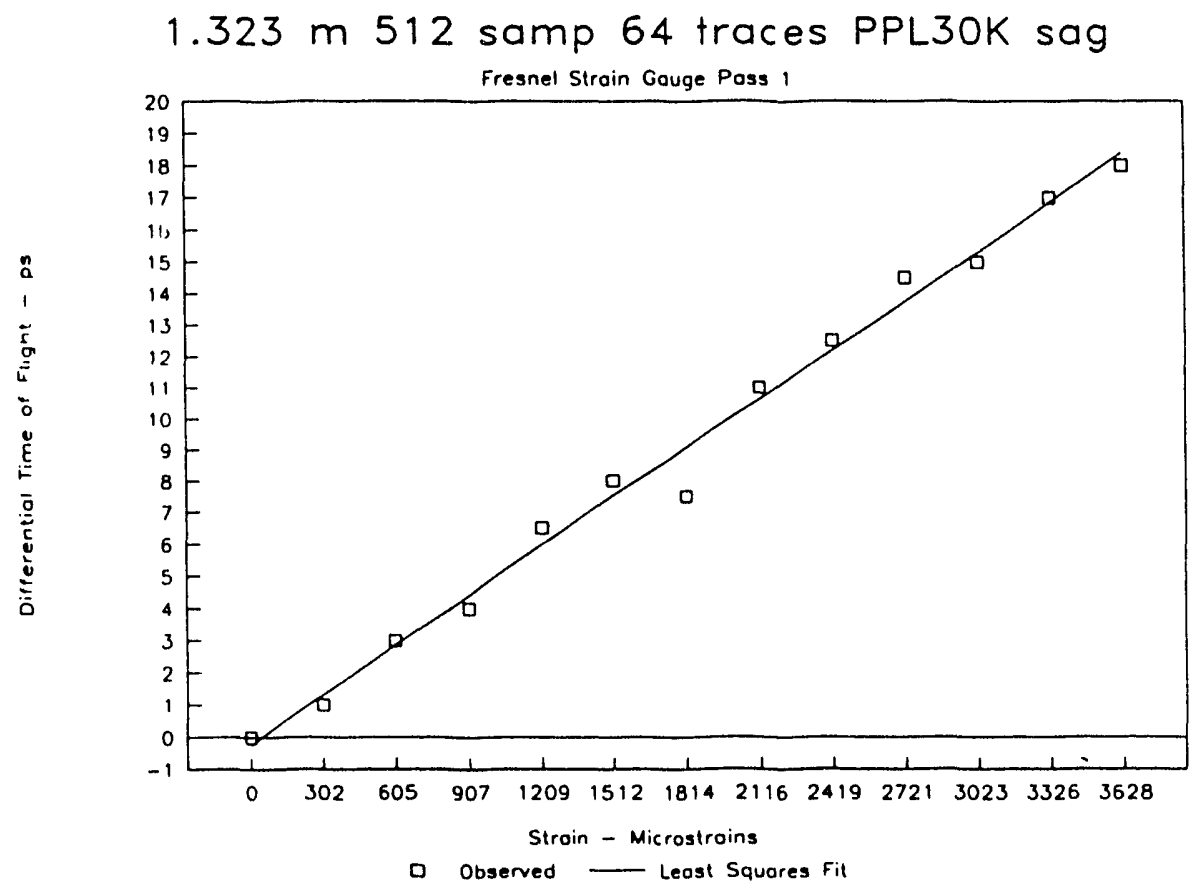

Fig. 18. Experiment 1, run 1.

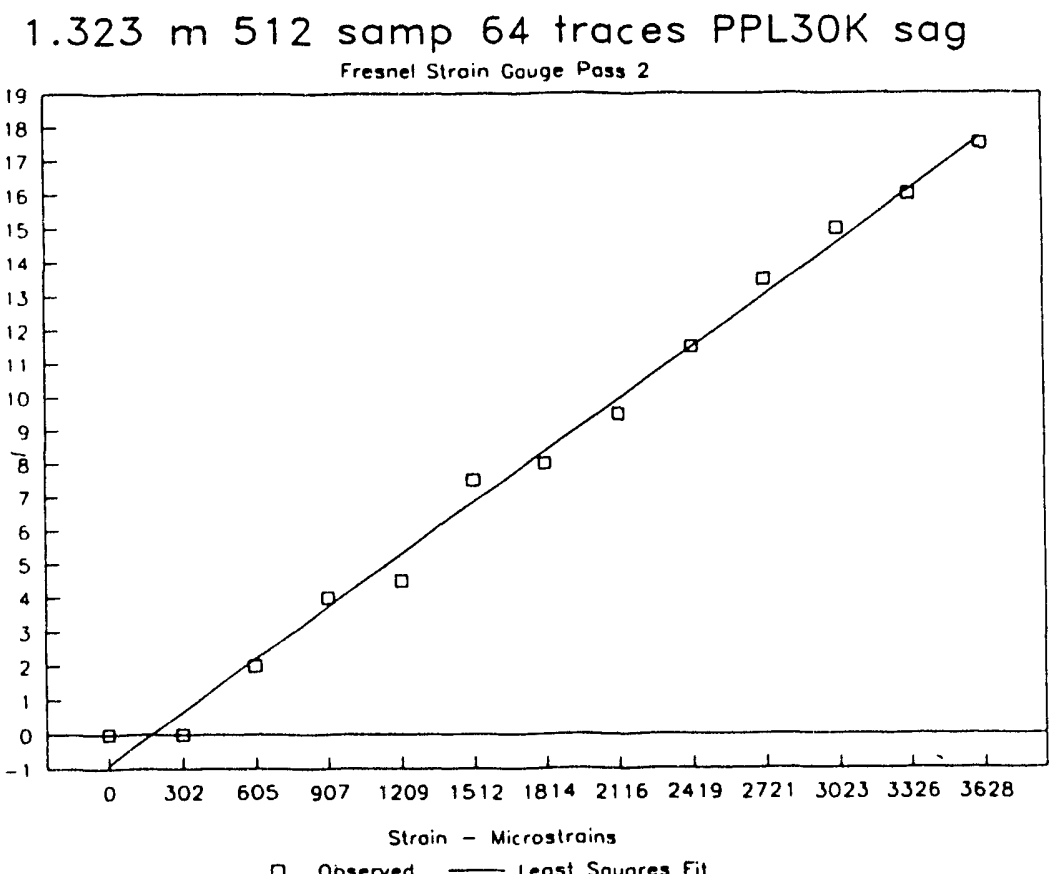

Fig. 19. Experiment 1, run 2 


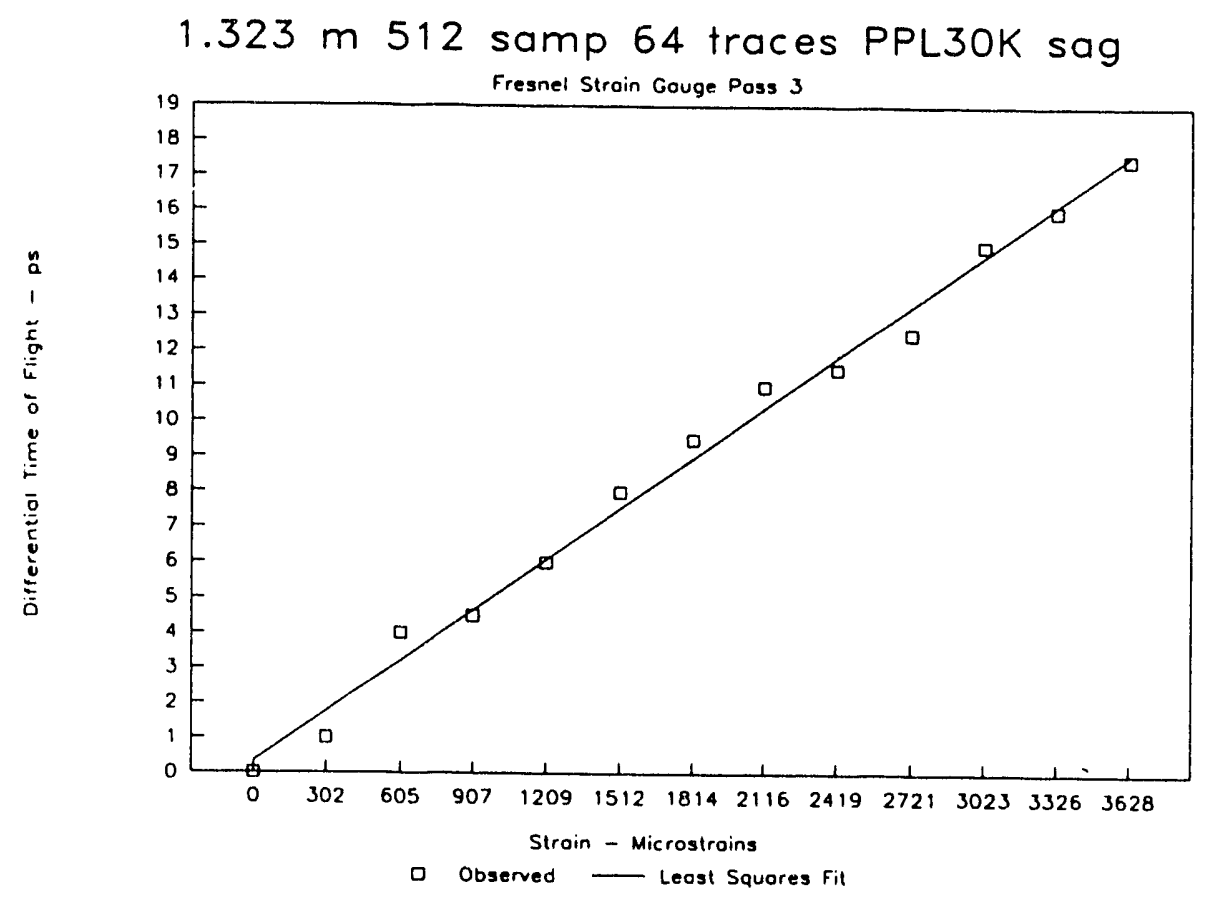

Fig. 20. Experiment 1, run 3.

\section{$1.323 \mathrm{~m} 512$ samp 64 traces PPL3OK sag}

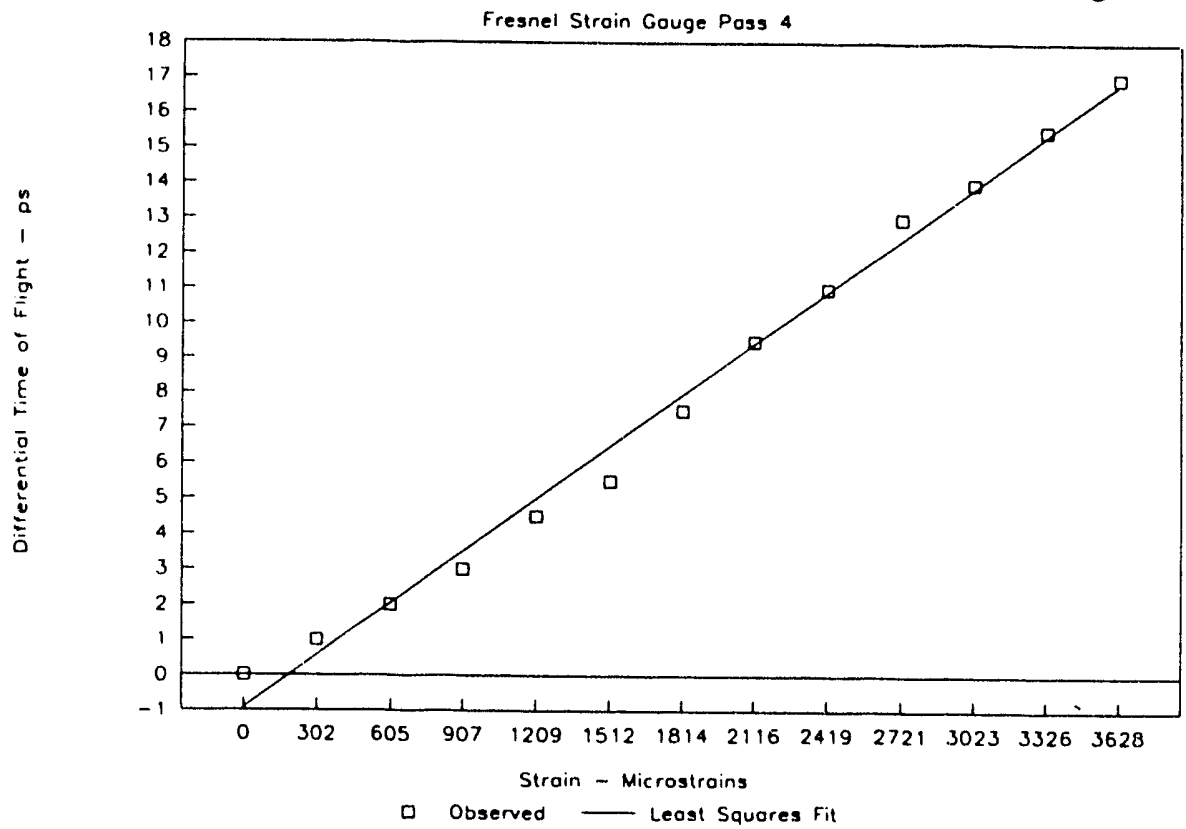

Fig. 21. Experiment 1, run 4. 


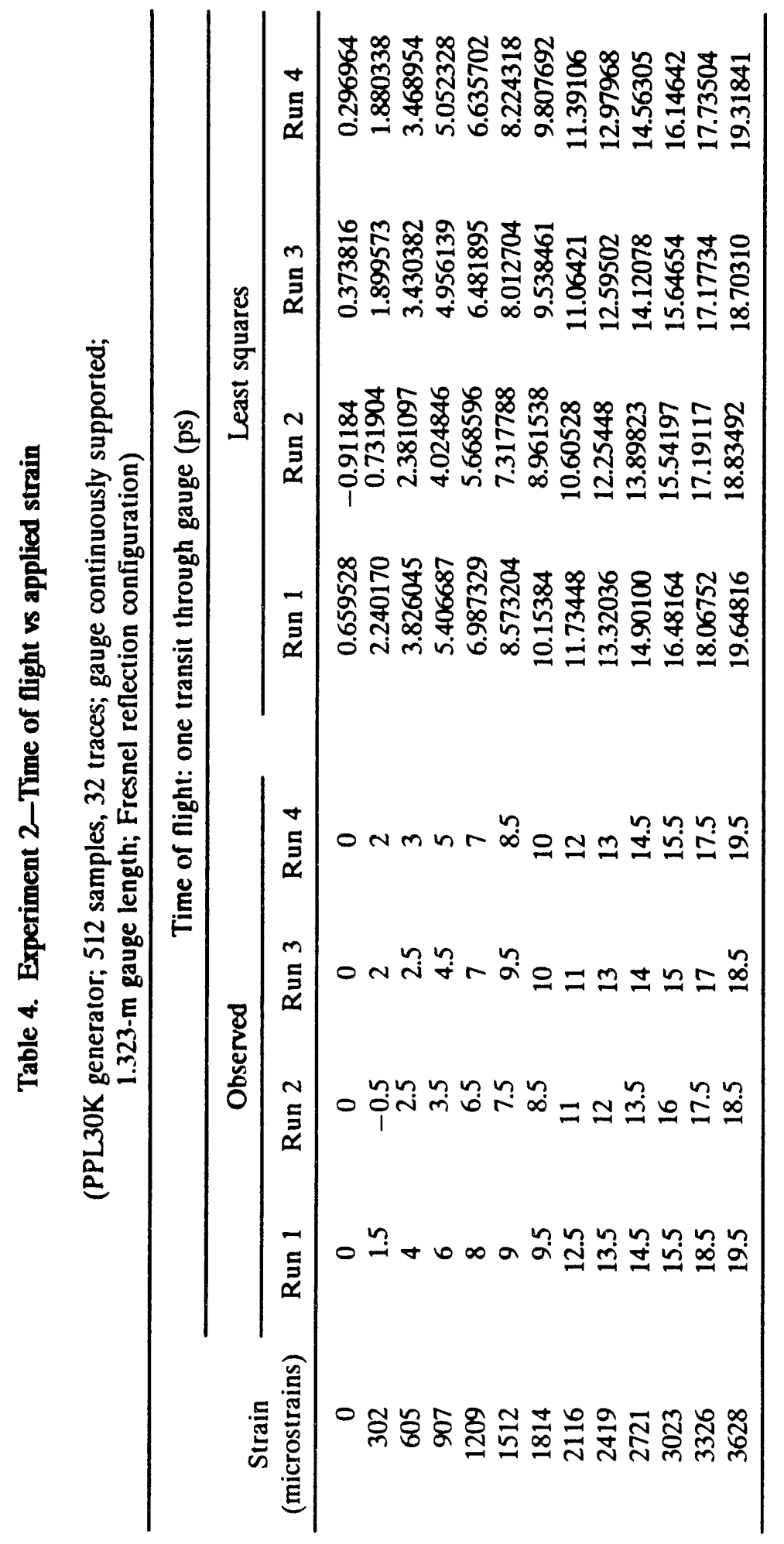




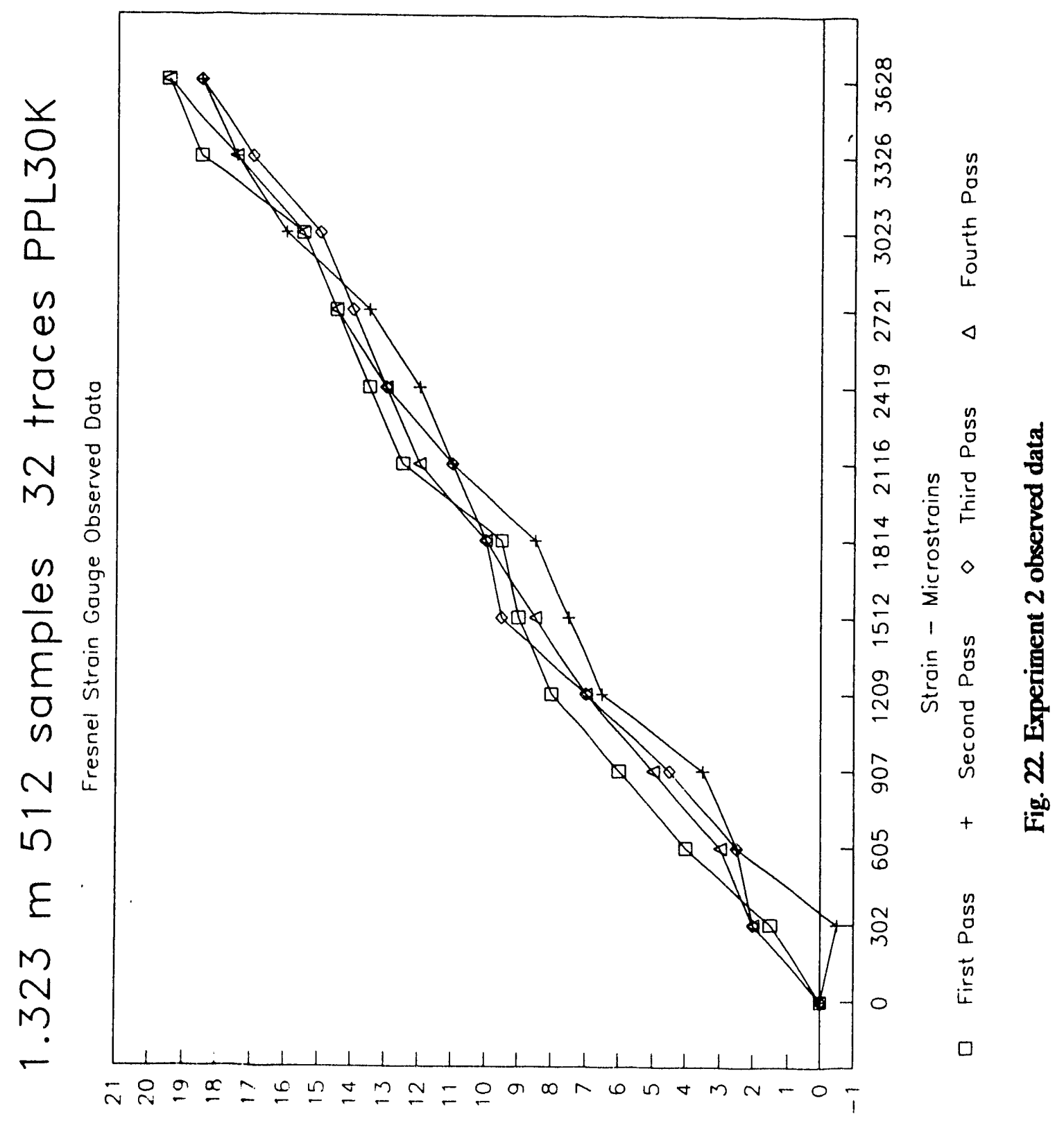

20 - 74D!17 to am!n 10!7uajath!0 


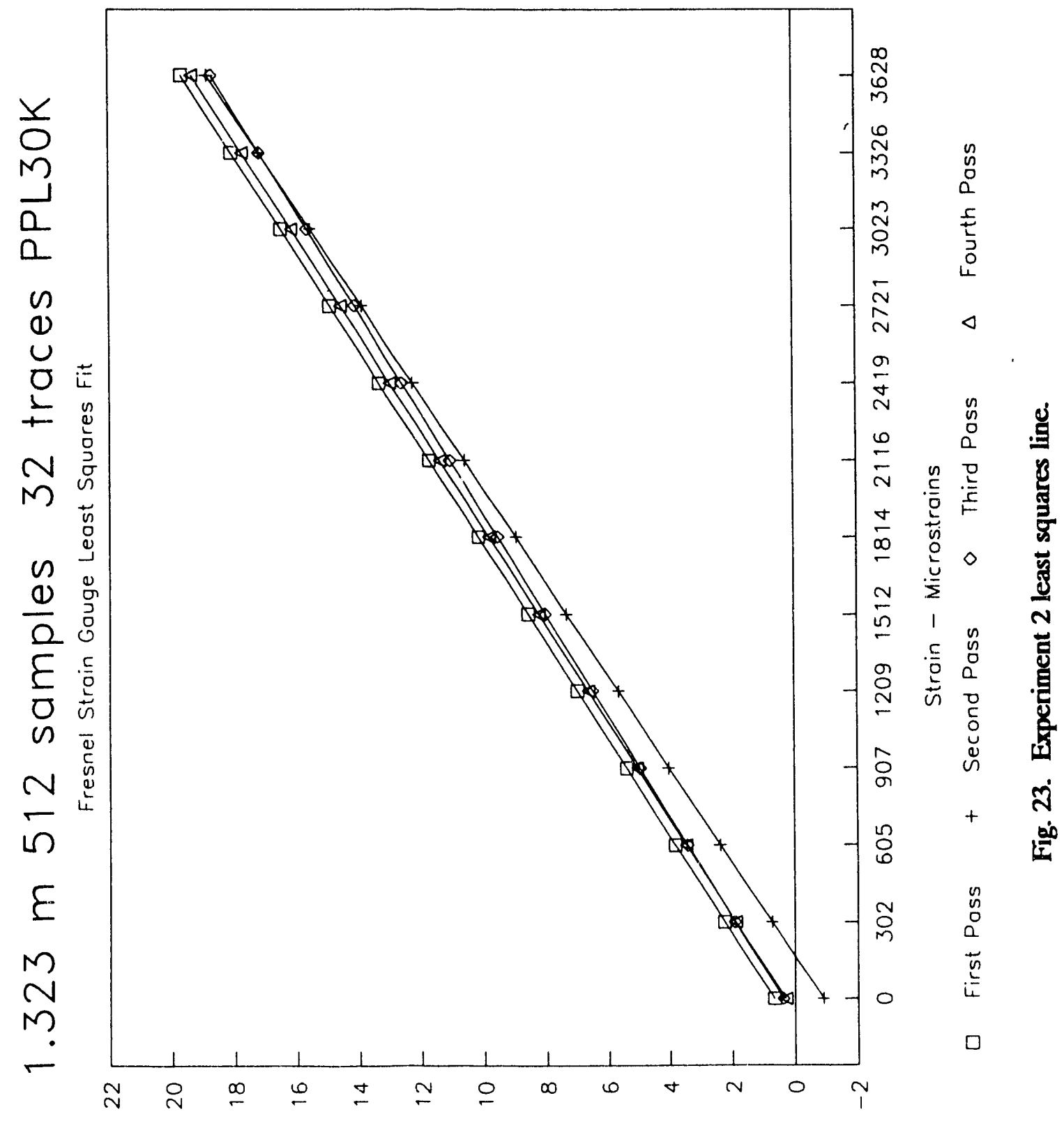

so - 746!!f to am!n 10!7uasaht!0 
$1.323 \mathrm{~m} 512$ samples 32 traces PPL3OK

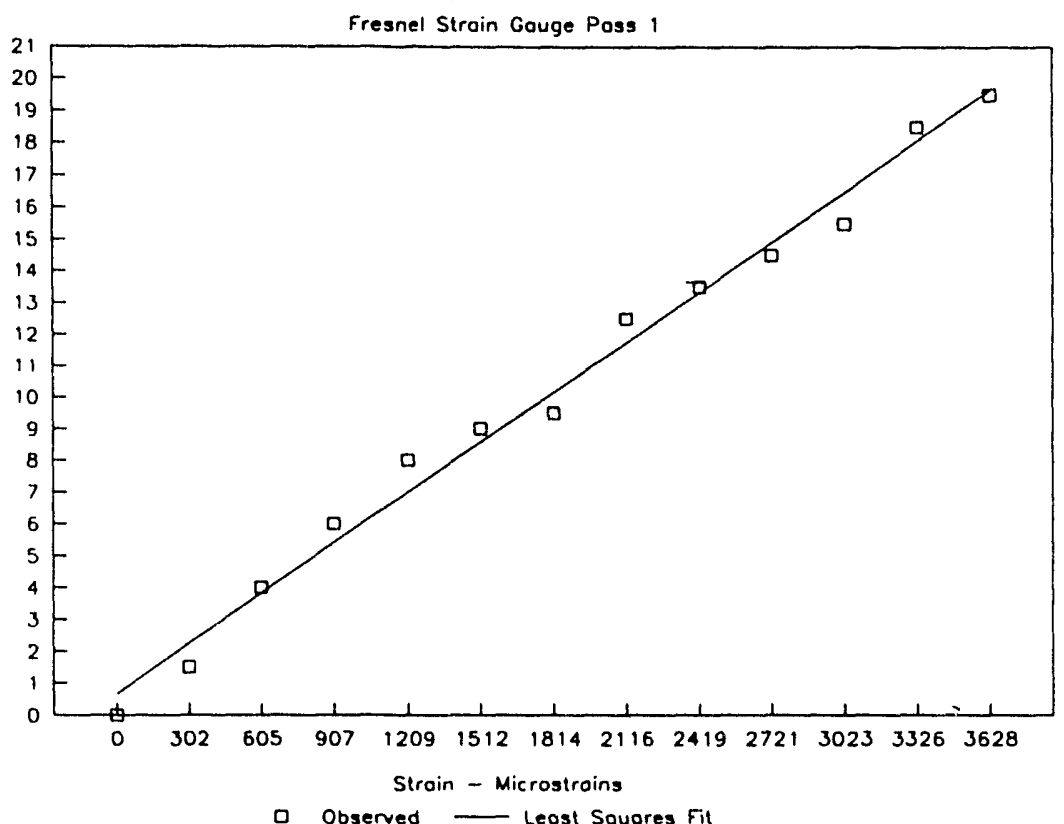

Fig. 24. Experiment 2, run 1.

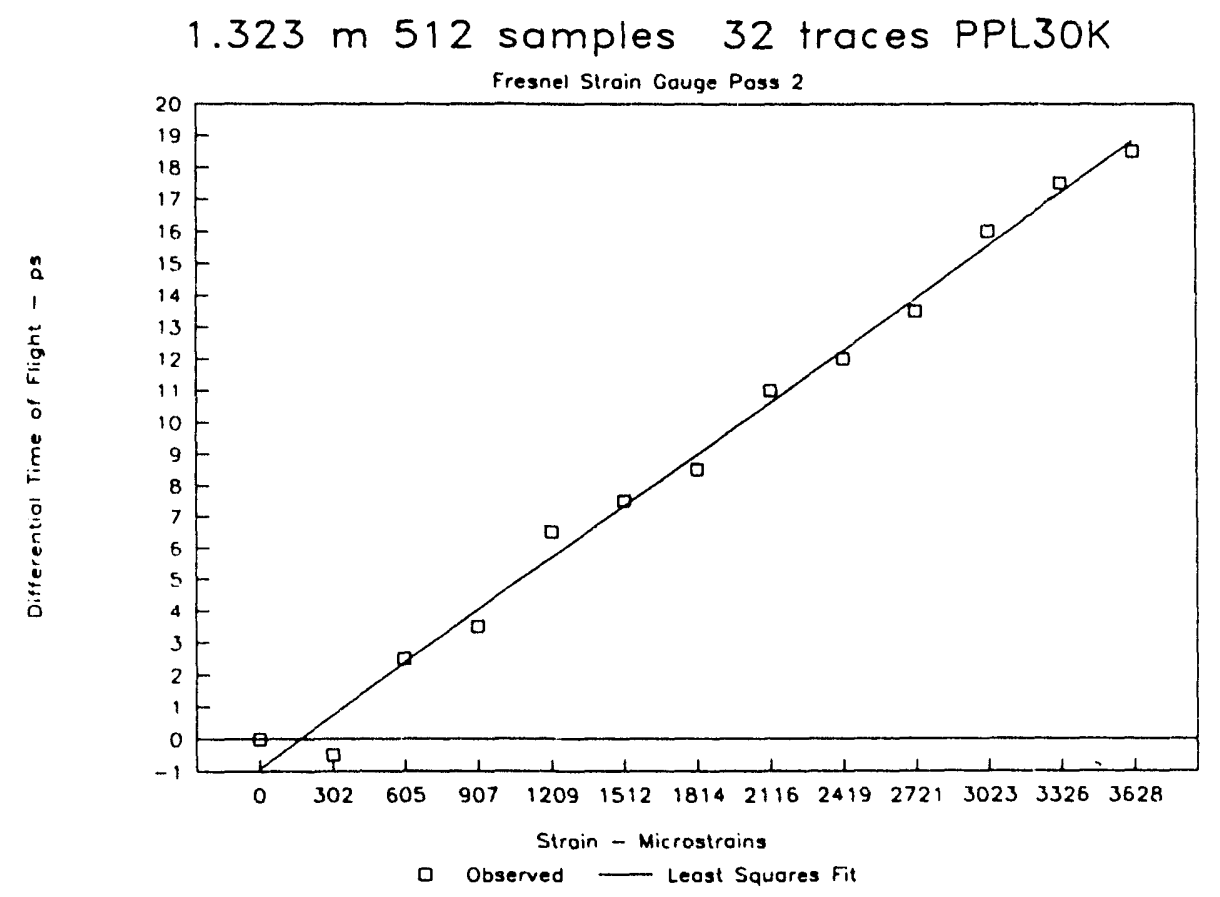

Fig. 25. Experiment 2, run 2. 


\section{$1.323 \mathrm{~m} 512$ samples 32 traces PPL3OK}

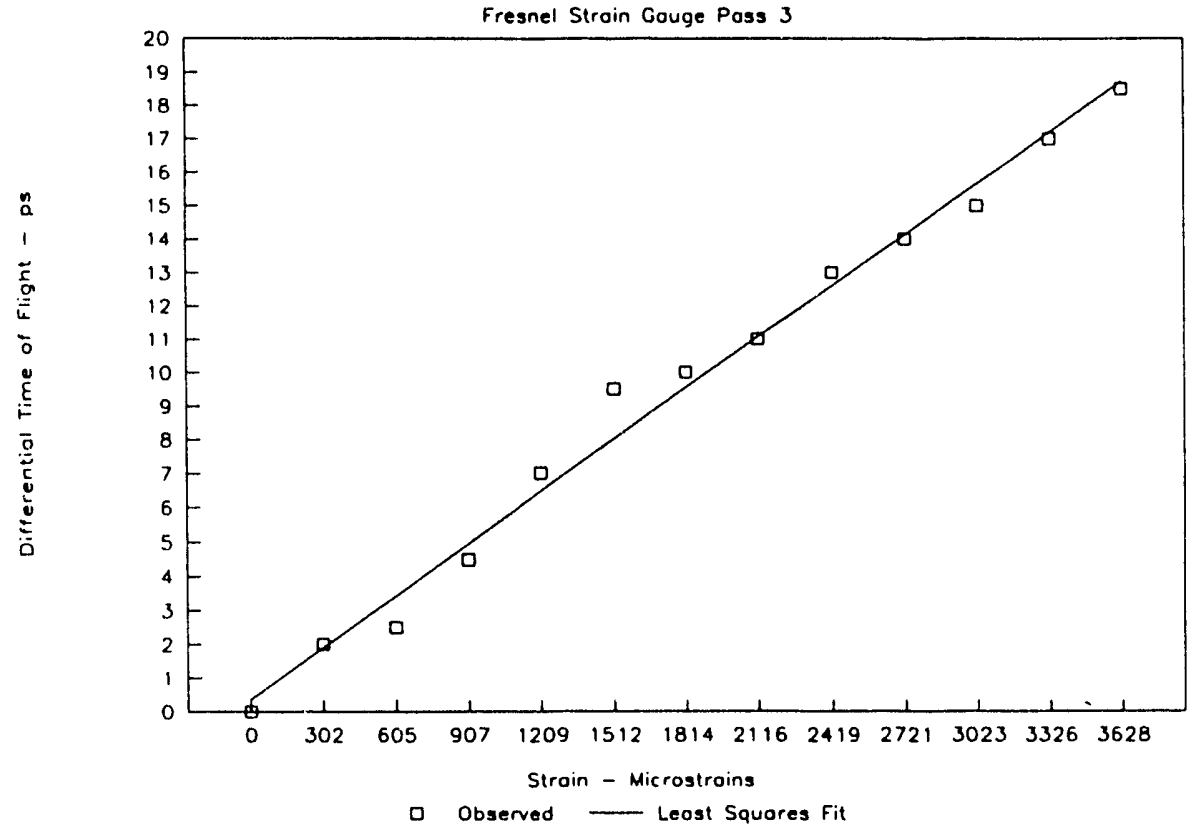

Fig. 26. Experiment 2, run 3.

\section{$1.323 \mathrm{~m} 512$ samples 32 traces PPL3OK} Fresnel Strain Gauge Pass 4

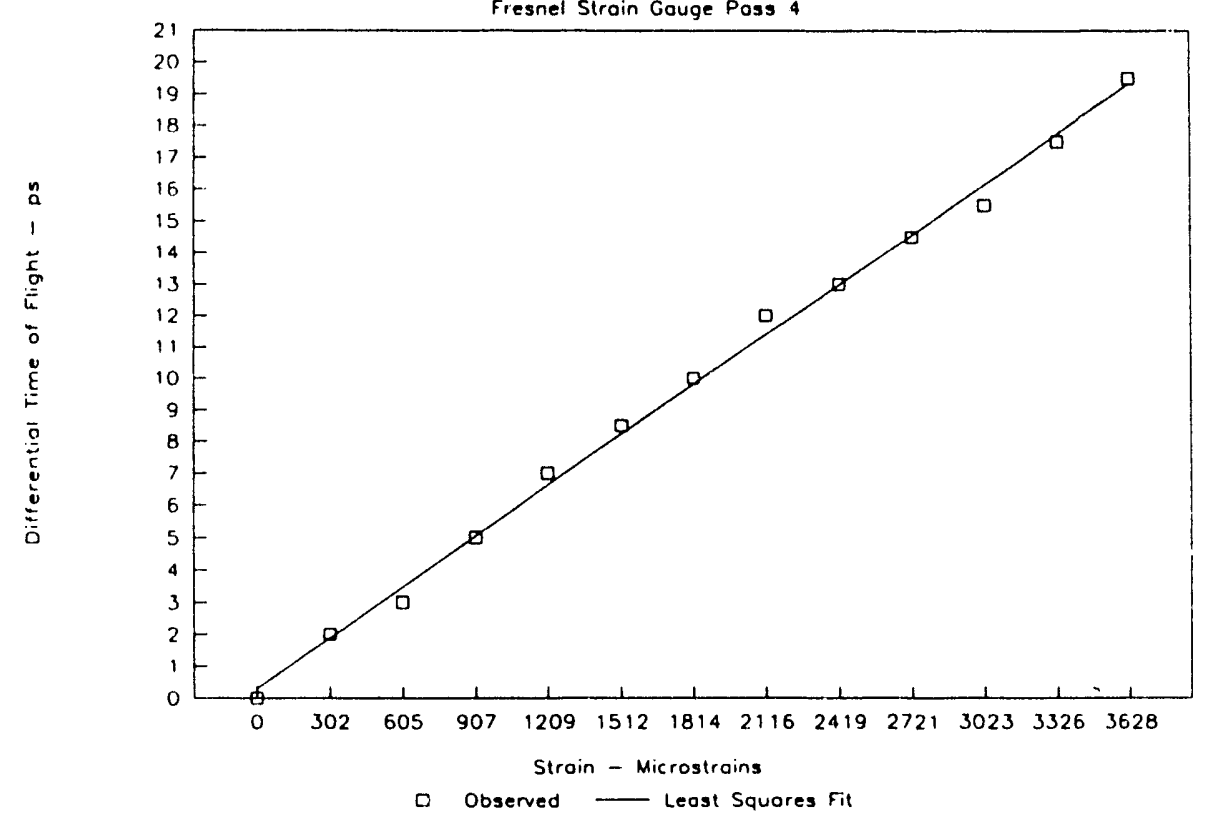

Fig. 27. Experiment 2, run 4. 


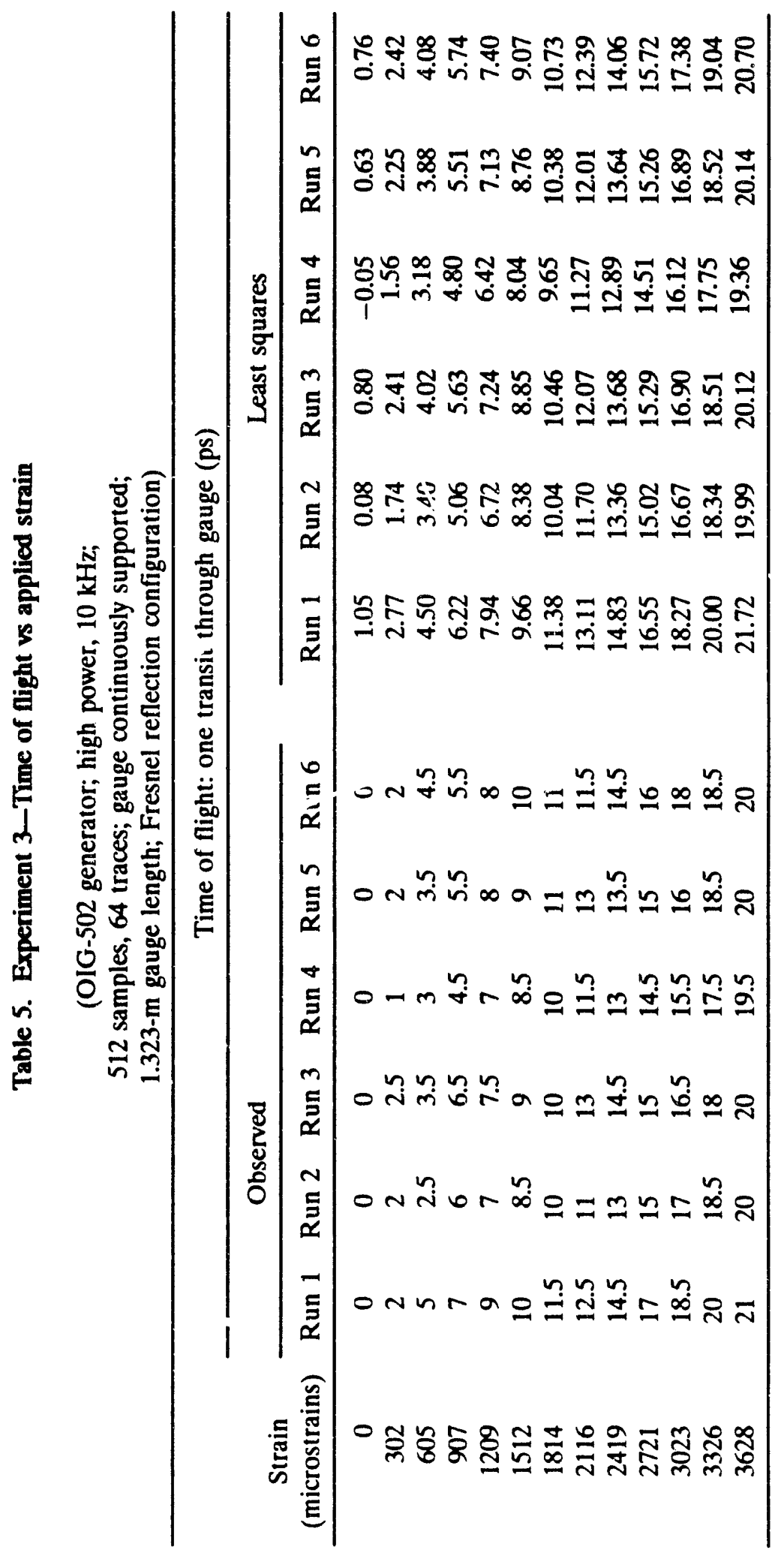




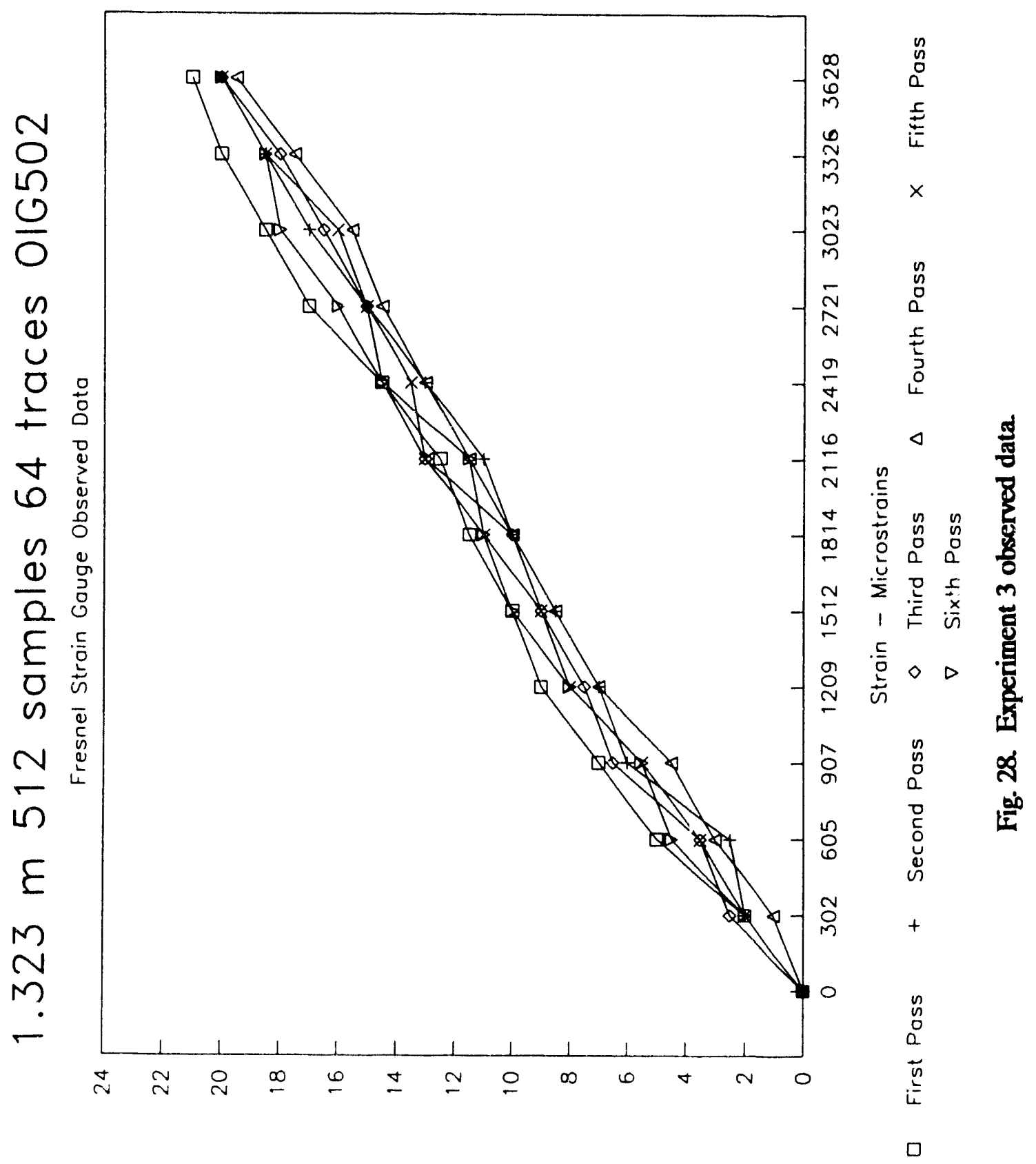

sd - 745!1] to am!1 10!7uadatt!0 


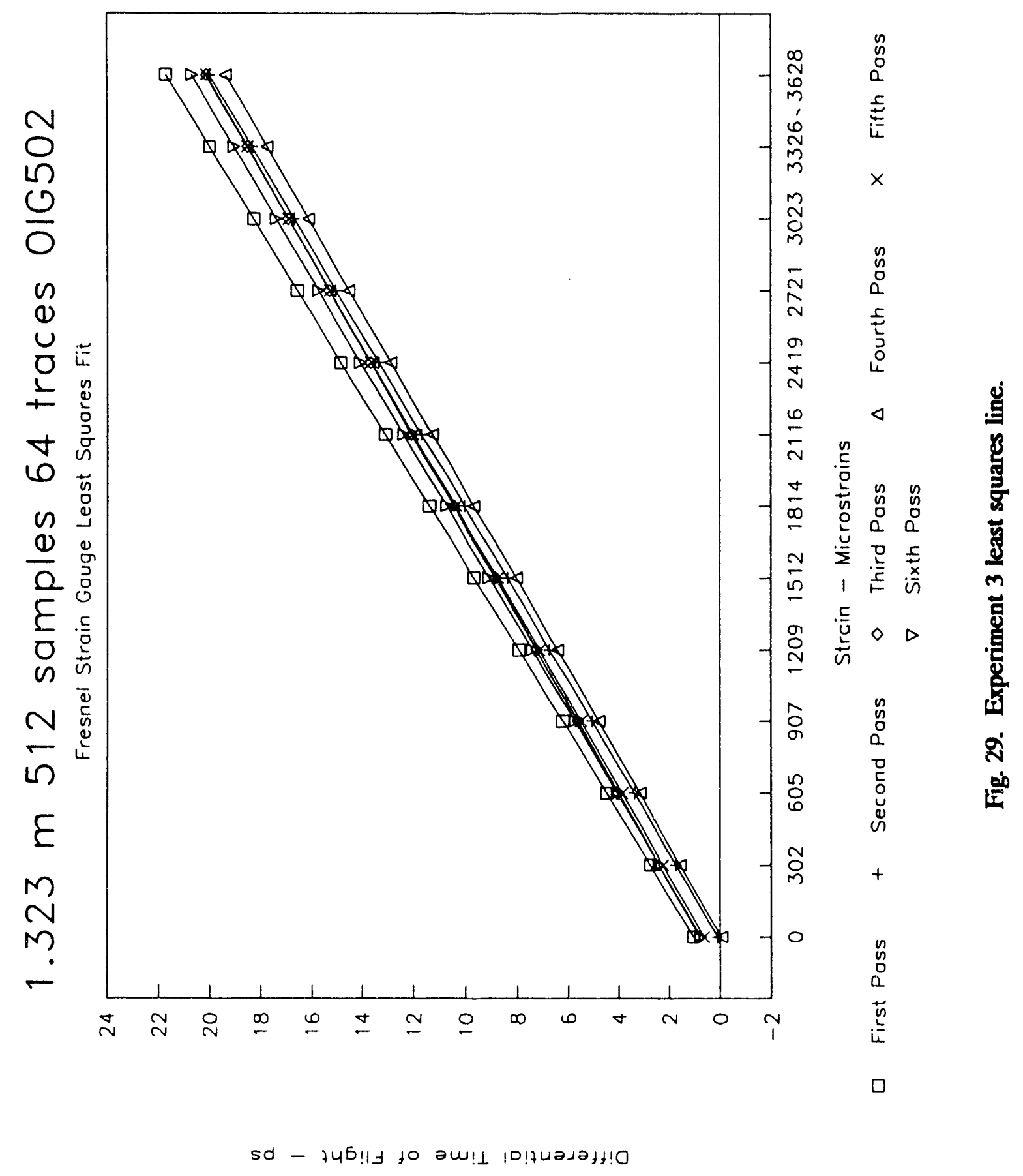


$1.323 \mathrm{~m} 512$ samples 64 traces 016502

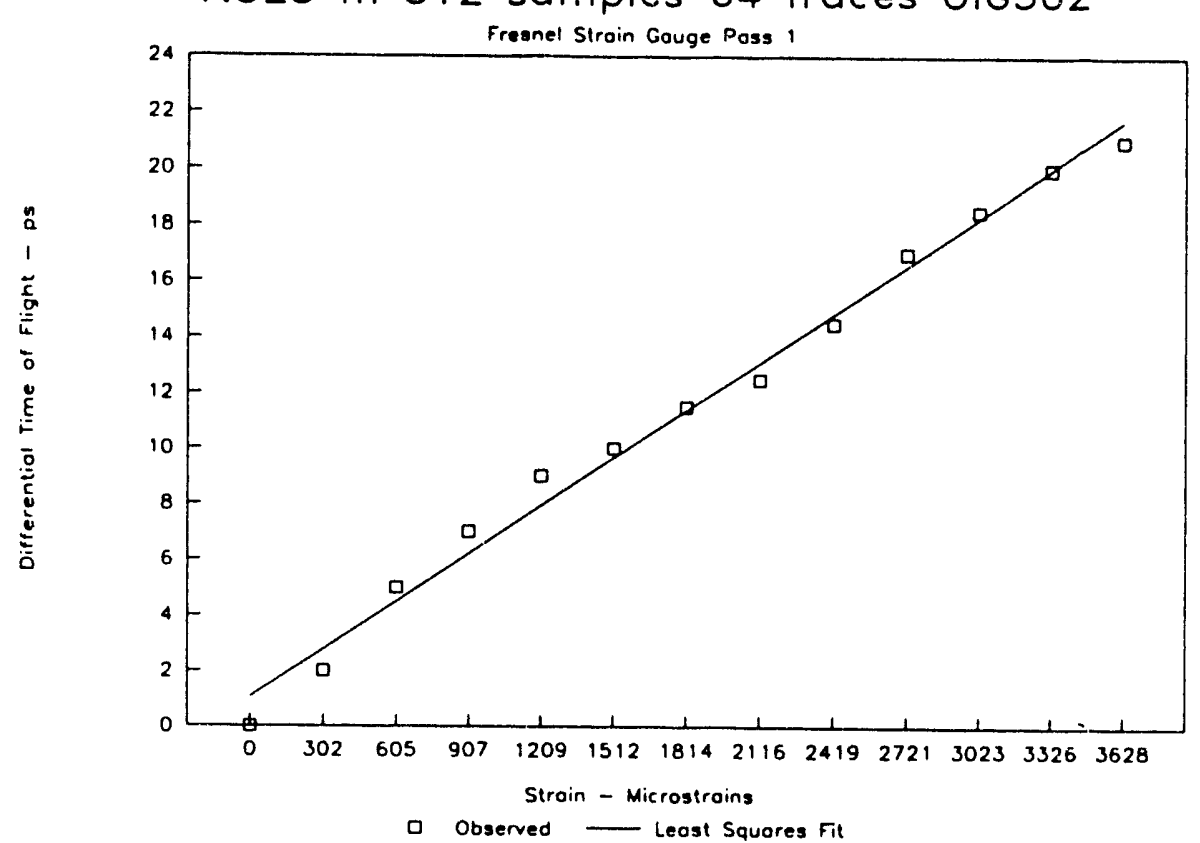

Fig. 30. Experiment 3, run 1.

\section{$1.323 \mathrm{~m} 512$ samples 64 traces 016502}

Fresnel Strain Gouge Poss 2

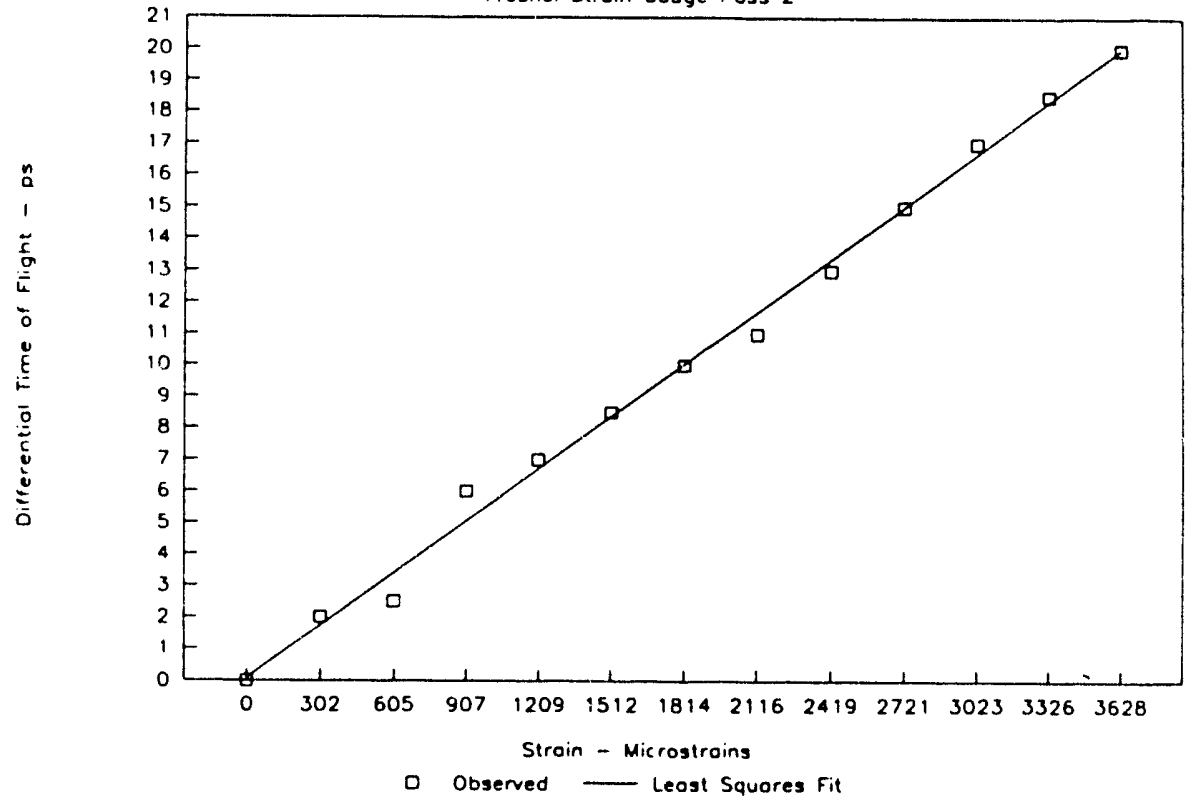

Fig. 31. Experiment 3, run 2. 
$1.323 \mathrm{~m} 512$ samples 64 traces $01 G 502$

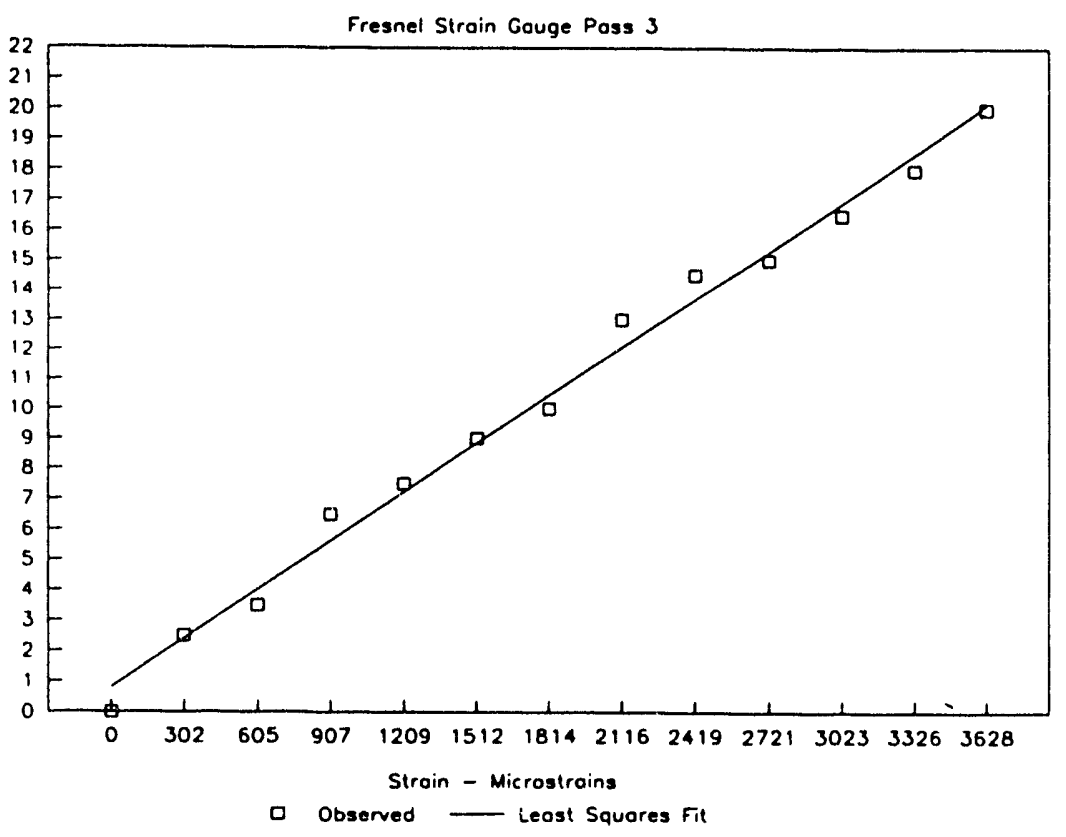

Fig. 32 Experiment 3, run 3.

\section{$1.323 \mathrm{~m} 512$ samples 64 traces $01 G 502$}

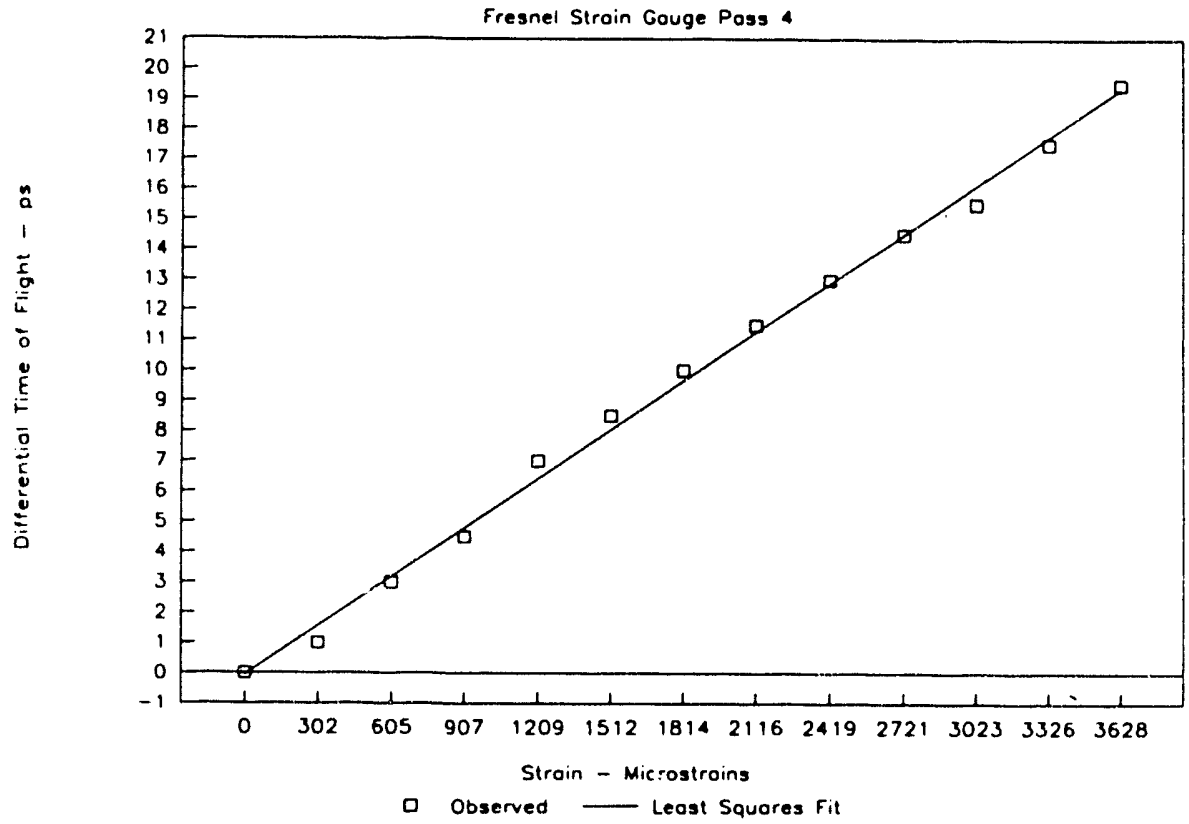

Fig. 33. Experiment 3, run 4. 
$1.323 \mathrm{~m} 512$ samples 64 traces 016502

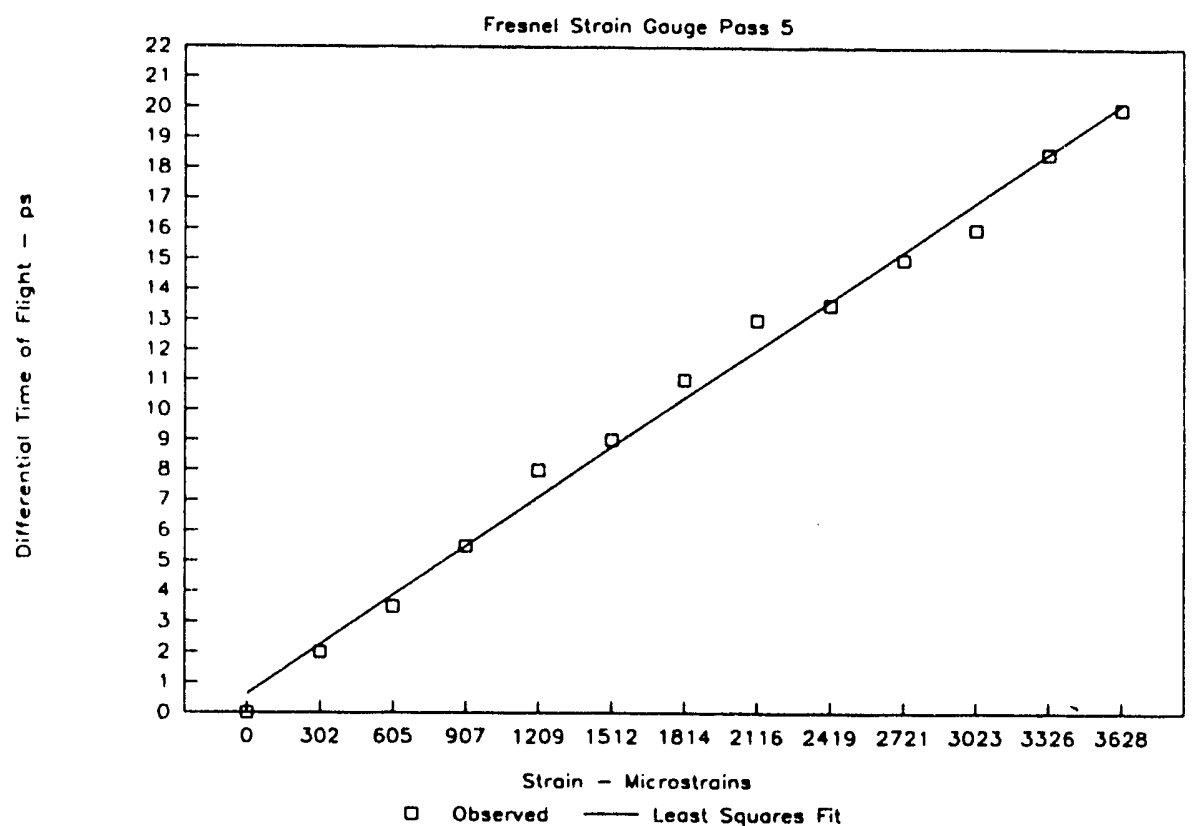

Fig. 34. Experiment 3, run 5.

\section{$1.323 \mathrm{~m} 512$ samples 64 traces 016502}

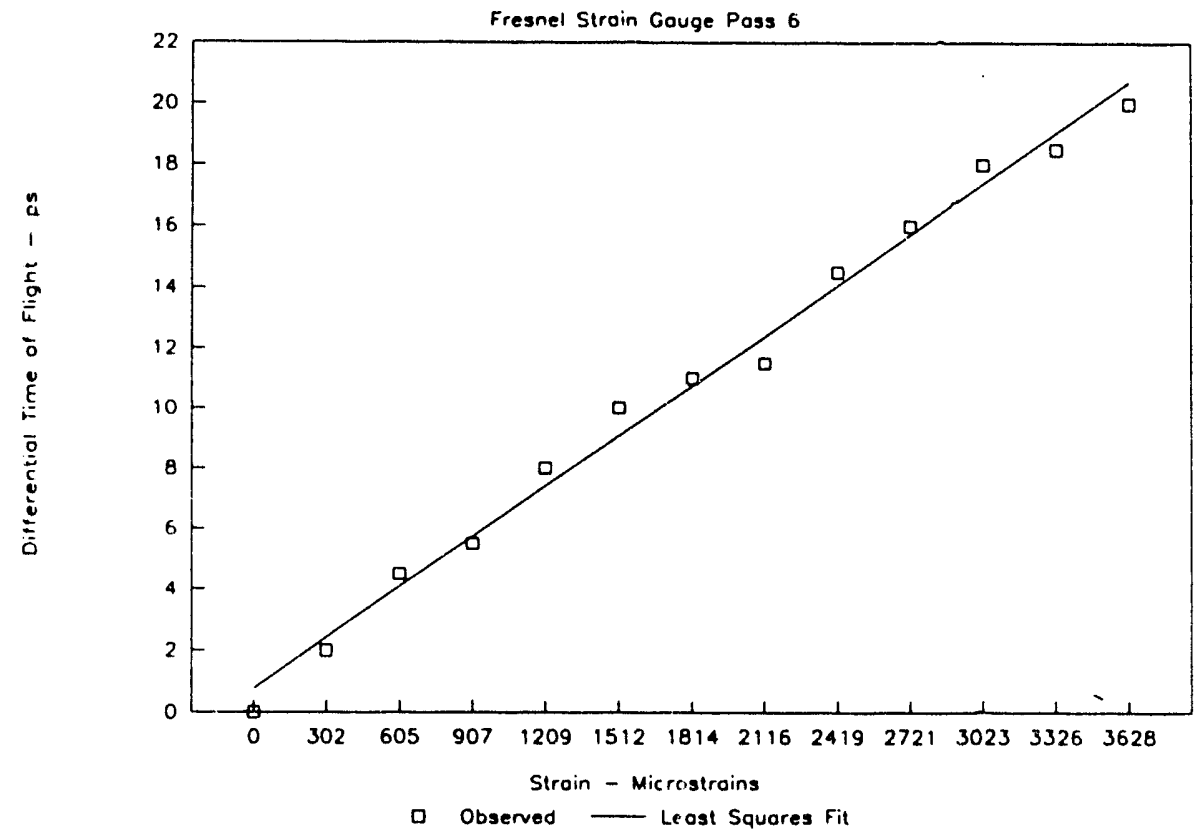

Fig. 35. Experiment 3, run 6 . 
standard deviation in error is $0.30 \mathrm{ps,} \mathrm{and} \mathrm{the} \mathrm{maximum} \mathrm{observed} \mathrm{error} \mathrm{is} 1.06 \mathrm{ps}$. If there is 99\% probability of being within three standard deviations of the mean, then, for these data, the error in the data has $99 \%$ probability of being within $\pm(0.43+3 \times 0.30)= \pm 1.33$ ps. This is well within the \pm 2 ps of uncertainty suggested by the data in Table 2 .

The fourth set of time-of-flight vs strain observations was made under exactly the same conditions as the second set, except that a different gauge fiber was used. The fourth set was made with the system using the PPL30K generator. The scope traces were made up of 512 samples, and the average of 32 traces was displayed. The fiber span was continuously supported to eliminate catenary effects. As the micrometer is adjusted to lower values, the micrometer screw pushes the stage in the direction that increases tension on the fiber. Thus, the imposed strain moves in the direction opposite the micrometer reading. For the experiment, a micrometer setting of $49.00 \mathrm{~mm}$ was found to be the point at which the fiber became taut and was taken as the zero strain point. The micrometer setting was decreased in increments of $400 \mu \mathrm{m}$ through an elongation range of 0 to $5000 \mu \mathrm{m}$, and the time of flight between peaks was observed at each micrometer setting.

Elongation is the difference between the zero strain micrometer setting of $49.00 \mathrm{~mm}$ and the actual micrometer setting. Strain in pure numbers is the elongation in meters divided by the measured gauge length of $1.2975 \mathrm{~m}$. Strain in microstrains is obtained by multiplying the pure number value of strain by $10^{6}$.

Differential time of flight is the difference in the time of flight observed at a given micrometer setting and the time of flight observed at the zero strain setting. Note that the time of flight through the gauge is half the time difference in the two peaks in the scope trace. The reason for this is that, in a reflective setup, the scope trace indicates how long the pulse takes to make two transits of the gauge fiber. Two sets of data are summarized in Table 6. For each set of time-of-flight vs applied strain data, the corresponding least squares straight line was computed. Both sets of observed data, and their least squares lines, are plotted in Fig. 36. The observed data points and the least squares lines for each of the two sets of observations are plotted in Figs. 37 and 38.

Some generalizations about these data can be drawn by considering the error statistics. For this purpose, the least squares line is assumed to be more accurate than the individual observations as a representation of the actual time-of-flight vs strain relationship. Error is defined as the absolute difference between the observed time of flight and the corresponding least squares time of night. For the data set in Table 6 the average error is $0.36 \mathrm{ps,} \mathrm{the}$ standard deviation in error is $0.24 \mathrm{ps}$, and the maximum observed error is $0.88 \mathrm{ps}$. If there is 99\% probability of being within three standard deviations of the mean, then, for these data, the error in the data has $99 \%$ probability of being within $\pm(0.36+3 \times 0.24)= \pm 1.08$ ps. This is well within the \pm 2 ps of uncertainty suggested by the data in Table 2 . Compare these error statistics with those for the second set of data, for which the average error is $0.44 \mathrm{ps}$, the standard deviation in error is $0.31 \mathrm{ps,} \mathrm{and} \mathrm{the} \mathrm{maximum} \mathrm{observed} \mathrm{error} \mathrm{is} 1.49 \mathrm{ps}$.

The experimental setup for the fifth set of time-of-flight vs strain observations is the loop scheme shown in Figs. 3 and 4. The generator is the Opto-Electronics PPL30K. The resulting trace is illustrated in Fig. 39, the scope trace of December 6, 1991, at 16:36:40. A second trace is generated from the main trace by adding a small constant. A window is generated from each trace, allowing the spikes at both ends of the trace to be viewed simultaneously. The time base of the windows is expanded to allow for the precise location of the peaks. The total time difference is found by using a split cursor, one in each window; placing the cursors at the peaks; and letting the scope calculate the time difference between them. The five spikes visible in the main trace represent four transits through the strain gauge loop by the OTDR pulse. 
Table 6. Experiment 4-Time of flight us applied strain

(PPL30K generator; 512 samples, 32 traces;

gauge continuously supported; 1.2975 -m gauge length; Fresnel reflection configuration)

\begin{tabular}{cccccc}
\hline & \multicolumn{3}{c}{ Time of flight: one transit through gauge (ps) } \\
\cline { 2 - 3 } $\begin{array}{c}\text { Strain } \\
\text { (microstrains) }\end{array}$ & \multicolumn{2}{c}{ Observed } & & \multicolumn{2}{c}{ Least squares } \\
\cline { 2 - 3 } \cline { 5 - 6 } & Run 1 & Run 2 & & Run 1 & Run 2 \\
\hline 0 & 0 & 0 & & -0.15398 & 0.351470 \\
308 & 1.5 & 1.5 & & 1.287025 & 1.778764 \\
617 & 2.5 & 3.5 & & 2.732715 & 3.210692 \\
925 & 4 & 5.5 & & 4.173727 & 4.637986 \\
1233 & 6 & 6.5 & & 5.614738 & 6.065280 \\
1541 & 7.5 & 7.5 & & 7.055749 & 7.492574 \\
1850 & 8.5 & 8.5 & & 8.501439 & 8.924502 \\
2158 & 9.5 & 10 & & 9.942450 & 10.35179 \\
2466 & 10.5 & 11.5 & & 11.38346 & 11.77909 \\
2775 & 12.5 & 12.5 & & 12.82915 & 13.21101 \\
3083 & 14.5 & 15 & & 14.27016 & 14.63831 \\
3391 & 15.5 & 16 & & 15.71117 & 16.06560 \\
3699 & 18 & 18 & & 17.15218 & 17.49290 \\
\hline
\end{tabular}

The fifth set of time-of-flight vs strain observations was made with the system using the PPL30K generator. The scope traces were made up of 512 samples, and the average of 32 traces was displayed. The fiber span was continuously supported to eliminate catenary effects. As the micrometer is adjusted to lower values, the micrometer screw pushes the stage in the direction that increases tension on the fiber. Thus, the imposed strain moves in the direction opposite the micrometer reading. For the experiment, a micrometer setting of $48.00 \mathrm{~mm}$ was found to be the point at which the fiber became taut and was taken as the zero strain point. The micrometer setting was decreased in increments of $400 \mu \mathrm{m}$ through an elongation range of 0 to $5000 \mu \mathrm{m}$, and the time of flight between peaks was observed at each micrometer setting.

Elongation is the difference between the zero strain micrometer setting of $48.00 \mathrm{~mm}$ and the actual micrometer setting. Strain in pure numbers is the elongation in meters divided by the measured gauge length of $1.2975 \mathrm{~m}$. Strain in microstrains is obtained by multiplying the pure number value of strain by $10^{6}$.

Differential time of flight is the difference in the time of flight observed at a given micrometer setting and the time of flight obser 'ed at the zero strain setting. Note that the time of flight through the gauge is one-fourth the time difference between the first and fifth peaks in the scope trace. The reason for this is that, in a loop setup, the scope trace indicates how long the pulse takes to make multiple transits of the gauge fiber. Six sets of data are summarized in Table 7. For each set of time-of-flight vs applied strain data, the corresponding least squares straight line was computed. All six sets of observed data are plotted in Fig. 40. The six least squares lines are plotted in Fig. 41. The observed data points and the least squares lines for each of the four sets of observations are plotted in Figs. 42 to 47. 


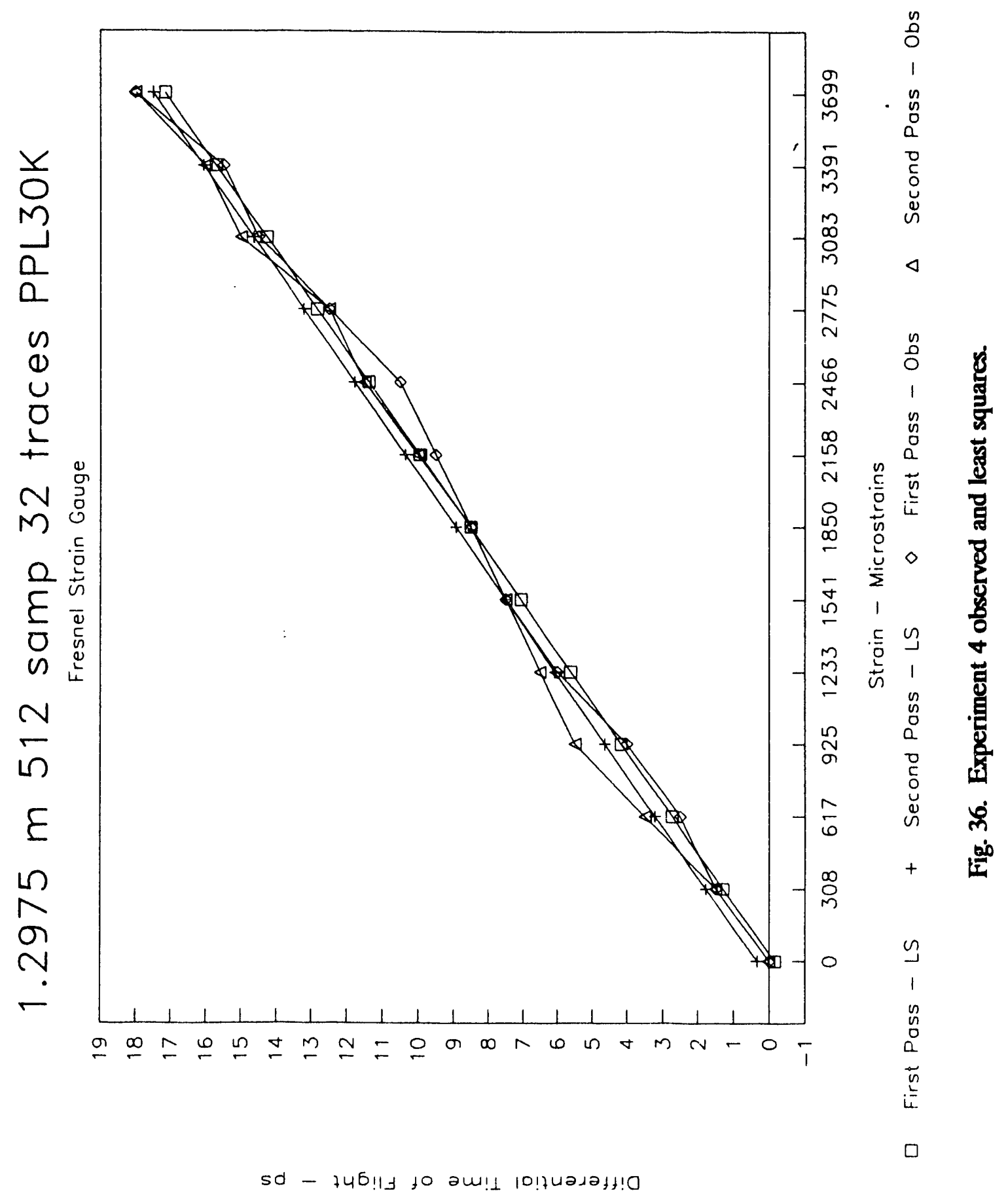


$1.2975 \mathrm{~m} 512$ samp 32 traces PPL3OK

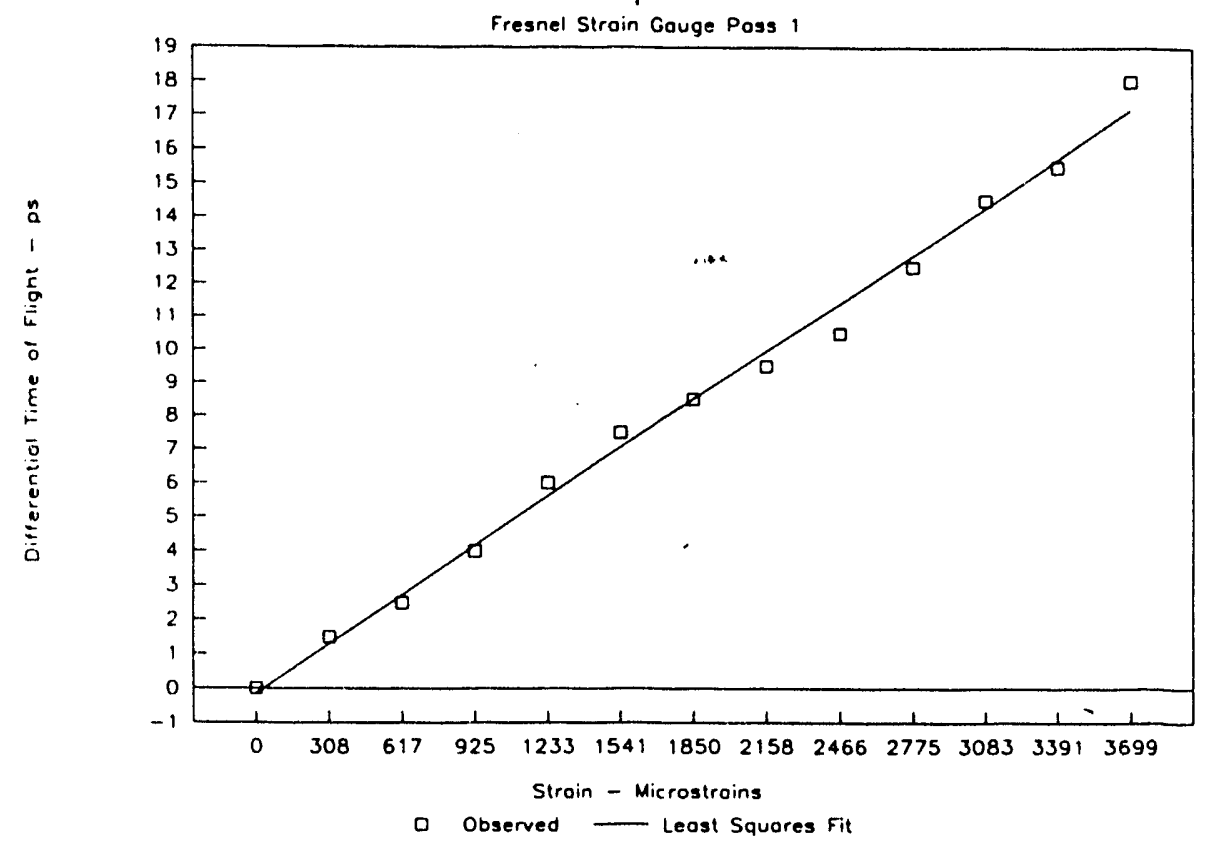

Fig. 37. Experiment 4, run 1.

$1.2975 \mathrm{~m} 512$ samp 32 traces PPL3OK Fresnel Strain Gauge Pass 2

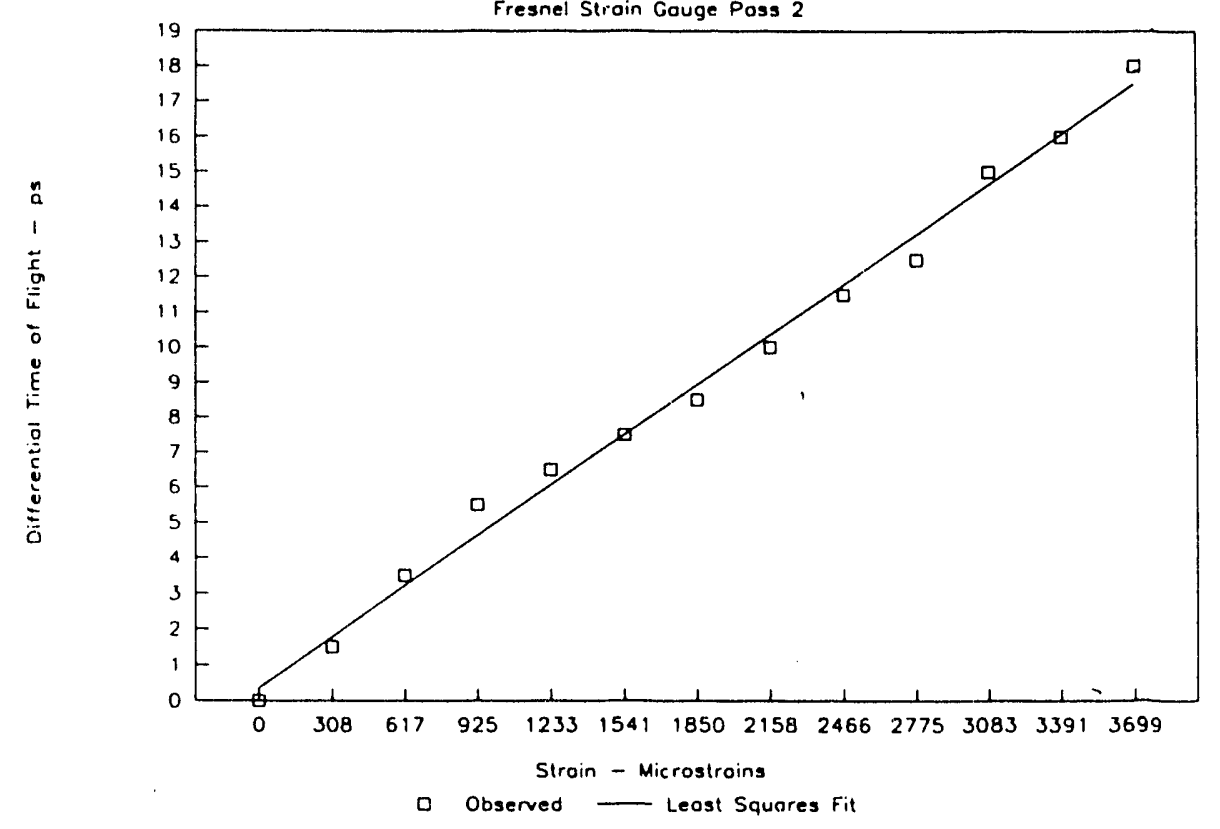

Fig. 38. Experiment 4, run 2. 

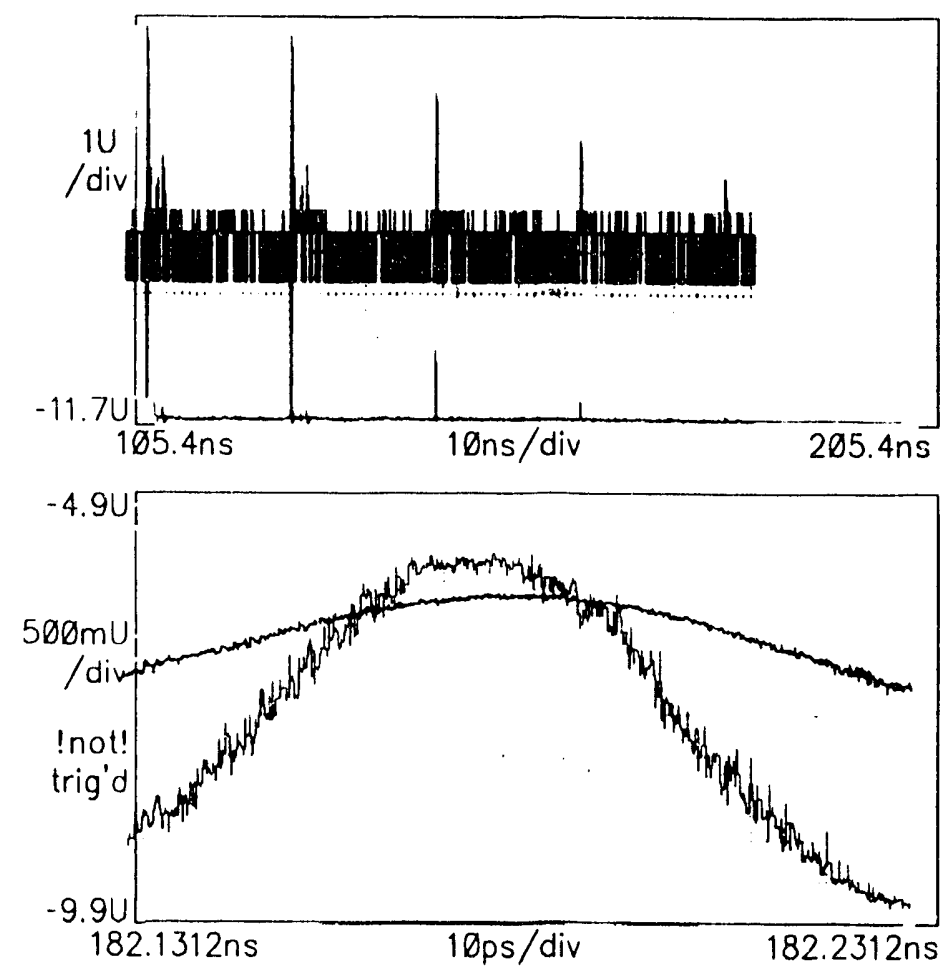

\begin{tabular}{|c|c|c|c|c|}
\hline $\begin{array}{c}\text { Vertical } \\
\text { Desc }\end{array}$ & $\begin{array}{c}\text { Horizontal } \\
\text { Desc }\end{array}$ & $\begin{array}{c}\text { Acquire } \\
\text { Desc }\end{array}$ & $\begin{array}{l}\text { Lower } \\
\text { Graticule }\end{array}$ & $\frac{\text { Average }}{32}$ \\
\hline \multirow{2}{*}{$\begin{array}{l}\text { Avg }(\operatorname{Ln}(\mathrm{M} 4 . \\
\text { High Prec }\end{array}$} & \multirow{2}{*}{$\begin{array}{l}\text { Window } \\
\text { (\$) } 512 \text { pts }\end{array}$} & \multirow[t]{2}{*}{ Stopped } & \multirow{2}{*}{$\begin{array}{c}\text { Dual } \\
s, v\end{array}$} & Average $N$ \\
\hline & & & & 32 \\
\hline \multirow[t]{2}{*}{$\begin{array}{l}\text { Sampling } \\
\text { Head Fnc's } \\
\end{array}$} & \multirow{2}{*}{$\begin{array}{l}\text { Window } \\
\text { Mode } \\
\text { Manual }\end{array}$} & $\begin{array}{c}\text { Save Trace } \\
\text { Desc }\end{array}$ & More... & $\begin{array}{l}\text { Remove } / \mathrm{Cl} \\
\text { Trace } 4\end{array}$ \\
\hline & & & $\begin{array}{c}\text { Trace } \\
\text { Status }\end{array}$ & $\begin{array}{c}\text { Avg }(\operatorname{Ln}(M 4 . \\
\text { Window }\end{array}$ \\
\hline
\end{tabular}

Fig. 39. Display for loop OTDR. 


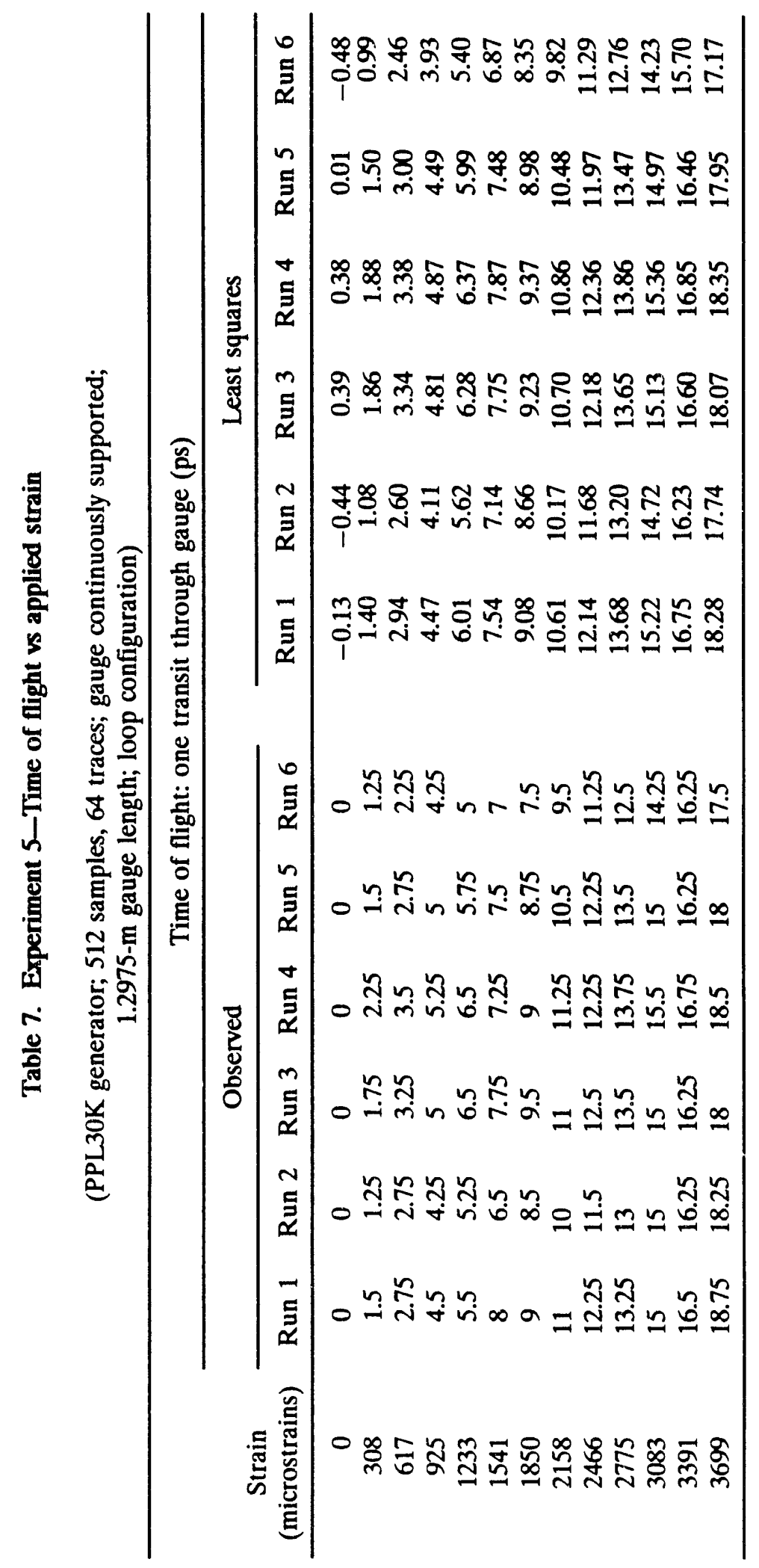




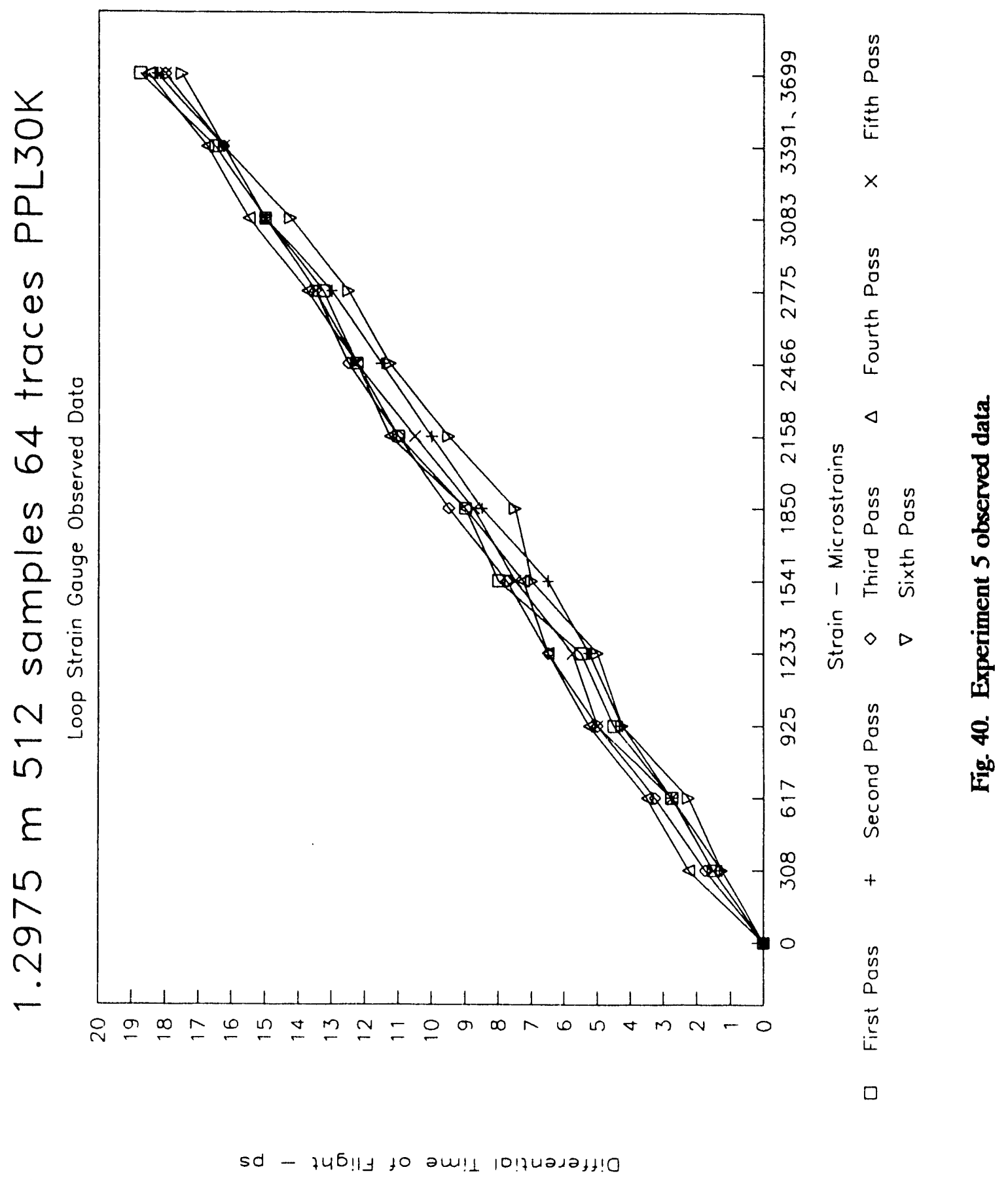




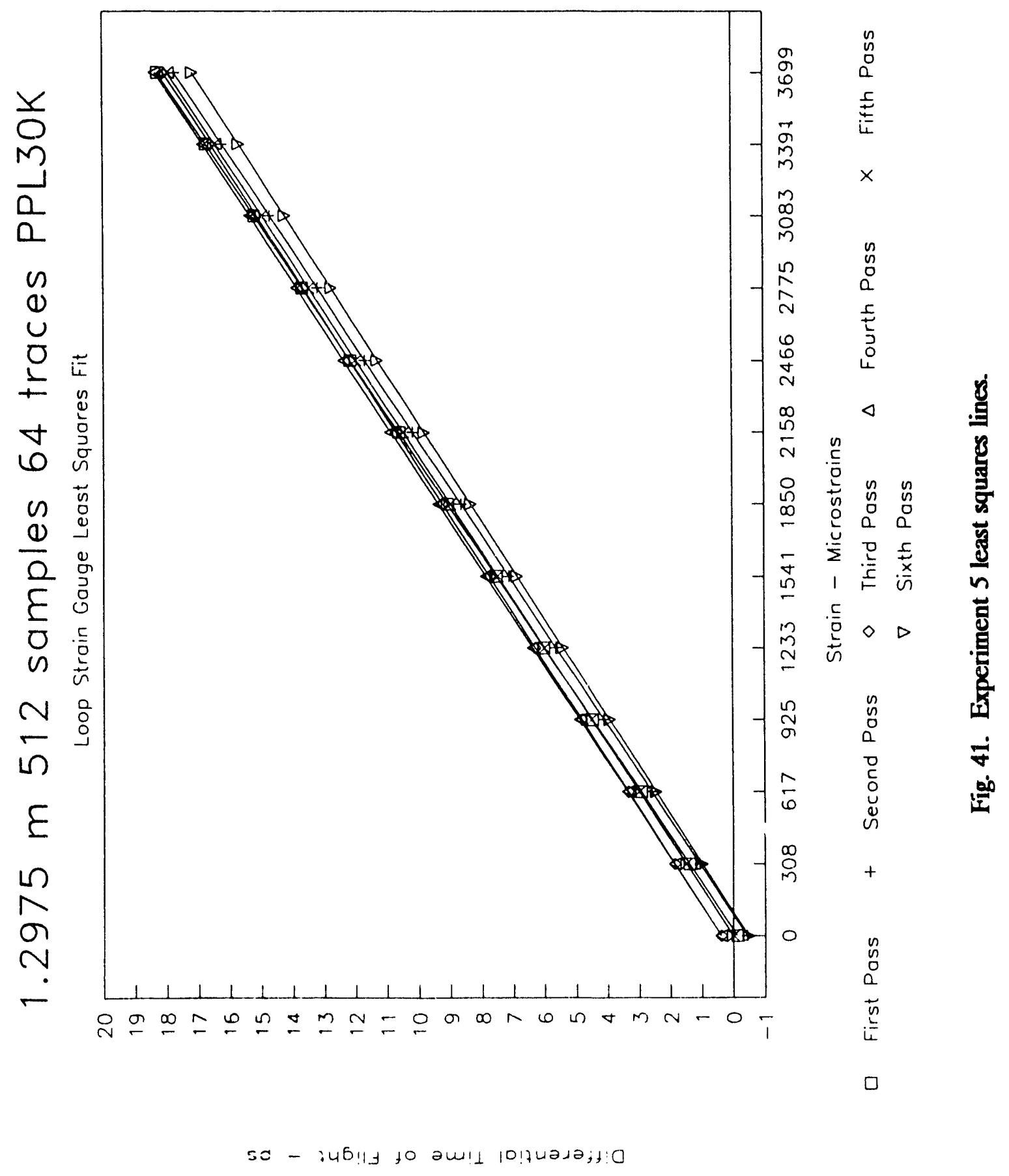




\section{$1.2975 \mathrm{~m} 512$ samples 64 traces PPL3OK}

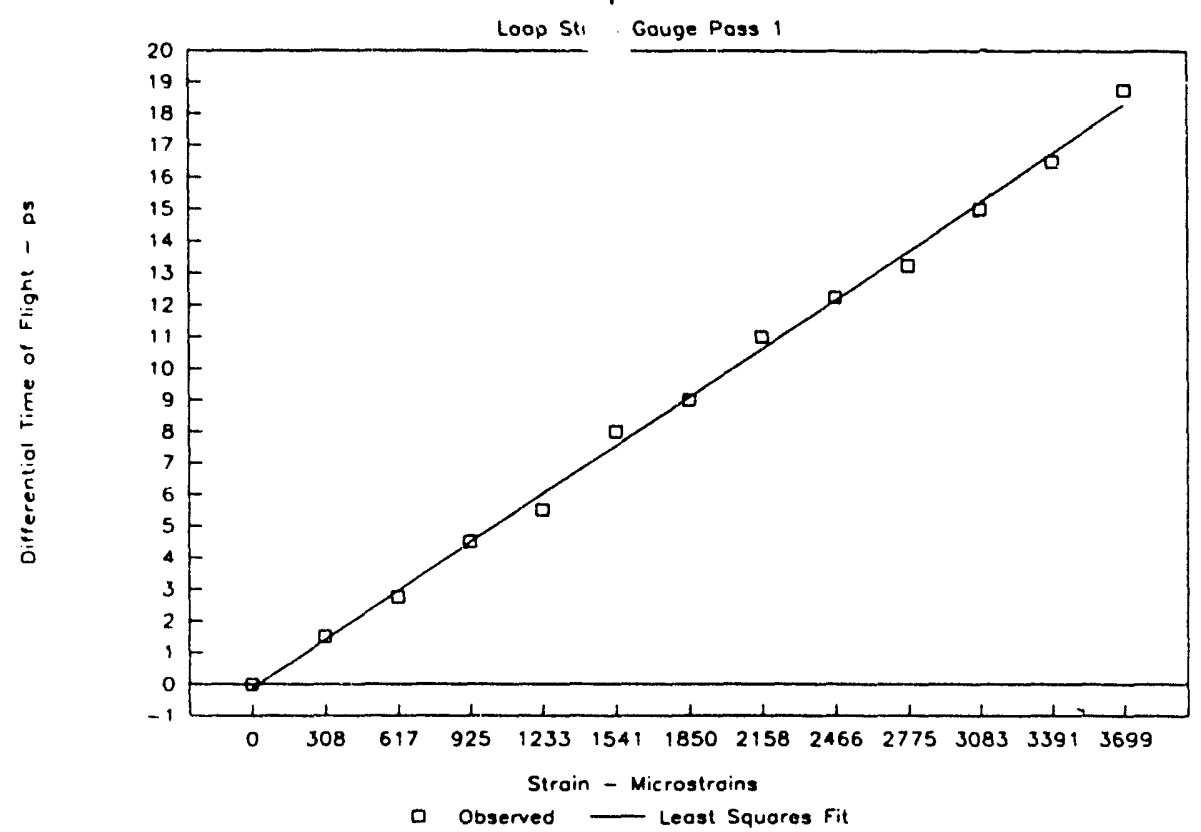

Fig. 42 Experiment 5, run 1.

\section{$1.2975 \mathrm{~m} 512$ samples 64 traces PPL3OK}

Loop Stroin Gouge Pass 2

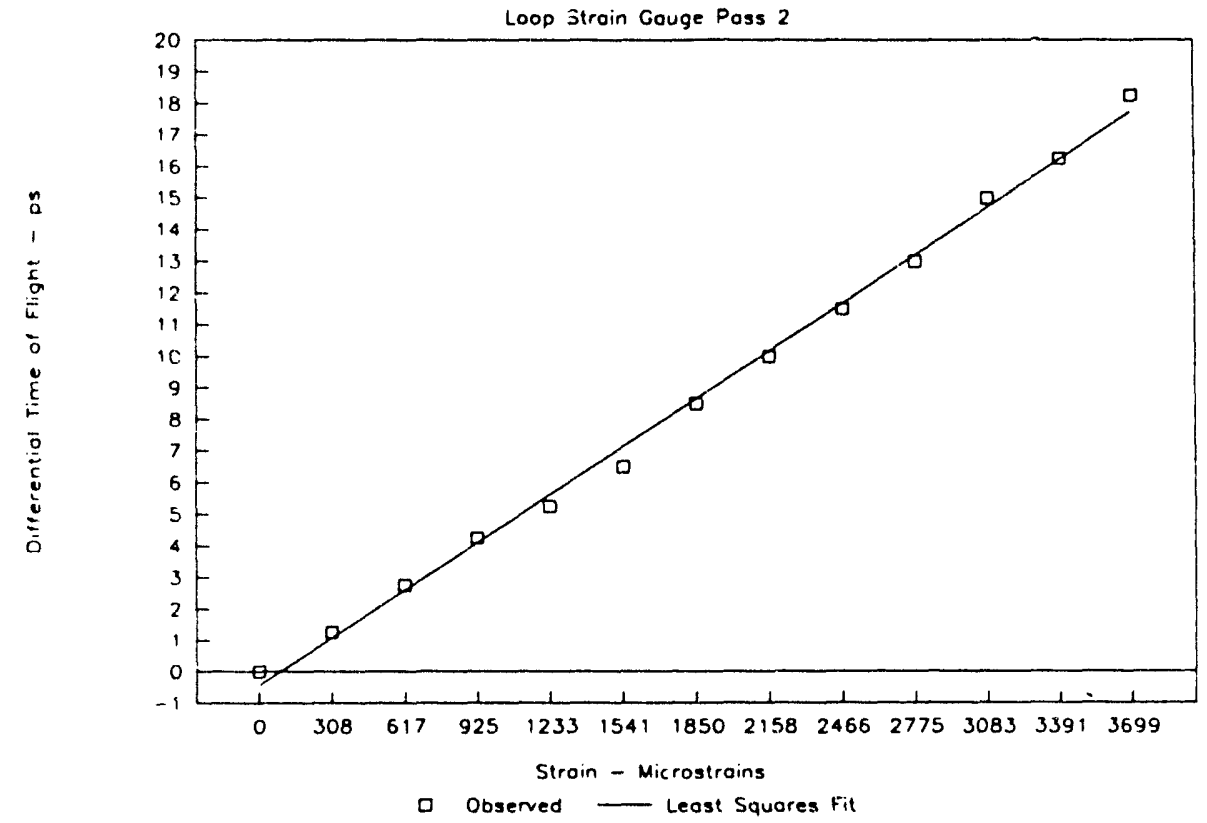

Fig. 43. Expcriment 5, run 2 
$1.2975 \mathrm{~m} 512$ samples 64 traces PPL3OK

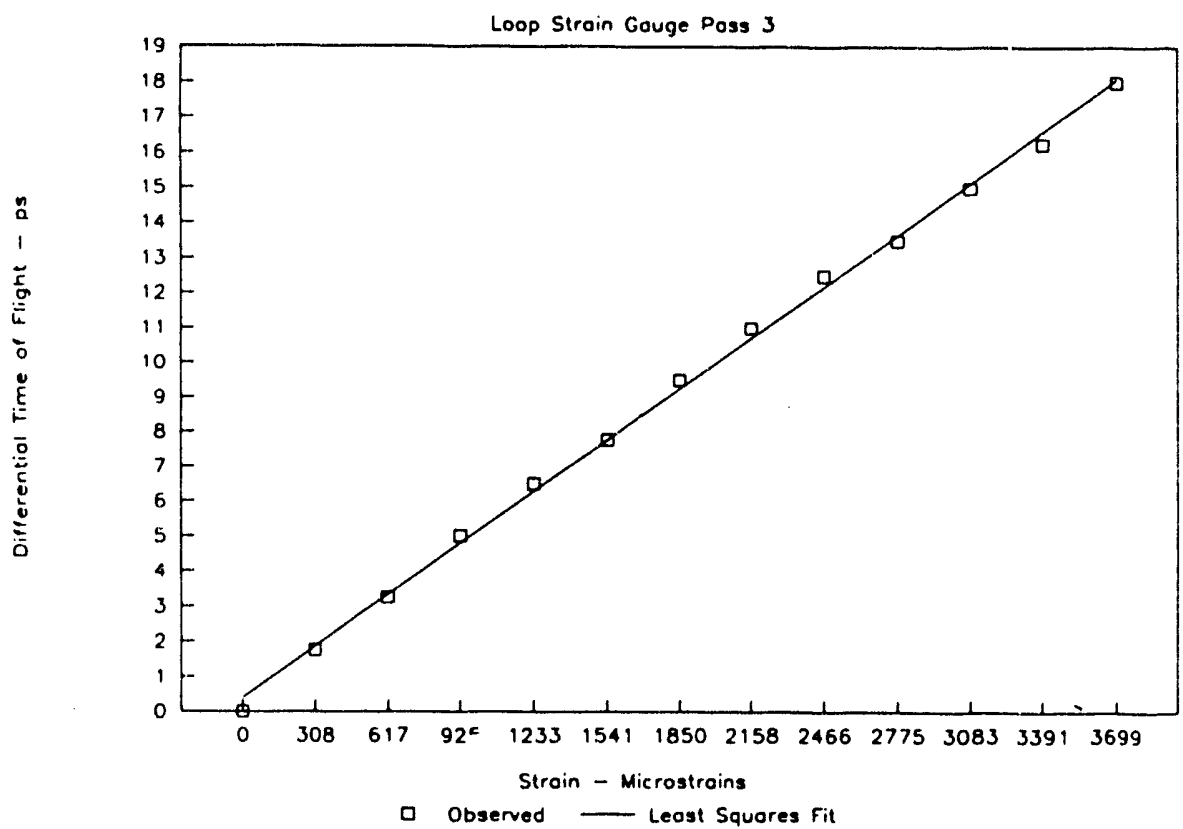

Fig. 44. Experiment 5, run 3.

$1.2975 \mathrm{~m} 512$ samples 64 traces PPL3OK Loop Stroin Gouge Pass A

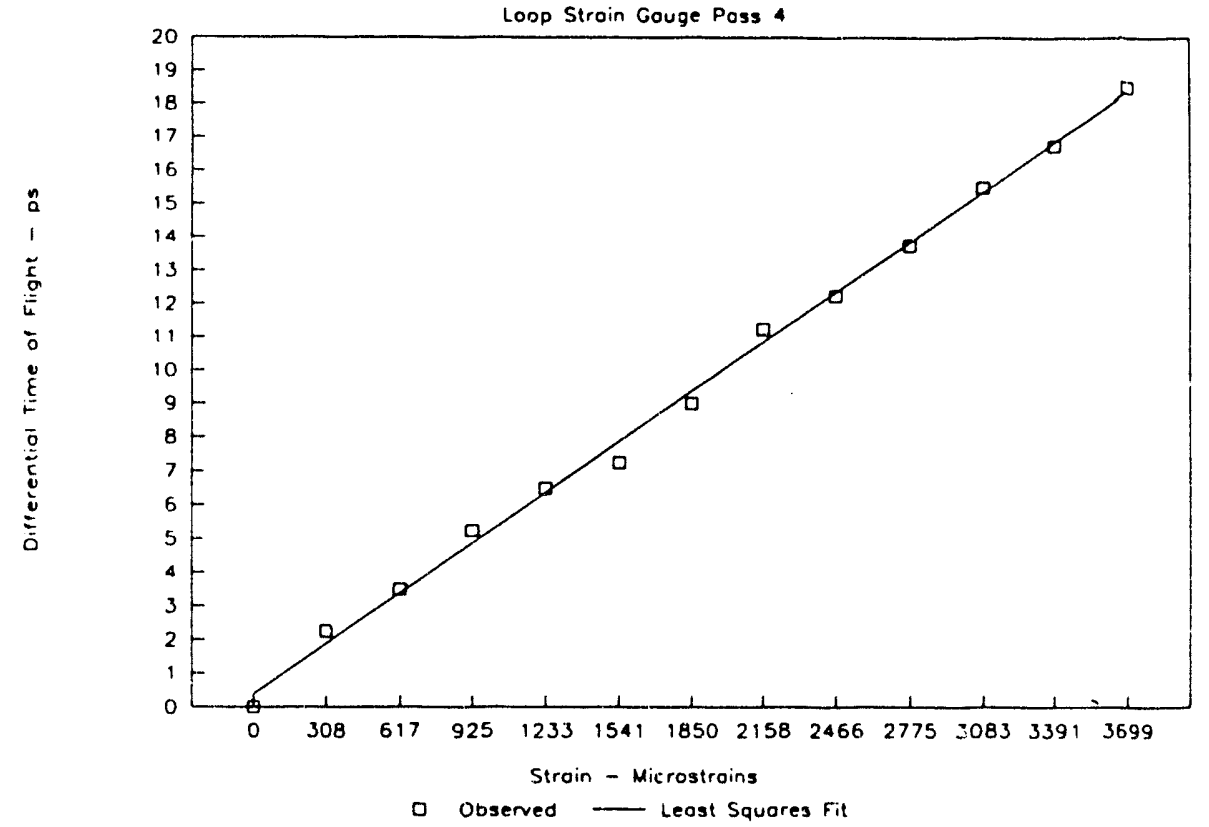

Fig. 45. Experiment 5, run 4. 

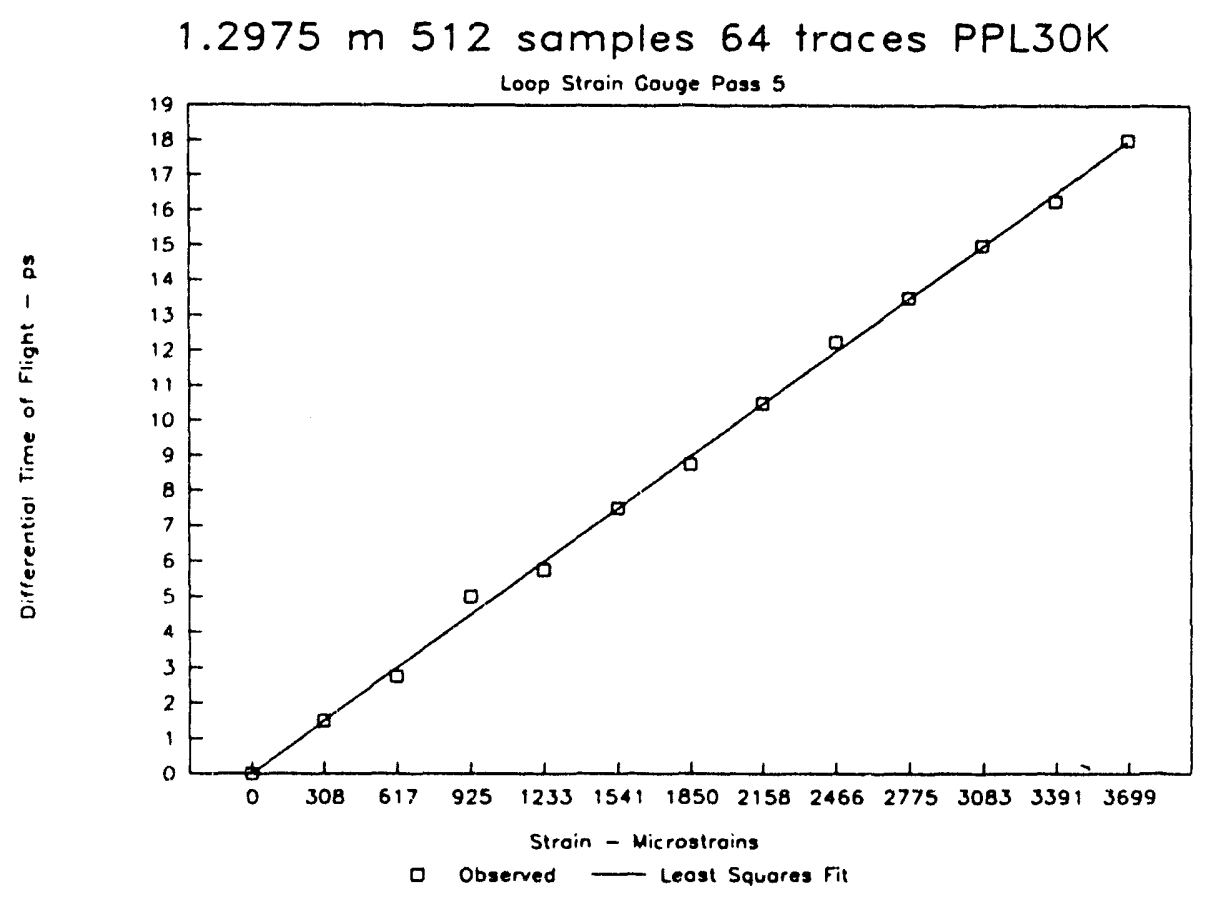

Fig. 46. Experiment 5, run 5.

\section{$1.2975 \mathrm{~m} 512$ samples 64 traces PPL3OK} Loop Strain Gouge Pass 6

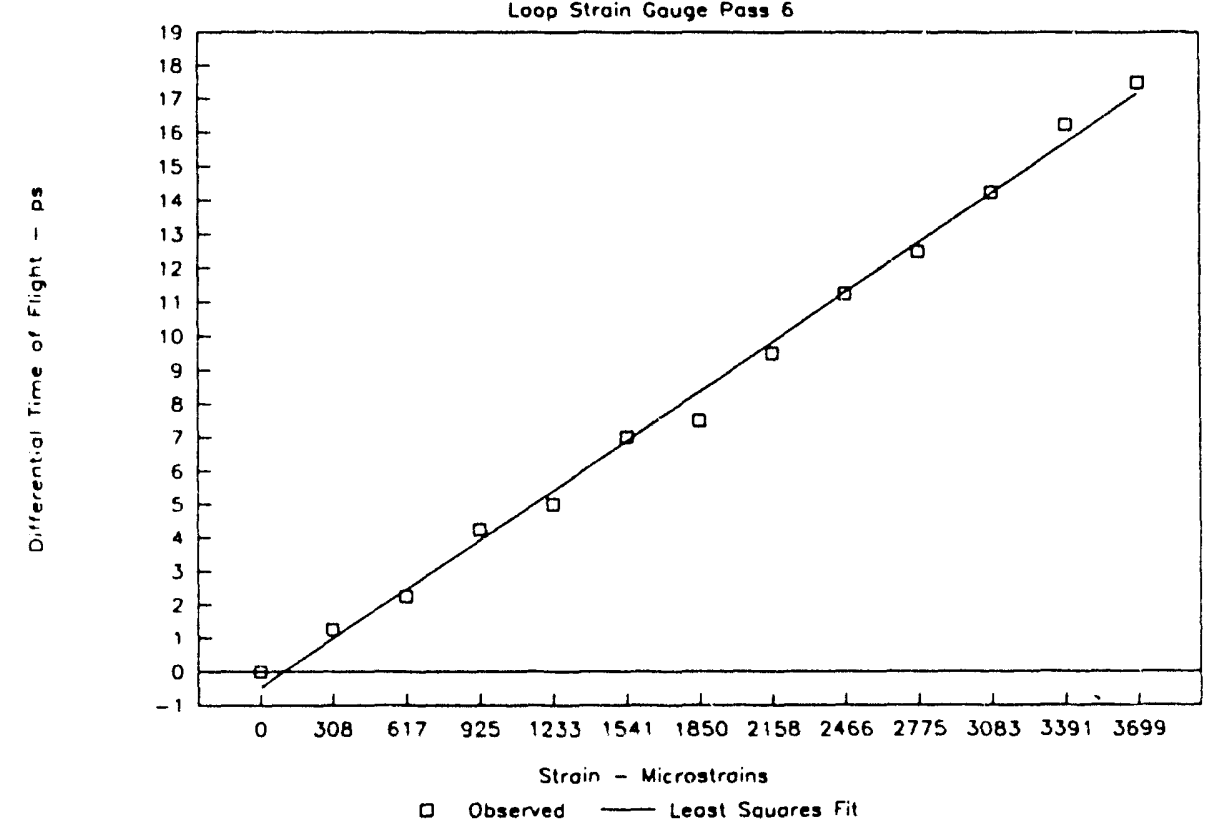

Fig. 47. Experiment 5, run 6. 
Some generalizations about these data can be drawn by considering the error statistics. For this purpose, the least squares line is assumed to be more accurate than the individual observations as a representation of the actual time-of-flight vs strain relationship. Error is defined as the absolute difference between the observed time of flight and the corresponding least squares time of flight. For the data set in Table 7 the average error is $0.27 \mathrm{ps,}$, the standard deviation in error is $0.17 \mathrm{ps}$, and the maximum observed error is $0.84 \mathrm{ps}$. If there is 99\% probability of being within three standard deviations of the mean, then, for these data, the error in the data has $99 \%$ probability of being within $\pm(0.27+3 \times 0.17)= \pm 0.78$ ps. This is well within the \pm 1 -ps uncertainty expected by taking a reflective system in which the OTDR pulse makes two passes through the gauge and leads to \pm 2 -ps uncertainty and modifying it to double its performance by using a loop scheme in which the OTDR pulse makes four passes through the gauge.

For the five strain experiments, the error statistics are summarized in Table 8, and plotted in Figs. 48 and 49 . What these data show is that the experimental procedure is highly repeatable. The first four experiments show that, for the strain gauge based on Fresnel reflection, in which the OTDR pulse passes through the gauge twice, the time of flight for one pass of the OTDR pulse through the gauge fiber is well within \pm 2 -ps uncertainty. The fifth experiment shows that, for the loop strain gauge, in which the OTDR pulse passes through the gauge four times, the time of flight for one pass of the OTDR pulse through the gauge fiber is well within \pm 1 -ps uncertainty. Figure 48 shows statistics for all five experiments. Figure 49 shows statistics for experiment 2 (Fresnel) and experiment 5 (loop) and gives an indication of the comparative performance of the two configurations.

Table 9 and Fig. 50 show repeatability data for different configurations and equipment settings. For each of these 14 repeatability experiments, the strain gauge was set up, and the strain micrometer was set to the zero strain position. Then the indicated number of observations of time of flight was made. The times are the time (in picoseconds) for the OTDR pulse to make one pass through the gauge fiber. For each set of observations, the standard deviation, in picoseconds, was computed.

Several conclusions can be drawn from the repeatability data. The PPL30K laser produces less variation in the time-of-flight readings than the 5-dB weaker OIG-502 laser. This is not surprising since it is easier to precisely locate the peak of the spike of the more powerful signal. Traces based on the average of 64 scans tended to produce less variation than traces with more or fewer scans. The idea that there should be an optimal averaging rate is not surprising. Increasing the number of scans averaged in the trace produces two conflicting effects. Noise tends to be averaged out, making the peak of the spike easier to locate, but time jitter causes the averaged peak of the spike to broaden, making it more difficult to precisely locate.

Making an observation of the length of an event of long duration should lead to very accurate measurement of the length of its shorter subevents. In a setup in which the OTDR pulse makes multiple transits through the gauge, the uncertainty in the time of flight for one transit is the total uncertainty in the observation divided by the number of gauge transits represented in the observation. In Fig. 48, in which strain is applied, the observations of change in time of flight for one transit through the gauge vs change in applied strain indicate that the loop, through which the OTDR pulse makes four transits, has about half as much measurement uncertainty as the Fresnel configuration, through which the OTDR pulse makes two transits.

John Marton of Opto-Electronics was kind enough to let us use an OFM 10 self-contained subpicosecond OTDR unit for 1 day. Several sets of observations of time of flight between Fresnel discontinuities vs applied strain were made with the unit. It was 


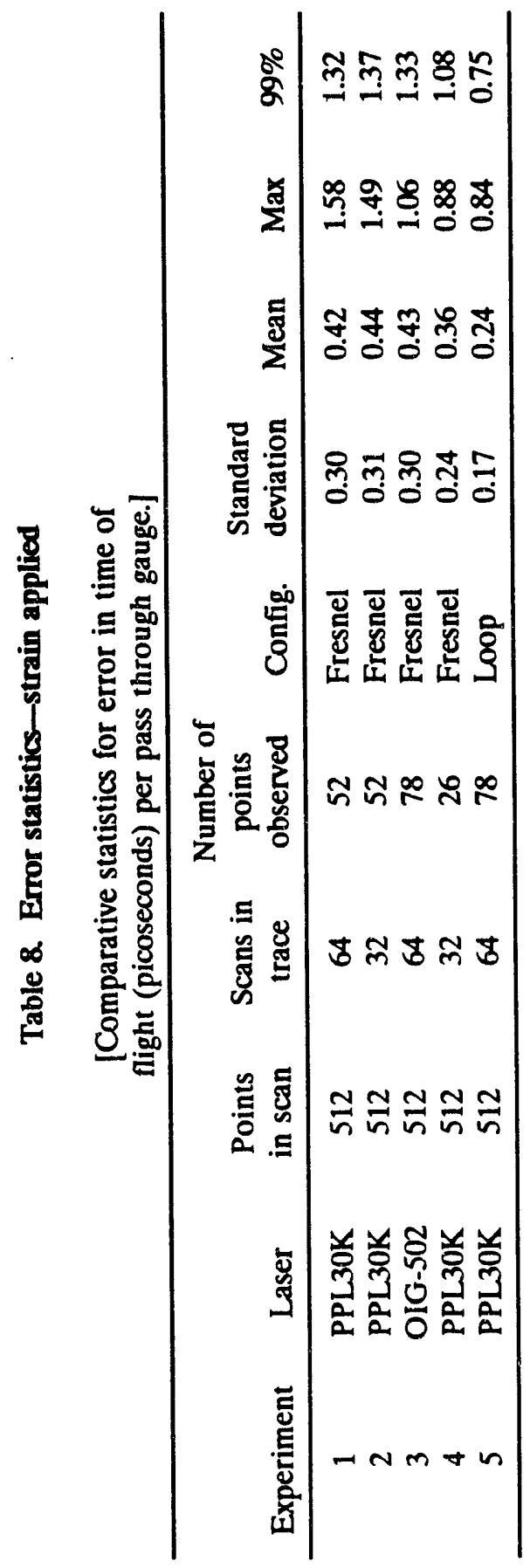




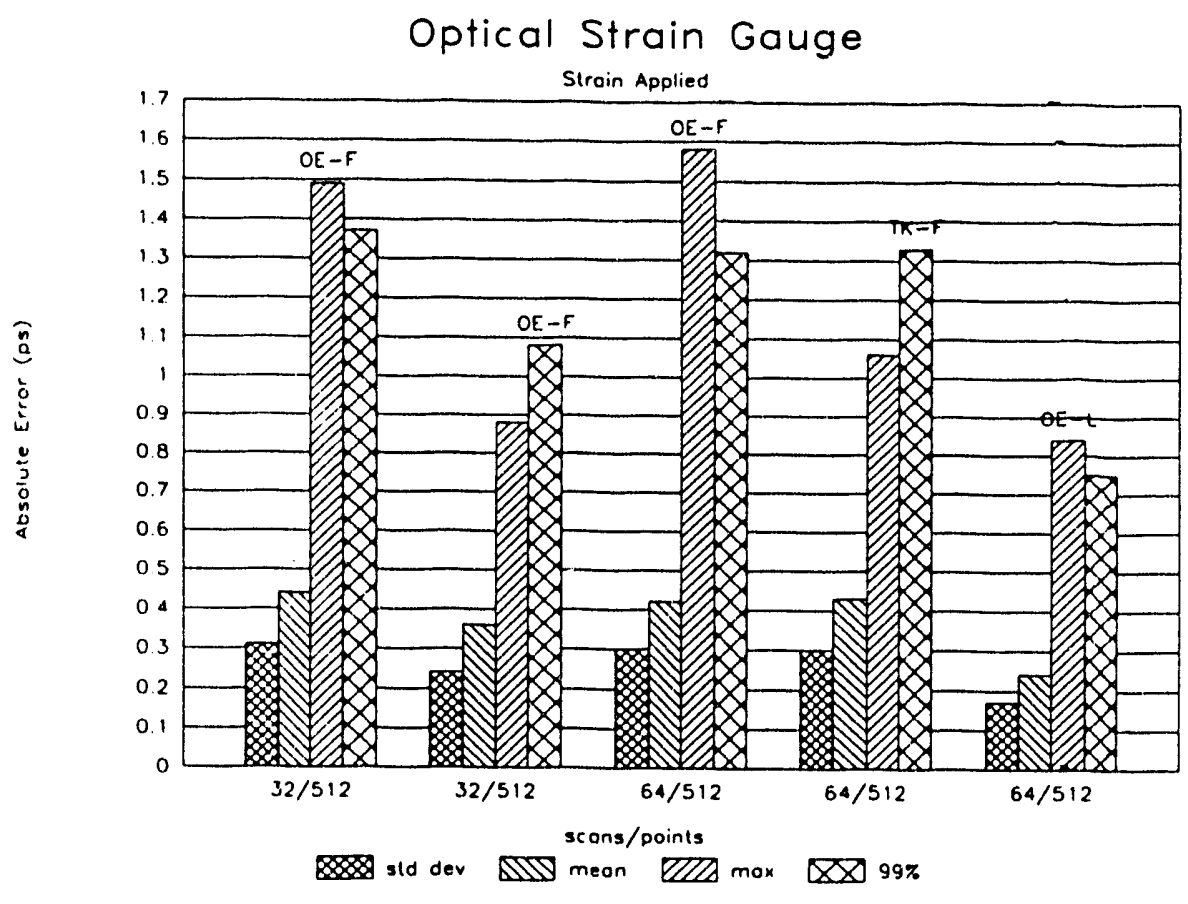

Fig. 48. Error statistics for experiments 1 to 5.

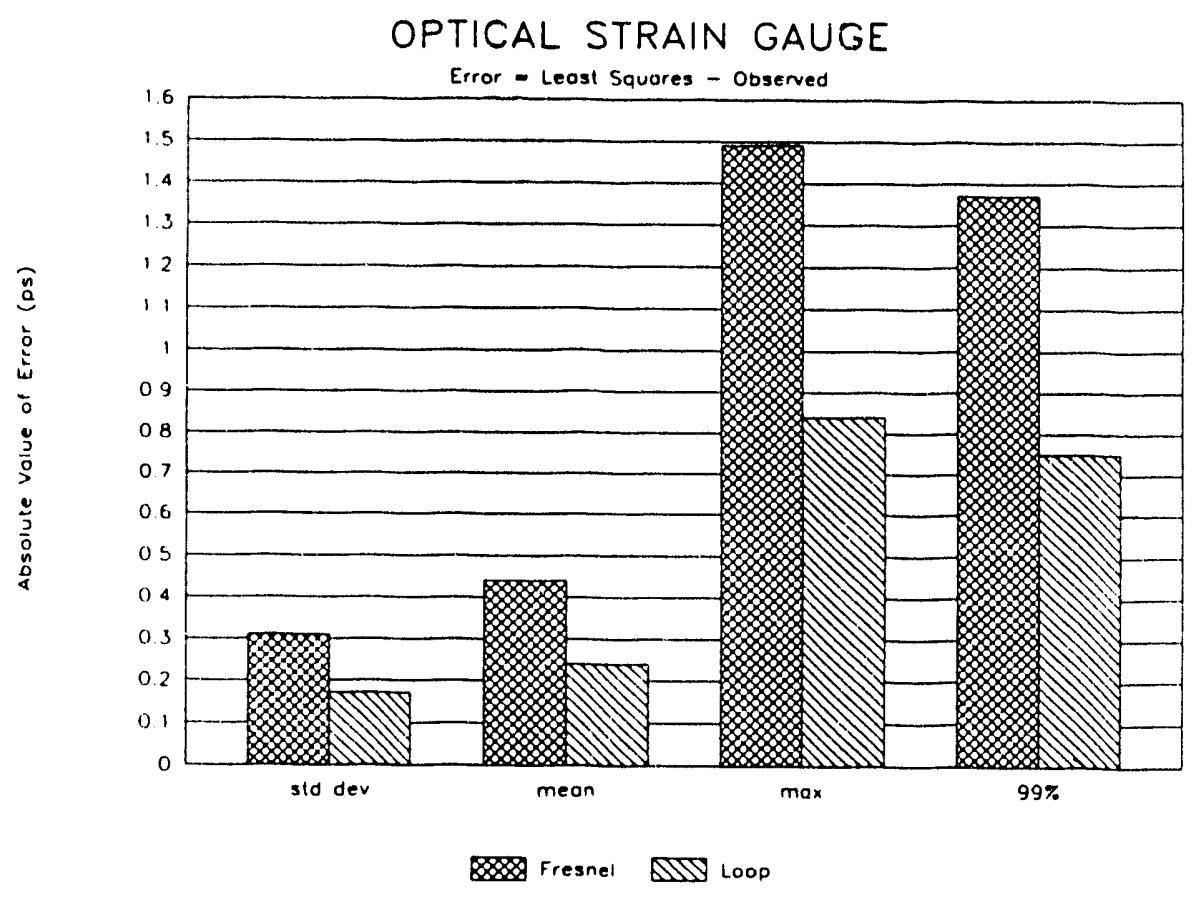

Fig. 49. Error statistics for experiments 2 and 5. 
Table 9. Error statistics-no strain applied

(Repeatability for various settings of strain gauge electronics. Standard deviation in readings of time of flight in picoseconds for one pass through gauge fiber.)

\begin{tabular}{ccccccc}
\hline Experiment & Laser & Points & Traces & Observations & Config. & $\begin{array}{c}\text { Standard } \\
\text { deviation }\end{array}$ \\
\hline 1 & PPL30K & 512 & 32 & 8 & Fresnel & 0.43 \\
2 & PPL30K & 512 & 64 & 8 & Fresnel & 0.35 \\
3 & PPL30K & 512 & 32 & 20 & Fresnel & 0.51 \\
4 & OIG-502 & 512 & 64 & 20 & Fresnel & 0.83 \\
5 & PPL30K & 512 & 32 & 4 & Fresnel & 0.56 \\
6 & OIG-502 & 512 & 256 & 4 & Fresnel & 1.7 \\
7 & OIG-502 & 512 & 64 & 4 & Fresnel & 1.23 \\
8 & OIG-502 & 512 & 256 & 4 & Fresnel & 1.98 \\
9 & OIG-502 & 512 & 64 & 4 & Fresnel & 2.56 \\
10 & PPL30K & 2048 & 128 & 4 & Loop & 0.99 \\
11 & PPL30K & 2048 & 64 & 4 & Loop & 0.35 \\
12 & PPL30K & 1024 & 128 & 4 & Loop & 0.82 \\
13 & PPL30K & 1024 & 64 & 4 & Loop & 0.37 \\
14 & PPL30K & 512 & 64 & 4 & Loop & 0.44 \\
\hline
\end{tabular}

operated under less than optimal conditions. It operates at $850 \mathrm{~nm}$, and these observations were done with $9-\mu \mathrm{m}$ fibers (single mode at $1300 \mathrm{~nm}$ ). This led to a much lower SNR (hence resolution) than would have been possible had single-mode, $850-\mathrm{nm}$ fibers been available. Also, the internal display of the OTDR unit has \pm 1 -ps uncertainty in the output reading, even though the uncertainty in the underlying electronics is $\pm 0.5 \mathrm{ps}$. Using a computer interface to read the underlying data would have led to more precise results than those obtained from the OTDR display.

It is also noted that a slightly different experimental procedure was used with the OFM 10. For the experiments previously described, the time-of-flight vs strain readings were taken for individual settings of the micrometer. A single set of readings would be taken as the fiber was stretched, and another set would be taken as it was relaxed. Thus, the four curves taken in Fig. 16 represent two successive stretches and relaxations of the gauge. For the experiments made with the OFM 10, multiple readings were taken on a single stretch of the gauge fiber.

The data shown in Table 10 and Fig. 51 were taken with the same gauge fiber as was used to collect the data in Tables 3,4 , and 5 . After a strain of less than $3 \mathrm{~mm}$, the epoxy bonding the gauge fiber to its connector failed. These data are presented to indicate the erratic behavior that occurs as the gauge fiber is about to fail.

The data shown in Table 11 and Fig. 52 were taken with a different gauge fiber epoxied directly to the mounting hardware. Note that these data are absolute time of flight versus micrometer setting, as contrasted with the differential time of flight vs applied strain, as shown in Tables 3 to 7. Error is defined as the absolute difference between the observed time of flight and the corresponding least squares time of flight. For the data set in Table 11 the average error is $0.69 \mathrm{ps}$, the standard deviation in error is $0.55 \mathrm{ps}$, and the maximum observed error is $2.42 \mathrm{ps}$. If there is $99 \%$ probability of being within three standard deviations of the 


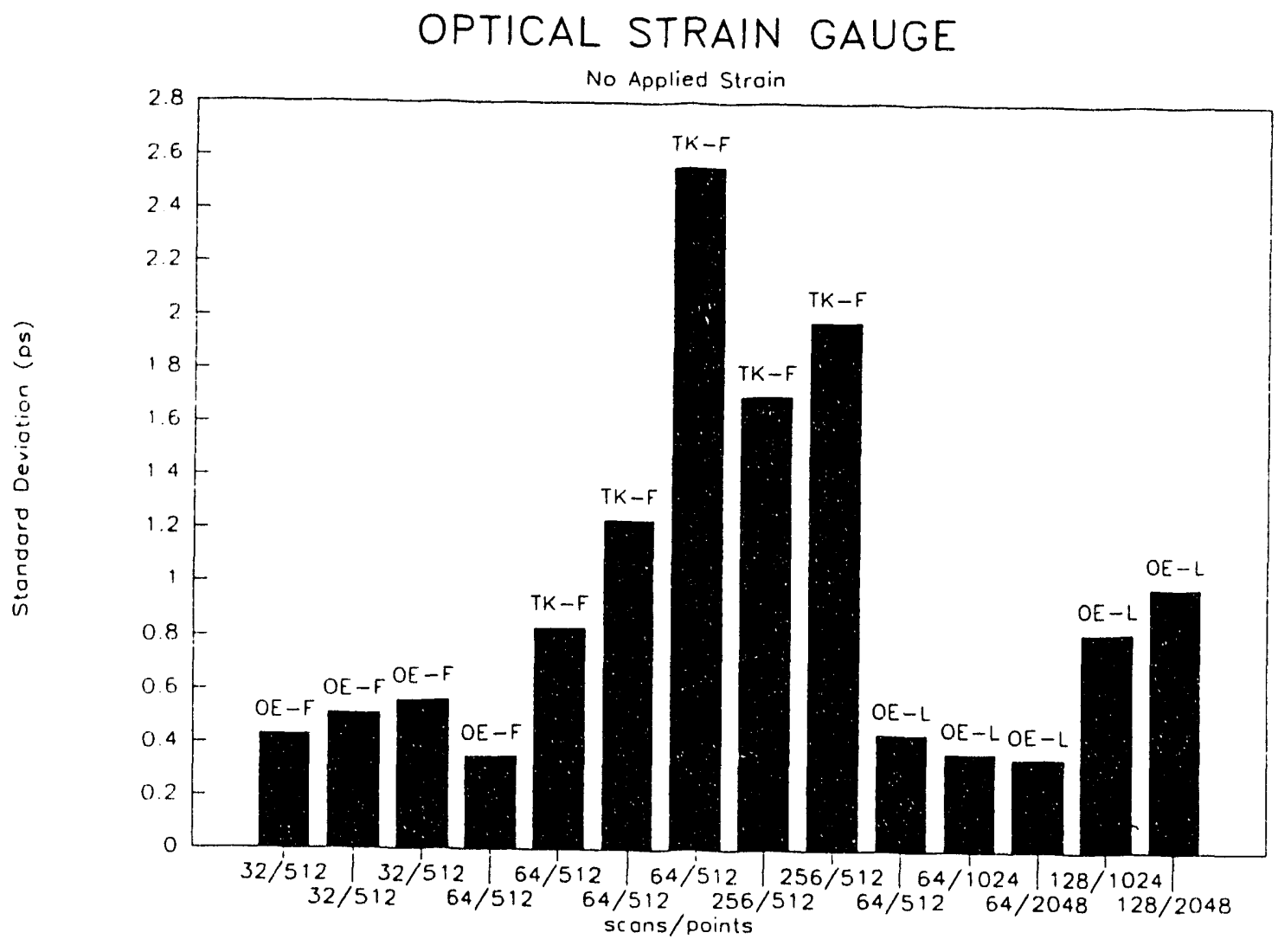

Fig. 50. Standard deviation for several layouts.

mean, then, for these data, the error in the data has $99 \%$ probability of being within $\pm(0.69+3 \times 0.55)= \pm 2.34 \mathrm{ps}$, significantly worse than the \pm 0.5 -ps uncertainty, of which the unit is capable under better circumstances. 


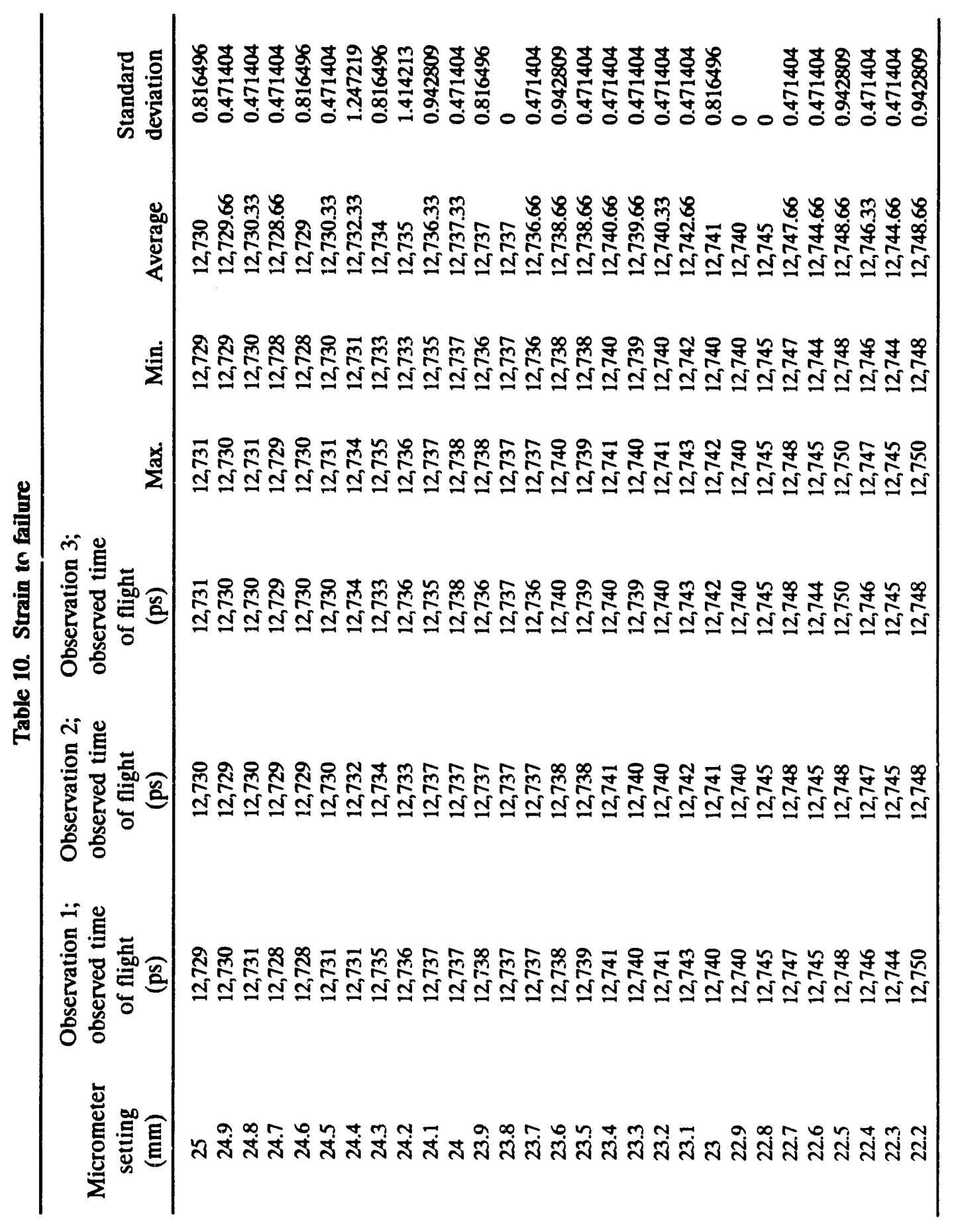




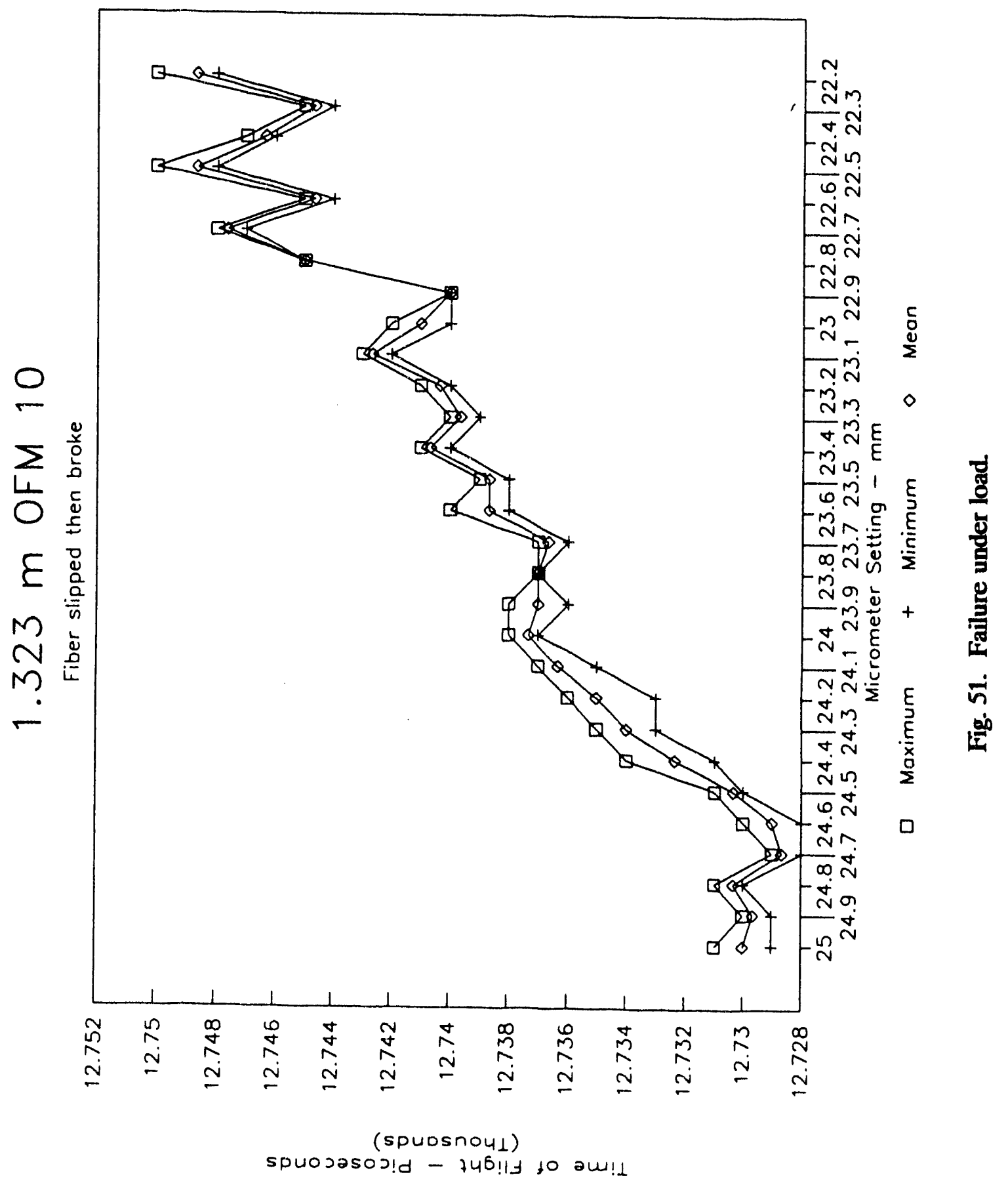


Table 11. Time of flight measured with OFM 10 fiber monitor

(Fiber-optic strain gauge;

bare fiber epoxied to mountings;

two observations made at each setting of micrometer;

epoxy failed at setting of $26 \mathrm{~mm}$;

baseline time of flight with no applied

strain was between 12,409 and $12,411 \mathrm{ps}$ )

\begin{tabular}{|c|c|c|c|c|}
\hline $\begin{array}{c}\text { Micrometer } \\
\text { setting } \\
(\mathrm{mm})\end{array}$ & $\begin{array}{c}\text { Run } 1 \\
\text { observed time } \\
\text { of flight } \\
\text { (ps) }\end{array}$ & $\begin{array}{c}\text { Run } 2 \\
\text { observed time } \\
\text { of flight } \\
\text { (ps) }\end{array}$ & $\begin{array}{c}\text { Run } 1 \\
\text { least squares } \\
\text { time of flight } \\
\text { (ps) }\end{array}$ & $\begin{array}{c}\text { Run } 2 \\
\text { least squares } \\
\text { time of flight } \\
\text { (ps) }\end{array}$ \\
\hline 39 & 12,413 & 12,412 & $12,412.860$ & $12,412.11$ \\
\hline 38.9 & 12,415 & 12,413 & $12,413.617$ & $12,412.89$ \\
\hline 38.8 & 12,413 & 12,414 & $12,414.375$ & $12,413.66$ \\
\hline 38.7 & 12,415 & 12,415 & $12,415.132$ & $12,414.43$ \\
\hline 38.6 & 12,416 & 12,416 & $12,415.889$ & $12,415.21$ \\
\hline 38.5 & 12,417 & 12,416 & $12,416.647$ & $12,415.98$ \\
\hline 38.4 & 12,417 & 12,417 & $12,417.404$ & $12,416.75$ \\
\hline 38.3 & 12,417 & 12,417 & $12,418.162$ & $12,417.53$ \\
\hline 38.2 & 12,418 & 12,418 & $12,418.919$ & $12,418.30$ \\
\hline 38.1 & 12,421 & 12,419 & $12,419.676$ & $12,419.07$ \\
\hline 38 & 12,421 & 12,420 & $12,420.434$ & $12,419.84$ \\
\hline 37.9 & 12,423 & 12,421 & $12,421.191$ & $12,420.62$ \\
\hline 37.8 & 12,421 & 12,422 & $12,421.949$ & $12,421.39$ \\
\hline 37.7 & 12,421 & 12,421 & $12,422.706$ & $12,422.16$ \\
\hline 37.5 & 12,424 & 12,424 & $12,424.221$ & $12,423.71$ \\
\hline 37.3 & 12,426 & 12,426 & $12,425.736$ & $12,425.26$ \\
\hline 37.1 & 12,426 & 12,426 & $12,427.251$ & $12,426.80$ \\
\hline 36.9 & 12,429 & 12,428 & $12,428.766$ & $12,428.35$ \\
\hline 36.7 & 12,430 & 12,430 & $12,430.280$ & $12,429.90$ \\
\hline 36.5 & 12,433 & 12,431 & $12,431.795$ & $12,431.44$ \\
\hline 36.3 & 12,433 & 12,432 & $12,433.310$ & $12,432.99$ \\
\hline 36 & 12,435 & 12,434 & $12,435.582$ & $12,435.31$ \\
\hline 35.6 & 12,438 & 12,437 & $12,438.612$ & $12,438.40$ \\
\hline 35.2 & 12,443 & 12,442 & $12,441.642$ & $12,441.50$ \\
\hline 34.8 & 12,445 & 12,447 & $12,444.672$ & $12,444.59$ \\
\hline 34.4 & 12,448 & 12,447 & $12,447.701$ & $12,447.68$ \\
\hline 34 & 12,451 & 12,450 & $12,450.731$ & $12,450.78$ \\
\hline 3.3 .5 & 12,455 & 12,455 & $12,454.518$ & $12,454.64$ \\
\hline 33 & 12,459 & 12,459 & $12,458.305$ & $12,4.58 .51$ \\
\hline 32.5 & 12,462 & 12,464 & $12,462.092$ & $12,462.37$ \\
\hline 32 & 12,466 & 12,467 & $12,465.879$ & $12,466.24$ \\
\hline 31.5 & 12,469 & 12,468 & $12,469.667$ & $12,470.11$ \\
\hline 31 & 12,474 & 12,474 & $12,473.454$ & $12,473.97$ \\
\hline 30 & 12,482 & 12,484 & $12,481.028$ & $12,481.71$ \\
\hline 29 & 12,488 & 12,489 & $12,488.602$ & $12,489.44$ \\
\hline 28 & 12,495 & 12,496 & $12,496.176$ & $12,497.17$ \\
\hline
\end{tabular}




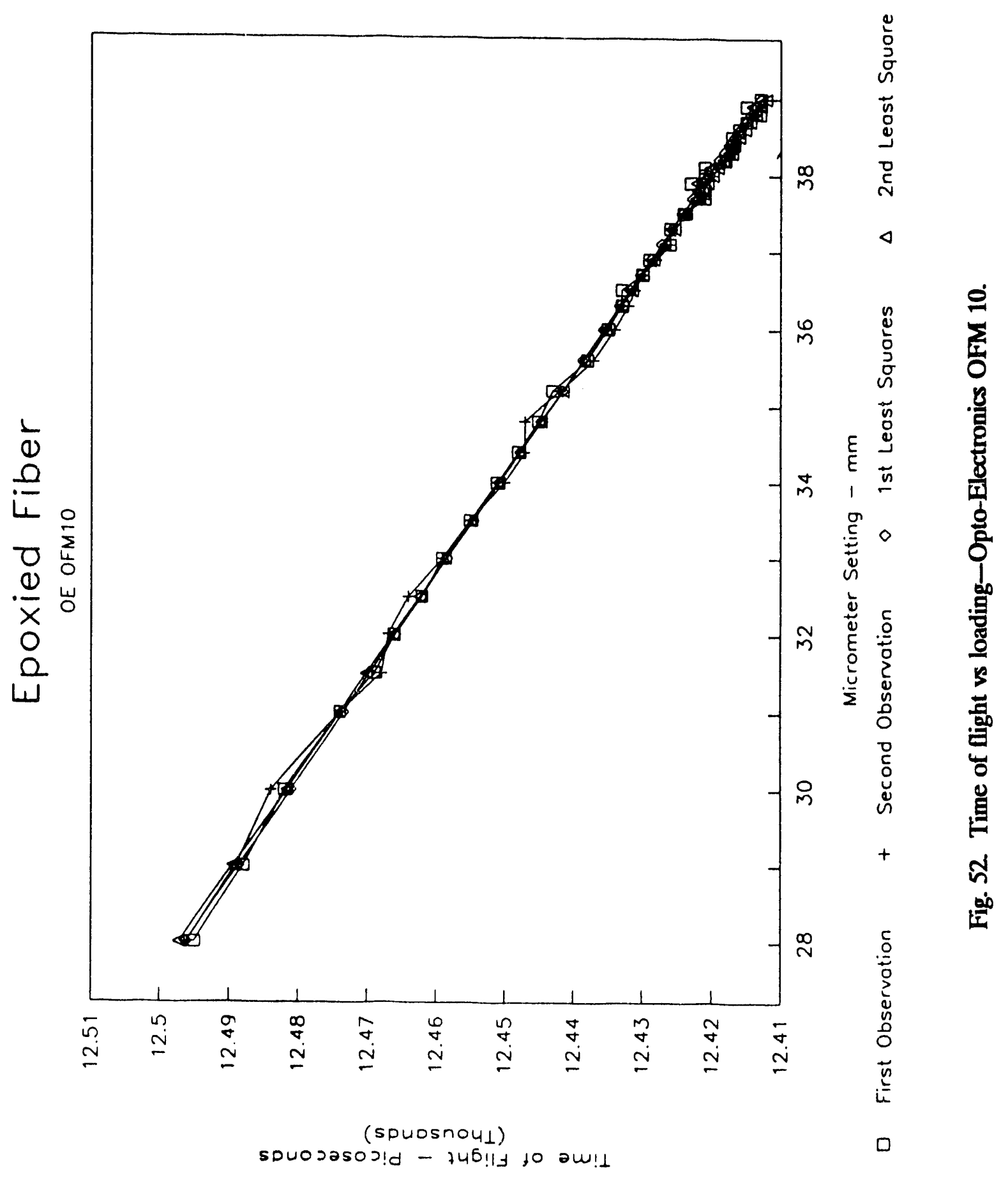




\section{OBSERVATION VS THEORY}

From the section on theoretical basis, the following equations are relevant:

$$
\frac{d N}{d L}=-\left(\frac{N^{3}}{2 L}\right)\left[\rho_{12}-\nu\left(\rho_{11}+\rho_{12}\right)\right] \text {. }
$$

In this equation, $\rho_{i j}$ are strain optic tensor components, $\nu$ is Poisson's ratio, $L$ is the gauge length, and $N$ is the index of refraction. follows:

The fiber photoelastic coefficient is $a$ [Zimmermann 1990a]. Zimmermann defines $a$ as

$$
a=\frac{L}{N} \frac{d N}{d L}
$$

Substituting the first equation into the second leads to:

$$
a=-\left(\frac{N^{2}}{2}\right)\left[\rho_{12}-\nu\left(\rho_{11}+\rho_{12}\right)\right] \text {. }
$$

The expression for strain is as follows:

$$
\epsilon=\frac{\Delta t_{1}-\Delta t_{0}}{K L \frac{N}{c}}\left[\frac{1}{1+a}\right],
$$

where

$\epsilon=$ strain,

$\Delta t_{1}-\Delta t_{0}=$ time between OTDR peaks displayed on scope,

$L=$ gauge length,

$c=$ speed of light in free space,

$N=$ fiber index of refraction (sample can be measured),

$a=$ fiber photoelastic coefficient (sample can be measured),

$K=$ the number of times the pulse passes through the gauge,

$$
K t_{f}=\Delta t_{1}-\Delta t_{0}
$$

$t_{\mathrm{f}}=$ time of flight of one pass through the gauge.

Substituting this expression into the strain equation leads to an expression for strain vs the time of flight of a single pass through the gauge:

$$
\epsilon=\frac{t_{f}}{L \frac{N}{c}}\left[\frac{1}{1+a}\right] .
$$


For a plot of time of flight of one pass through the gauge vs applied strain, the slope (assuming strain as a pure number and consistent units for other quantities) is given by the following:

$$
m=\frac{t_{f}}{\epsilon}=\frac{N L(1+a)}{c}
$$

If $t_{f}$ is in picoseconds and $L$ is in meters, then $c$ is $3 \times 10^{-4} \mathrm{~m} / \mathrm{ps}$. To find the slope of $t_{f}$ vs $\epsilon$, for $\epsilon$ in microstrains, as in the observed data tabulated in Tables 3 to 7, a correction factor of $10^{-6}$ is required.

$$
\begin{aligned}
& m=\frac{d t_{f}}{d \epsilon}=\frac{\Delta t_{f}}{\Delta \epsilon}=\frac{N L(1+a)}{c} \times 10^{-6} \\
& a=-\left(\frac{N^{2}}{2}\right)\left[\rho_{12}-\nu\left(\rho_{11}+\rho_{12}\right)\right],
\end{aligned}
$$

where

$m=$ the slope of the time vs strain relationship,

$\epsilon=$ strain (microstrains),

$t_{f}=$ time of flight of one pass through the gauge (ps),

$L=$ gauge length $(\mathrm{m})$,

$c=$ speed of light in free space $\left(3 \times 10^{-4} \mathrm{~m} / \mathrm{ps}\right)$,

$N=$ fiber index of refraction (pure number),

$a=$ fiber photoelastic coefficient (pure number),

$\rho_{i j}=$ fiber strain optic tensor components (pure number),

$\nu=$ Poisson's ratio of fiber (pure number).

It is the computation, from fiber parameters, of $m$, the slope of the time-vs-strain relationship, that is the theoretical prediction that can be compared with experimental observations.

Fused silica has the following properties [Brininstool 1987]:

$$
\begin{gathered}
N=1.478, \\
\nu=0.17, \\
\rho_{11}=0.121, \\
\rho_{12}=0.27 .
\end{gathered}
$$

The gauge length $L$ was measured as $1.323 \mathrm{~m}$ in experiments 1 to 3 and $1.2975 \mathrm{~m}$ in experiments 4 and 5 . 
Applying these parameters to the formulas leads to theoretical slope values of 0.005069 for experiments 1 to 3 and 0.004971 for experiments 4 and 5. The observed slope is the slope of the least squares line for each pass of each experiment. These slopes were computed to generate the least squares data in Tables 3 to 7. Percent error is computed by assuming that the theoretical prediction "s the "correct" value. Table 12 indicates that there is good agreement between theory and observation.

Ta.ole 12 Observation compared to theory

(Comparison of theoretical and observed values of slope in the time-of-flight vs strain relationship)

\begin{tabular}{cccccc}
\hline Experiment & Pass & $L$ & $\begin{array}{c}\text { Theoretical } \\
\text { slope }\end{array}$ & $\begin{array}{c}\text { Observed } \\
\text { slope }\end{array}$ & $\begin{array}{c}\text { Percent } \\
\text { error }\end{array}$ \\
\hline 1 & 1 & 1.323 & 0.005069 & 0.005133 & 1.26 \\
1 & 2 & 1.323 & 0.005069 & 0.005115 & 0.91 \\
1 & 3 & 1.323 & 0.005069 & 0.004761 & 6.08 \\
1 & 4 & 1.323 & 0.005069 & 0.004897 & 3.39 \\
2 & 1 & 1.323 & 0.005069 & 0.005233 & 3.24 \\
2 & 2 & 1.323 & 0.005069 & 0.005442 & 7.36 \\
2 & 3 & 1.323 & 0.005069 & 0.005052 & 0.34 \\
2 & 4 & 1.323 & 0.005069 & 0.005242 & 3.41 \\
3 & 1 & 1.323 & 0.005069 & 0.005697 & 12.39 \\
3 & 2 & 1.323 & 0.005069 & 0.005488 & 8.27 \\
3 & 3 & 1.323 & 0.005069 & 0.005324 & 5.03 \\
3 & 4 & 1.323 & 0.005069 & 0.005352 & 5.58 \\
3 & 5 & 1.323 & 0.005069 & 0.005379 & 6.12 \\
3 & 6 & 1.323 & 0.005069 & 0.005497 & 8.44 \\
4 & 1 & 1.2975 & 0.004971 & 0.004678 & 5.90 \\
4 & 2 & 1.2975 & 0.004971 & 0.004634 & 6.79 \\
5 & 1 & 1.2975 & 0.004971 & 0.004977 & 0.11 \\
5 & 2 & 1.2975 & 0.004971 & 0.004914 & 1.15 \\
5 & 3 & 1.2975 & 0.004971 & 0.004781 & 3.83 \\
5 & 4 & 1.2975 & 0.004971 & 0.004856 & 2.32 \\
5 & 5 & 1.2975 & 0.004971 & 0.004852 & 2.40 \\
5 & 6 & 1.2975 & 0.004971 & 0.004772 & 4.01 \\
\hline
\end{tabular}




\section{AREAS OF FURTHER EXPLORATION}

The major conclusion of this experimentation is that submillimeter OTDR is a practical way of measuring strain in optical fiber. In the Fresnel setup, strain sensitivity of 400 microstrains was observed. In the loop setup, strain sensitivity of 200 microstrains was observed. Both setups used a single-mode fiber with a 1-m gauge length and a 50-50 directional coupler. The predominant limit to performance was \pm 2 -ps time jitter. A secondary limit on performance was the $6.4-\mathrm{gHz}$ bandwidth of the detection electronics.

Four areas are of further interest. First, having found that the performance of the OTDR setup based on general-purpose instruments was limited by time jitter and receiving bandwidth, it is desirable to find the limits of performance of an OTDR system optimized for low trigger jitter and high bandwidth. Second, having demonstrated principle with the loop strain gauge, it is desirable to find the practical limits of its perforinance. Third, it is desirable to obtain time-of-flight vs load data for a fiber embedded in composite. Fourth, if military sponsorship of further development is desired, it will probably be necessary to demonstrate the capability of making simultaneous temperature measurements and temperaturecompensated strain measurements over the range of $-30^{\circ} \mathrm{C}$ to $100^{\circ} \mathrm{C}$, but not necessarily with embedded fibers.

To test the capability of a low-jitter, high-bandwidth OTDR system, an integrated submillimeter OTDR system will be rented from Opto-Electronics. Time-of-flight vs strain measurements will be made for both the Fresnel and looping strain gauges using the 50-50 directional coupler. The object of this experiment will be to compare the repeatability of these observations with those taken with the Tektronix setup. It is expected that the uncertainty in time between peaks should improve by a factor of 4 , from \pm 2 ps with the old setup to \pm 0.5 ps with the new setup.

To test the limits of the looping strain gauge, the loop experiments will be rerun with the Opto-Electronics OTDR and a 90-10 tap-off coupler. Of particular interest is the question of whether or not our single-mode system will outperform the multimode system reported by Zimmermann. It is expected that the observed number of transits through the gauge fiber should increase by at least a factor of 5 , from 4 with the old setup to 20 with the new setup. By using the Opto-Electronics OTDR and the 90-10 coupler, the sensitivity of the loop strain gauge should improve by a factor of 20 , from 200 to 10 microstrains, with a $1-\mathrm{m}$ gauge length.

To test the capability of the system to measure strain in a composite, a loop strain gauge system similar to that shown in Fig. 53 will be fabricated. In this scheme, two single-mode fibers will be embedded in a sample during the compositing process. The two fibers will be parallel and relatively close to each other. They will indicate the average longitudinal strain in their area of the sample. The sample will be on the order of a foot long, resulting in an effective gauge length of about $0.5 \mathrm{~m}$. The method of applying load to the sample has not yet been decided.

The fourth experiment will be the simultaneous measurement of strain and temperature in a single fiber. Several strain-independent techniques of temperature measurement are being explored. The most promising of these will be applied simultaneously with OTDR to obtain a temperature reading and a time-of-flight reading that can be combined to determine the temperature-compensated strain. In the effort to obtain sponsorship for follow-on research, it is probably more desirable to demonstrate such a system over a temperature range of $-30^{\circ} \mathrm{C}$ to $100^{\circ} \mathrm{C}$ in an unembedded environment than to demonstrate an embedded system over a narrower temperature range. 


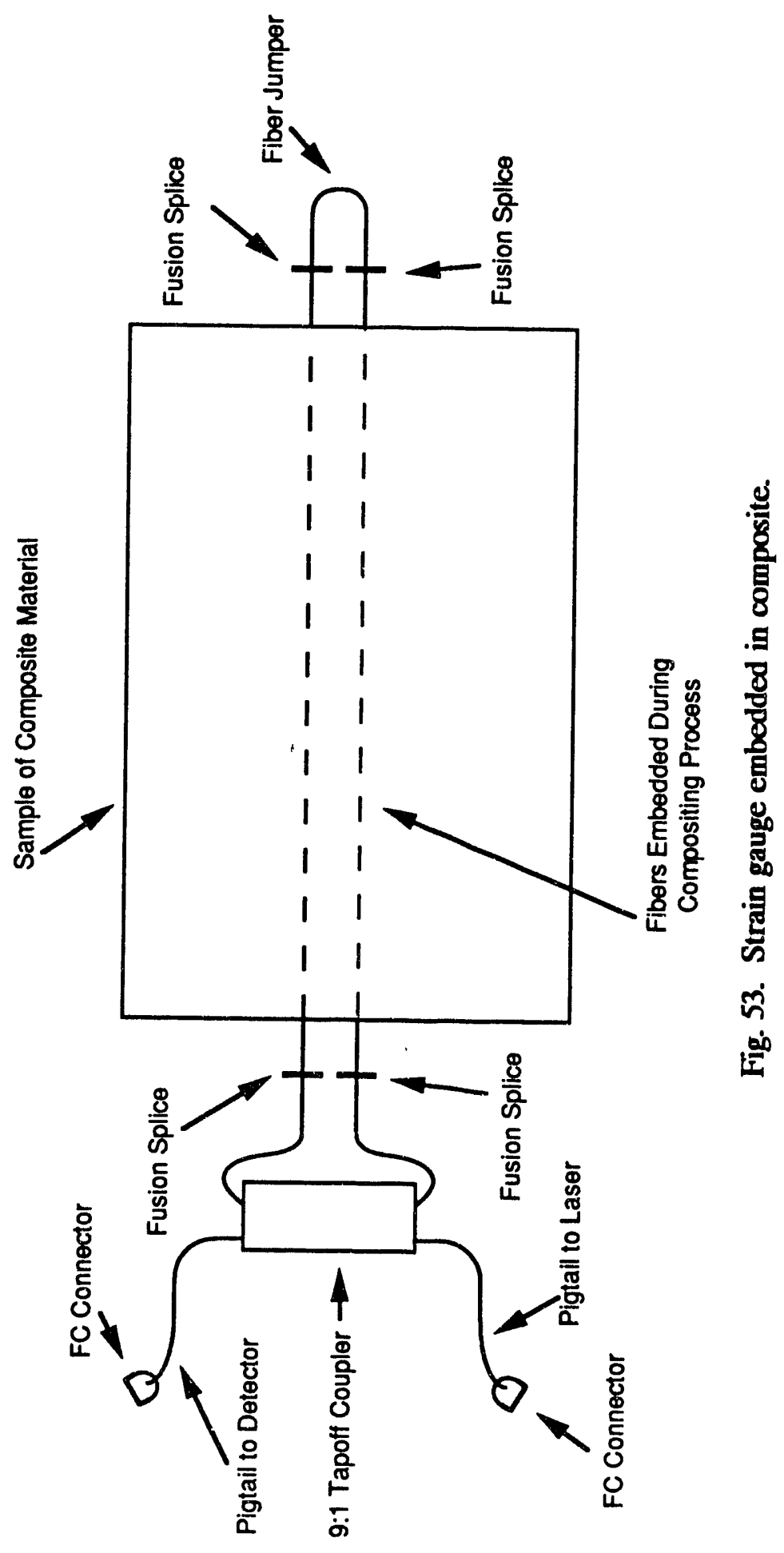




\section{REFERENCES}

[Brininstool 1987]

[Dunphy 1982]

[Garside 1988]

[Jackson 1984]

[Kercel 1992]

[Marvin 1984]

[Murphy 1990]

[Udd 1991]

[Waite 1988]

[Zimmermann 1990a]

[Zimmermann 1990b]
Brininstool, M. R. 1987. "Measuring Longitudinal Strain in Optical Fibers," Optical Engineering 26(11), 1112-1119.

Dunphy, J. R., Meltz, G., and Snitzer, E. 1982. "A Fiber Optic Strain Gage Based on Crosstalk," Engineering Applications of Optical Measurements, pp. 77-78.

Garside, B. K. 1988. "Ultrahigh Resolution Optical Time Domain Reflectometry," SPIE Volume 954 Optical Testing and Metrology II, pp. 670-675.

Jackson, B. S. 1984. Optical Time Domain Reflectometry As a Nondestructive Evaluation Technique for Composite Materials, Master's Thesis, Virginia Polytechnic Institute and State University, Blacksburg.

Kercel, S. W., and Muhs, J. D. 1992. "Repeatable Sensitivity of OTDR-Based Strain Measurement," SPIE Volume 1757, Ultrahigh- and High-Speed Photography, Videography, and Photonics '92, p. 60.

Marvin, D. C., and Ives, N. A. 1984. "Wide-Range Fiber-Optic Strain Sensor," Applied Optics 23(23), 4212-4217.

Murphy, K. A., Miller, M. S., Vengsarkar, A. M., and Claus, R. O. 1990. "Elliptical-Core Two-Mode Optical-Fiber Sensor Implementation Methods," Journal of Lightwave Technology 8(11), 1688-1696.

Udd, Eric. 1991. Fiber Optic Sensors, John Wiley and Sons, New York, ISBN 0-471-83007-0.

Waite, S. R., Tatum, R. M., and Jackson, D. A. 1988. "Use of Optical Fibre for Damage and Strain Detection in Composite Materials," Composites 19(6), 435-442.

Zimmermann, B. D., Claus, R. O., Kapp, D. A., and Murphy, K. A. 1990. "Fiber-Optic Sensors Using High-Resolution Optical Time Domain Instrumentation Systems," Journal of Lightwave Technology 8(9), 1273-1277.

Zimmermann, B. D., Thomas, D., and Claus, R. O. 1990. "Recent Progress in High Resolution Optical Fiber Time Domain Methods and Its Impact on Dynamic Environment Sensing Applications," SPIE Volume 1370, Fiber Optic Structures and Smart Skins III, pp. 107-204. 
ORNL/TM-12383

\section{INTERNAL DISTRIBUTION}

1-5. S. W. Kercel

6. H. R. Brashear

7. B. G. Eads

8. D. N. Fry

9. D. W. McDonald

10. D. R. Miller

11. R. E. Uhrig

12. J. O. Stiegler

13. J. D. Muhs
14. G. N. Miller

15. D. F. Craig

16-17. Central Research Library

18. Y-12 Technical Reference Section

19-20. Laboratory Records

21. Laboratory Records-Record Copy

22. ORNL Patent Section

23. I\&C Division Publications Office

\section{EXTERNAL DISTRIBUTION}

24. Assistant Manager for Energy Research and Development, DOE-ORO, P.O. Box 2001, Oak Ridge, TN 37831-8600

25. B. Chexal, Electric Power Research Institute, 3412 Hillview Avenue, Palo Alto, CA 94303

26. V. Radeka, Brookhaven National Laboratory, Instrumentation Division, 535-B, Upton, NY 11973

27. M. M. Sevik, Carderock Division, Naval Surface Warfare Center, Code 1900, Bethesda, MD 20084-5000

28. R. M. Taylor, Leeds and Northup, Sunneytown Pike, North Wales, PA 19454

29-30. Office of Scientific and Technical Information, U.S. Department of Energy, P.O. Box 62, Oak Ridge, TN 37831 

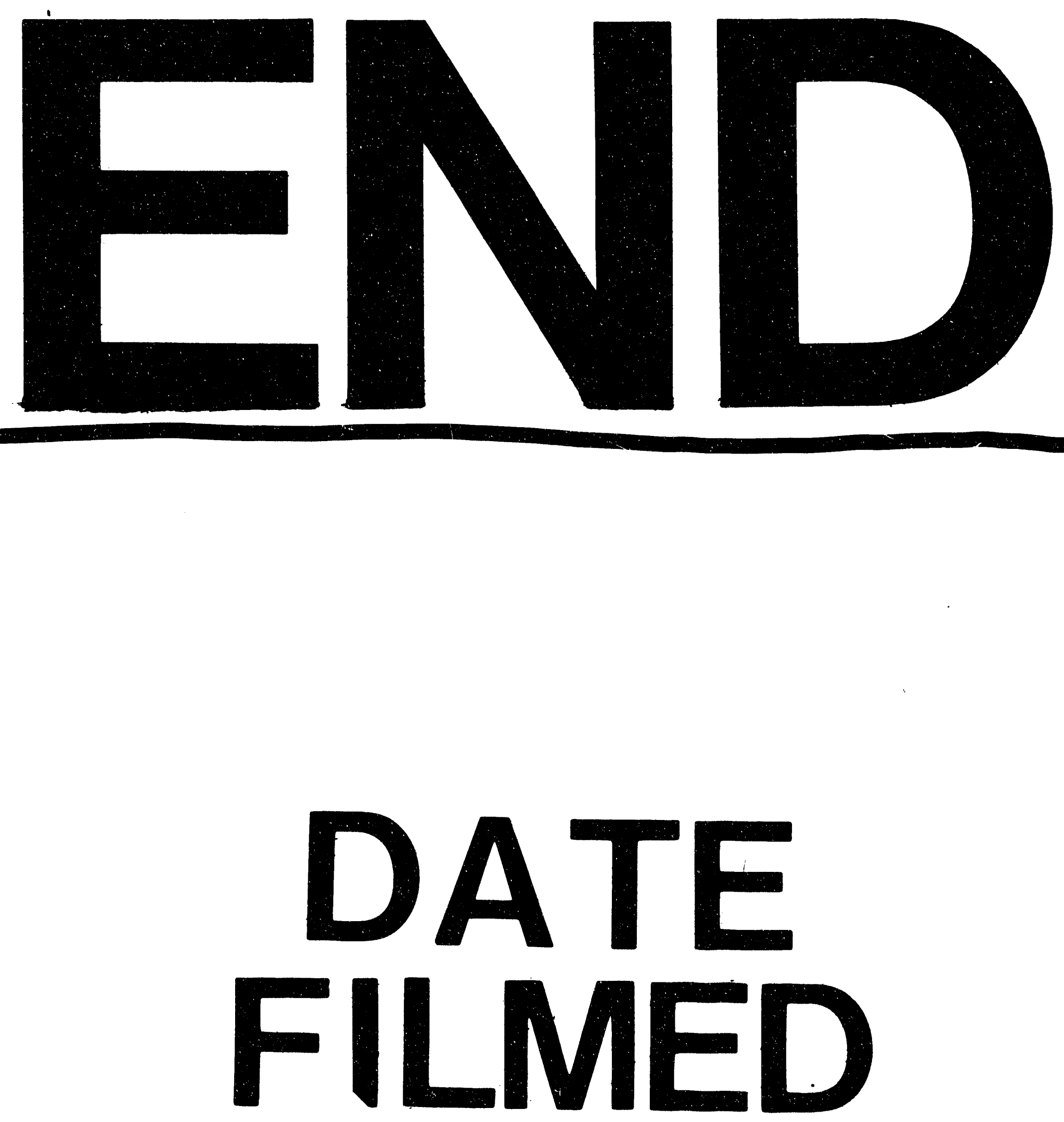

1

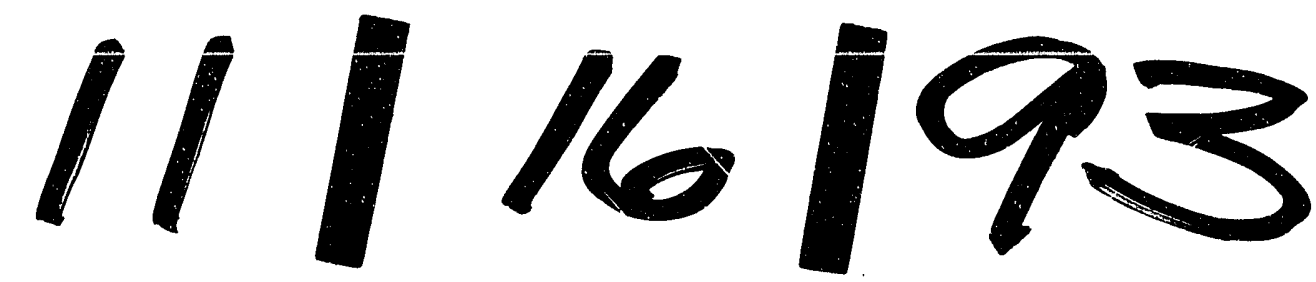


